Florida International University

FIU Digital Commons

\title{
Transnational Sex-Positive Play Parties: The Sexual Politics of Care for Community-Making at a Kinky Salon
}

\author{
Christina Bazzaroni \\ Florida International University, cbazz001@fiu.edu
}

Follow this and additional works at: https://digitalcommons.fiu.edu/etd

Part of the Gender and Sexuality Commons, Human Geography Commons, Leisure Studies Commons, Other Feminist, Gender, and Sexuality Studies Commons, Other Geography Commons, Politics and Social Change Commons, Social and Cultural Anthropology Commons, and the Women's Studies Commons

\section{Recommended Citation}

Bazzaroni, Christina, "Transnational Sex-Positive Play Parties: The Sexual Politics of Care for CommunityMaking at a Kinky Salon" (2019). FIU Electronic Theses and Dissertations. 4050.

https://digitalcommons.fiu.edu/etd/4050

This work is brought to you for free and open access by the University Graduate School at FIU Digital Commons. It has been accepted for inclusion in FIU Electronic Theses and Dissertations by an authorized administrator of FIU Digital Commons. For more information, please contact dcc@fiu.edu. 


\section{FLORIDA INTERNATIONAL UNIVERSITY \\ Miami, Florida}

TRANSNATIONAL SEX-POSITIVE PLAY PARTIES:

THE SEXUAL POLITICS OF CARE FOR COMMUNITY-MAKING

AT A KINKY SALON

A dissertation submitted in partial fulfillment of the

requirements for the degree of

DOCTOR OF PHILOSOPHY

in

GLOBAL AND SOCIOCULTURAL STUDIES

by

Christina Nicol Bazzaroni

2019 
To: Dean John F. Stack, Jr.

Steven J. Green School of International \& Public Affairs

This dissertation, written by Christina Nicol Bazzaroni, and entitled Transnational SexPositive Play Parties: The Sexual Politics of Care for Community-Making at a Kinky Salon, having been approved in respect to style and intellectual content, is referred to you for judgment.

We have read this dissertation and recommend that it be approved.

$\begin{array}{r}\text { Andrea Queeley } \\ \hline \text { Laurie Shrage } \\ \hline \text { Benjamin Smith } \\ \hline \text { Caroline Faria, Co-major Professor } \\ \hline \text { Jean Muteba Rahier, Co-major Professor }\end{array}$

Date of Defense: March 29, 2019

The dissertation of Christina Nicol Bazzaroni is approved.

Dean John F. Stack, Jr. Steven J. Green School of International \& Public Affairs

Andrés G. Gil Vice President for Research and Economic Development and Dean of the University Graduate School

Florida International University, 2019 
C Copyright 2019 by Christina Nicol Bazzaroni

All rights reserved. 


\section{DEDICATION}

To my parents, thank you. 


\section{ACKNOWLEDGMENTS}

I am honored and humbled to extend my gratitude to all those who supported me in obtaining my graduate degree. First, I thank the Florida International University, University Graduate School, for awarding me the Dissertation Year Fellowship. This fellowship helped me to write this manuscript and graduate, supported, in a timely manner. Next, I thank my chair, Professor Jean Muteba Rahier. Dr. Rahier has been instrumental in every step of my academic success, providing me valuable personal and professional guidance and support. Thank you also to my co-chair, Professor Caroline Faria. Dr. Faria has shaped my understandings of what feminist geographies may be, exciting me to go in the direction I have. Both Dr. Faria and Dr. Rahier have been generous and supportive allies throughout this process. Without their encouragement this project would not have been completed, I am very grateful to you both. I also want to thank my dissertation committee: Professor Ben Smith, Professor Andrea Queeley, and Professor Laurie Shrage. Your commitment to my completion is deeply appreciated. I also want to thank fellow colleagues I have met and become enduring friends with while on my academic journey, your support and encouragement has mattered greatly. And finally, I extend immense gratitude to all the people that made this work possible, your warmth, trust and enthusiasm has been humbling and enriching. Thank you all for all you have taught me on this journey. 


\author{
ABSTRACT OF THE DISSERTATION \\ TRANSNATIONAL SEX-POSITIVE PLAY PARTIES: \\ THE SEXUAL POLITICS OF CARE FOR COMMUNITY-MAKING \\ AT A KINKY SALON \\ by \\ Christina Nicol Bazzaroni \\ Florida International University, 2019 \\ Miami, Florida \\ Professor Jean Muteba Rahier, Co-Major Professor \\ Professor Caroline Faria, Co-Major Professor
}

To date, feminist geographers and geographers of sexualities have yet to fully interrogate post sexual revolution society. In this dissertation I examine the politics of sex-positive play parties, through the case study of Kinky Salon (KS) - a global organization that claims to catalyze a contemporary sex culture revolution. This project expands on previous feminist geography and geographies of sexualities scholarship centering queer, kinky sex, demonstrating that non-normative sexual practices are informed by and contribute to sexual revolution legacies. I extend feminist geographies' theorizing of affect and emotion to show how sexual intimacies are care-work, with the emotional power to bring about relation-building and sexual understanding. In doing so, play-based sex-positive politics are highlighted as a framework to promote community, and resistance against norms that constrain sexually deviant bodies.

This project highlights the complexities of sex-based efforts at social change, which I show continue to reflect inequalities in society even as they seek to transform it. I 
begin by asking: What is so political about playful sex? Answering this guiding question required a multi-sited, mixed methodological, ethnographic approach, to undertake a feminist geographical exploration of embodied sexuality, play, and care as activism. It took two years of field research to gain trust among members of a sex-positive community. I conducted fifty-three semi-structured interviews, and countless hours of informal conversations, proving crucial to my overall understanding of sex-positive culture. Time spent in the field was enriched by observant-participation as a volunteer, culminating in a transnational tour of a global community.

The data collected underscores the political contestations of inclusivity ethics and the transnational spread of sex culture aimed at changing discourses about deviant sex. I show that play is constructed as transformative for community members who adopt activist non-normative care practices that require new theorizing of sexual subjectivity. This project brings together geographies of sexualities and feminist geographies to move them forward. By revealing how affect, emotion, and intimacy, are co-constituted, I suggest that there is an opportunity to more fully explore what care ethics has to offer sexuality studies. 


\section{TABLE OF CONTENTS}

I. INTRODUCTION \& LITERATURE REVIEW: Sex parties as spaces for political play: Community-making and sexual revolution through Kinky Salon ............. Introduction

Intellectual setting: Situating the challenges and potentials of sex-positive culture .................................................................

Contextualizing sexual revolutions and their legacies......................................

Geographies of sexuality: Constructing sexual dissidents and cultural diasporas.....

Playing with a politics of care: Toward an affective, sex culture

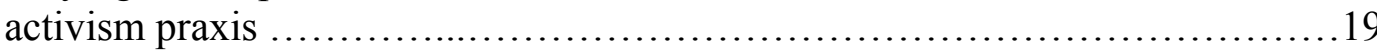

Research Objectives ..................................................25

What is Kinky Salon and how does it work to influence sex culture? ...........27

Chapter summaries .................................................... 37

Endnotes ............................................................40

II. METHODOLOGY \& METHODS: The embodied researcher in a sexualized field....44 Introduction: A project in the making, journeying to the topic .................44 Feminist methods in geography: Coming to terms with intangible geographies ..45 Sexuality research and self-care: Understanding fieldwork as a site of deep reflexivity ........................................................... 48

Creating boundaries: Negotiating intimacy and emotional entanglements in the field ............................................................. 51

Managing the stigma and potential for shame in doing sexuality research .......57

Feminist methods in action ...........................................57

Conclusion ..........................................................67

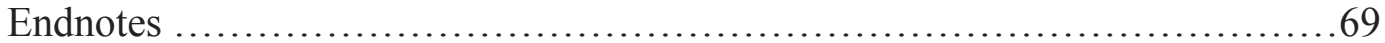

III. THE POLITICS OF PLAY: Negotiating power, connection, and transformation

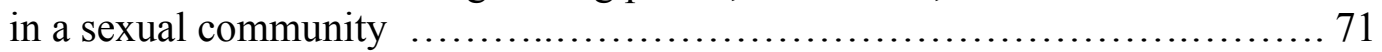

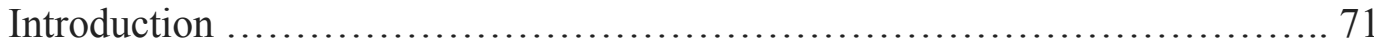

Intellectual setting and methods ........................................ 72

Establishing the playground rules: What is so political about playful sex? ......77

Negotiating power and connection: The policing of play ................... 90

The transformative potentials of sex-positive community and playful sex .......98

The marginalizing potentials of cultivating inclusive, sexual community ......107

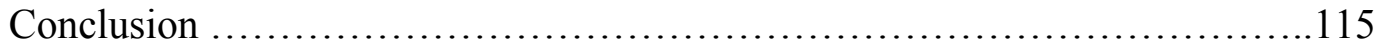

Endnotes ....................................................... 117

IV. CONSTRUCTING CARE IN SEX CULTURE: Reimagining revolutionary change one educated orgasm at a time? 


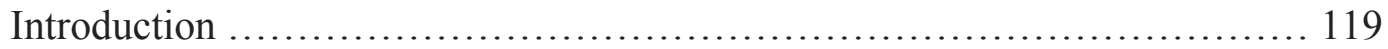

Intellectual setting and methods ...........................................120

Reimagining sexual revolution as softcore activism through

a politics of care ..................................................... 123

Enacting care ethics through radical acts of being, community-making and education ...........................................................

Failures and reconciliations of care: Realizing the impacts of radical acts

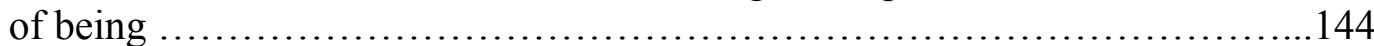

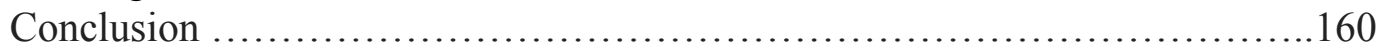

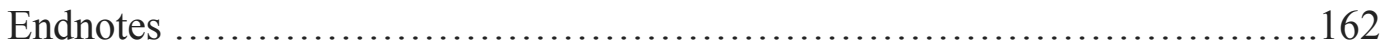

V. TRANSNATIONAL INTERROGATIONS OF SEX-POSITIVE CULTURE:

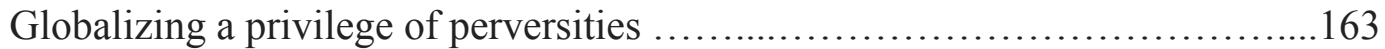

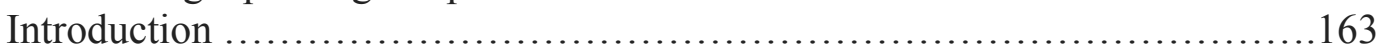

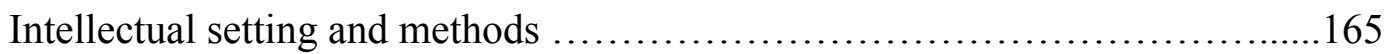

Transnational sexual community: Understanding the Kinky Salon Global

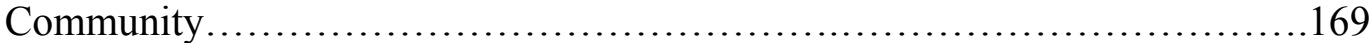

The globalization of sex culture activism ..................................175

What's care got to do with it? Transnational sex culture activism in place ......179 Challenges and disruptions to liberatory discourses of transnational

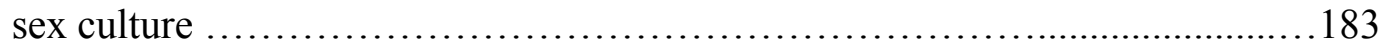

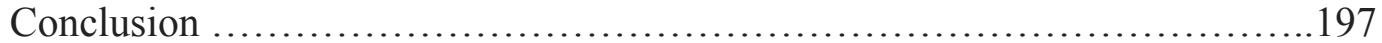

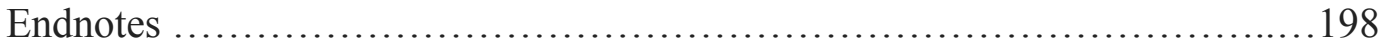

VI. CONCLUSION: Learning from failures: Deconstructing privilege to create a

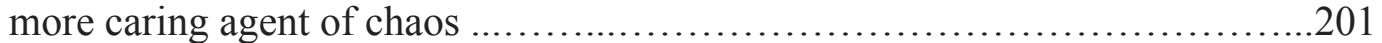

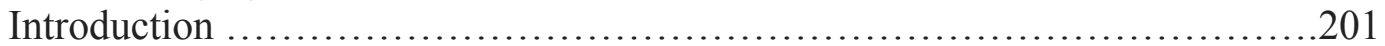

Reviewing the findings: Terrible, wonderful utopias .......................205

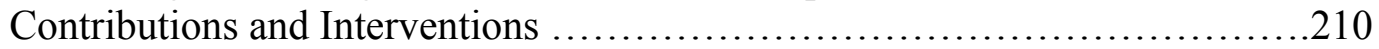

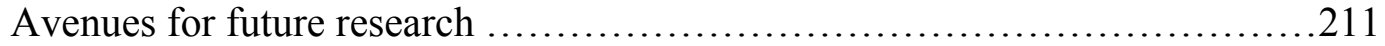

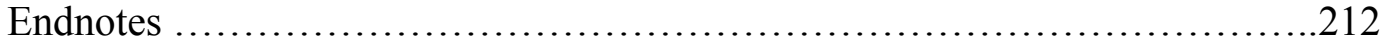

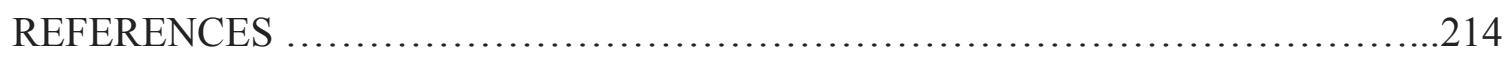

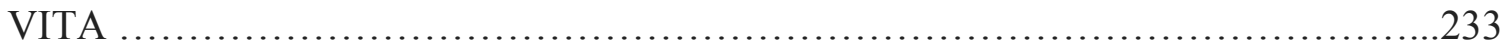




\section{ABBREVIATIONS AND ACRONYMS}

\begin{tabular}{ll} 
BDSM & Bondage Dominance Submission Masochism \\
BIPOC & Black Indigenous People of Color \\
BM & Burning Man \\
CNM & Consensual non-monogamy \\
KS & Kinky Salon \\
KSB & Kinky Salon Berlin \\
KSC & Kinky Salon Copenhagen \\
KSGC & Kinky Salon Global Community \\
KSL & Kinky Salon London \\
KSLA & Kinky Salon Los Angeles \\
KSNOLA & Kinky Salon New Orleans \\
KSNYC & Kinky Salon New York City \\
KSPDX & Kinky Salon Portland \\
KSSF & Kinky Salon San Francisco \\
KSS & Kinky Salon Seattle \\
LGBTQ & Lesbian Gay Bisexual Transgender Queer \\
PAL & Pervy Activity Liaison \\
POC & People of Color \\
SCR & Sex Culture Revolution \\
\hline
\end{tabular}




\section{INTRODUCTION \& LITERATURE REVIEW: \\ Sex parties as spaces for political play: \\ Community-making and sexual revolution through Kinky Salon}

\section{Introduction}

In general, discourses around sex, seeking sex, and safe spaces for queer and nonnormative sexuality ${ }^{1}$ are becoming more permissive. Adventurous attitudes toward sex are much less taboo, with a proliferation of sex toy shops, some of them identifying as queer, feminist, sex-positive, ${ }^{2}$ and female-centric (Hamer, 2014:18; see also ${ }^{3}$ ). These changes are being seen on a more widespread scale in the Global North (Altman, 2002; Binnie, 2004; Gorman-Murray, 2006). Sex party culture is one facet of this engagement with sexuality that is becoming popularized helped along by the ability to connect through new technologies. Though not necessarily mainstream, previously taboo or "perverse" sexual tastes are made more accessible to the curious and sexually adventurous in Global North, post-industrial societies. One such contribution of a socalled increasingly sexualized culture and globalization of sex enables possibilities for new identities, alliances, and resistance to emerge.

During my early research on sex culture, I watched John Cameron Mitchell's film, Shortbus (2006). ${ }^{4}$ I was struck by the depiction of public, queer $\operatorname{sex}^{5}$ that was politicized yet humanized in vividly creative ways. I understood this as a unique opportunity to explore the politics of a sex party in ways that I have not seen done, particularly in feminist or sexuality geographies. As I asked questions like, "Who is involved and how are these communities sustained?" I began to wonder if is it even important to explore something so taboo? My research drew me to Kinky Salon, an event and organization that seemed to embody these ideals. Although, I was challenged during 
early literature reviews to find forerunners that could help me situate an ethical, playful, sex party, where people seem to laud their personal transformation. So, I stepped up to the challenge of investigating. Having identified an absence both in the literature and the above referenced film, the data collected for this work challenges ideas of utopian sexual communities (Cooper, 2009) by pointing to the racial politics and reproduction of privilege that is undertheorized when it comes to liberatory sexuality.

In this dissertation I investigate a community organization that is built and sustained around sex-positive events referred to as a "play party." More loosely considered a sex party, Kinky Salon (KS) claims to provide an "alternative space to intimidating or impersonal sex clubs" (www.kinkysalon.com). The events and community are organized to represent a space of inclusivity, supporting personal growth, and sexual exploration; and happen regularly supported by a strong complimentary online and offline presence. Parties and events are completely run by volunteers, and taken together with its attendant community comprise what I loosely term an organization. The mission behind KS is to catalyze a contemporary "sex culture revolution." A "sex culture revolution" (SCR) in KS terms, seeks to normalize sex via alternative sexual practices (i.e. group/public sex, BDSM, kink, etc.) and alternormative sexuality ${ }^{7}$ (Cooper, 2009) and relationship structures (i.e. polyamory, consensual non-monogamy). This agenda seeks to weave sexuality into the fabric of daily life by attempting to "bring sex out of the shadows, ${ }^{8}$ " suggesting that what was once considered deviant can be reconceptualized. If it is successful, this is a powerful claim and one worth our attention. 


\section{Intellectual setting: Situating the challenges and potentials of sex-positive culture}

In this dissertation I center sexual play as both a political and a spatial act. To date, scholarly literatures both under-examine and undertheorize sex parties. There remains little work on sex parties or sex clubs. Most of the scholarship on the topic has focused on young, white, gay men. ${ }^{9}$ In particular, geographies of sexualities scholars have yet to engage with how sex parties may be spaces for sex culture activism to unfold. In response to this absence, my research is a critical intervention that underscores new ways of theorizing sexual politics, framing play with care as political. Thus, Kinky Salon makes a case for these arguments through its global reach, urging ideas of decommodification of sexuality and pleasure.

In this project I engage with feminist geographies' conceptualizations of affect, emotion, intimacy, and care. ${ }^{10} \mathrm{I}$ use this scholarship to build a case that sex culture activism can be understood in new ways. I engage with scholarship that critically situates the effects of community building, education, and resistance, from a transnational perspective. And I bring this scholarship into conversation with feminist, queer geographic work on sexuality. Therefore, this dissertation underscores how playful sex is constructed through care ethics as a means to disrupt globalizing, hegemonic sexuality discourses. I do so with the understanding that undertaking an analysis of the particularities and specificities of each location the events are held is beyond the scope of this project. The focus here is on care being the cement of the community as it is enacted through the expression of sexuality, rather than the depiction of actual sex acts in relation to the "globalizing" community, being the goal of this work. Accordingly, this project 
brings together these bodies of scholarship to investigate how sex-positive sex culture is developed and sustained through play parties and community-making.

\section{Contextualizing sexual revolutions and their legacies}

The case study I have focused on makes a strong statement of instigating a sex culture revolution. Yet, this begs the question how it is different from the sexual revolution of the 1960s and 1970s. To understand this I explore sexual political movements of the past to situate whether these claims are entirely new. Historians, women's studies, media studies, sociologists, critical race, sexuality studies, and feminist scholars ${ }^{11}$ are among many who have explored the sexual revolution of the 1960s and 1970s, their legacies, and contemporary ramifications. Within popular consciousness, we continue to understand the 1960s and 1970s as an entry point for the beginning of the sexual revolution. Social scientist and sexuality scholar Martin (1996), however, investigates the conceptual framework of sexual revolution, which he identifies as having a much older history beginning in the 1920s. The influences from the Bolshevik Revolution in Russia and the culture of Weimar Germany shaped changes in the United States in the 1920s and beyond (Martin, 1996). He argues that this framework has been appropriated and reworked for the changing times in the 1960s and 1970s, critiquing the use of revolutionary language in latter iterations.

However, the original theorist of the sexual revolution of the 1960s and 1970s, Marxist scholar Herbert Marcuse, was "concerned with the dominance of technological rationality and the contradictions of advanced industrial society... with a call to liberate sexuality from the strictures of bourgeois patriarchal morality" (Garlick, 2011:222). And even scholars of economics (Greenwood \& Guner, 2010) have pointed to that time as 
being pivotal in restructuring the power of female agency as it has been correlated with increased availability to contraceptives. Due to an "increase in mutually expected selfrestraint (mutual consent) in interactions" (Wouters, 1998:190), contraception is highlighted as a large part of what spurred new found bodily autonomy for women to make different family planning choices. Greenwood \& Guner (2010) claim that this contributed to the increased visibility regarding differential desires for sex. However, it has been argued by Allyn (2016) that in past movements lesbian feminists wished to reconceptualize sexual revolution to reconcile what was considered a "male conspiracy to make women more sexually available" (2016:245). Arguing that the pill was used as a way to pressure women into having sexual relations (Martin, 1996).

Toward the end of the sexual revolution, the "Golden Age" for free love among gay and lesbian communities represented new sexual freedoms with the establishment of bathhouses and sex clubs that provided safe spaces to have casual sex (Escoffier, 2008). Even more, the sexual revolution inspired new ways of conceiving relationships with open marriages, and key-swapping orgies. This was thought to be part of the new freedoms accepting of exploring sexuality, new relationships styles, and gender preferences (Escoffier, 2008). Care, love and sex in the counterculture movements of free love, women's empowerment, and the gay and lesbian movement, informed the sexual revolution, created out of co-opted and appropriated ideals through social movement spillover (Meyer \& Whittier, 1994; D'Emilio, 1983). The subject of this study claims that revolutionary newness can be found in reclaiming "love" into the matrix of care and alternative sexual relationships. But, at first glance, this doesn't appear to be different than the free love movement of the 1960s and 1970s when alternative relationships were 
being experimented with. For instance, the straight edge movement was a movement established in response to hippies and free love: "Whereas hippies viewed liberated sex as revolutionary... sXers saw abstinence from "promiscuous" sex as a powerful form of resistance" (Haenfler, 2004:419). Although the free love movement "rejected the responsibilities normally associated with sexual relationships: marriage, commitment, and children" (Haenfler, 2004:410), sex-positive community today tends to embrace responsibility, accountability and ownership as an overarching sentiment. The blending of the ideologies of these two previous movements seems to be at work within sexpositive sex culture presently. This suggests that claims for contemporary sexual revolution be reconceptualized as cyclical, rather than linear events which may at times recede and reform. These processes are more organic and responsive to the changing ways dissidents are constructed.

Complicating these histories further, sexuality scholar Volkmar Sigusch (1998), insists on a sexual revolution period that continued from the 1960s and 1970s into the 1980s and 1990s. He termed this period the "neosexual revolution." Sigusch argues that this neosexual revolution is marked by a deconstruction of old forms of sexuality and reformation around new relationship styles, and gender and sexuality preferences. However, as I have pointed out above, this was already taking place. He argued that, particularly in the face of a devastating AIDS crisis, new forms of intimacy were taking shape, which can be seen with the response to the AIDS crisis being one of managing a crisis of urgently needing care. Nevertheless, both Martin (1996) and Sigusch (1998) argue that the neosexual revolution deconstructs old forms of sexuality, models new 
relationship styles, and gender and sexuality preferences, yet is also steeped in egotism, an emphasis on difference, gratification, and the commercialization of sexuality.

The sexual revolution of the 1960s and 1970s, the changes made in the 1980s and 1990s, represent a divide between generations, between old and new ways of understanding sexuality (Martin, 1996). How then is the idea of sexual revolution different today, if we have seen changes in cultural and political norms around sex? What remains to resist or revolt against now? Given these histories, many of the claims for liberation made by sex-positive communities, are not original. It can be also argued that liberatory principles have always been coded for dominant, privileged voices. Also shedding light on the tensions inherent in seeking freedoms based on generalized ideals. Today, few scholars defend the work of Reich and Marcuse, critiquing elisions in theories of sexuality and sexual liberation that reify racialization and privilege. Yet, this demonstrates an absence of reconciling gendered and racialized theorizing in early work on sexual revolution. And demonstrates a valid reason to pay attention to these claims being made today.

The ideological reclaiming of sexual revolution, and the move to surpass theorizations of neosexual revolution, is where the subject of this study is situated. Sexual revolution or, sex culture revolution in contemporary terms, has been articulated as an opportunity to reclaim once perjorative labels like slut and pervert, for a new generation (Allyn, 2016:6). Affirming what Giddens argues, "What used to be called perversions are merely ways in which sexuality can legitimately be expressed and self-identity defined" (2013:179). Sex-positivity is claimed to have an important role to play in this process. Although the term was coined during the 1960s and 1970s sexual liberation movement, it 
has gained more mainstream attention recently. As I will show, the persistence of sexual shame is one way people may identify the need for sex-positivity with others, forming bonds over shared experiences. Public campaigns drawing attention to shame like SlutWalk, have tried to reclaim and normalize a woman's relationship to pleasure, as a recent example. The \#metoo movement has also introduced a public backlash against the shame of speaking up against sexual abuse. Both organized and proliferated through the Internet and social media.

To that point, it has been argued that, such questions can be explored in part due to Marcuse's theorizing that may still be relevant, "insofar as they direct our attention to the ways in which the relation of the Internet to society manifests competing potentials between, on the one hand, competition, exclusion, and exploitation, and on the other hand, cooperation, inclusion, and participation" (Garlick, 2011:223). In this way, Garlick (2011) argues that Marcuse's work is viewed as valuable for emphasis on the intersection of sex, technology, and capitalist economy, viewing his work as an opportunity to contribute to a critical theory of sexuality in the era of digital technology. Sexual revolution is thus being reformed in contemporary terms that acknowledge the importance of how people use the Internet and social media to connect. This is argued to in turn, influence the landscape of culture and society. As scholars have noted, "The Internet is not just a product or a presenter of sexual culture; it also shapes sexual culture" (Ross, 2005:349). This statement is reaffirmed in the data, which reveals that the necessary partnering with the Internet and social media technologies is crucial. Today, the influence of the Internet and social media enables an evolving understanding of the choices available to people sexually. This is key for the construction and proliferation of 
sex culture, especially across borders. Through these new media technologies, knowledge created and shared about sex-positivity and alternative sexual communities and practices has been said to assist in the evolution of sex culture. Thus, virtual spaces are fast becoming a place wherein bodies are articulated, managed, and surveilled, linking discourses of bodies and sexuality to "virtual and material spaces of everyday life" (Del Casino \& Brooks, 2014). This makes scholarly claims about sexuality and subjectivity more complex. In particular, social in/exclusion via technology and virtual mobility has also been demonstrated to promote more complexly power-laden ways to express sexuality and identity creation (Kenyon, Lyons \& Rafferty, 2002).

Not all freedom looks the same, however, and sex-positive worldviews can be interpreted as colorblind liberal reproductions steeped in privilege and white supremacy. Pointing to another problematic difference found in this case study around the subject's rebranding of revolution that prioritizes pleasure, bringing into focus the privileged limitations of such pleasure seeking. The assumption of being able to claim a sex-positive lifestyle remains a question of access. For whom is liberation and freedom afforded? Such elisions between theory (sexual liberation for all) and practice (actual liberation for all) identified in past movements, in many ways are reproduced and carried forward. Noting that "subcultures often inadvertently reinforce rather than subvert mainstream values, recasting dominant relationships in a subversive style" (Haenfler, 2004:407-8). This process tends to be reproduced within the racial politics of sex-positive communities, in which white liberal ethics grounded in privilege dominate the creation of affectual space. These days however, there is also a significant difference found in a "call out/in" culture. This strategy is idealized as using the surveillance represented by the 
Internet and social media to publicly or privately disrupt oppression and microaggressions that are glossed by colorblind racism and the reproduction of privilege.

With this project I respond to these gaps in the literature by investigating how racialized power and privilege are reproduced within liberatory sex-positive agendas. By critically interrogating the work that care does, I add to a discussion of its potentials and shortcomings: "care is equally the site of the reproduction of existing power relations and oppressions. Yet practices of care also generate new possibilities and communities, new (political) subjects and ways of relating to each other" (Tauqir, et al, 2011:18). This work proposes an alternative to theorizing of neosexual revolution, by examining how play and care contribute to neoliberal understandings of sexual freedom. To make this point, I refer to Giddens who states: "Sexual permissiveness is not at all the same as liberation" (Giddens, 2013:168). I go to explore how and why.

\section{Geographies of sexuality: Constructing sexual dissidents and cultural diasporas}

The geographies of sexuality scholarship that inform this project are concerned with the sexualization of culture and globalization of sex. I use these literatures to interrogate how power operates in spaces of play and leisure, underscoring a politics of safety, and the impact privilege has on sex-positive community. In this project, I am concerned with how power is articulated at a sex party and in sex-positive community, asking: who gets to play, who gets to care, and who gets to be transformed? Scholars assert that any potential for structural transformative power is diminished by a lack of "engagement with power" (Haritaworn, Lin \& Klesse, 2006:519). This is important to clarify the context because work on sexuality attentive to racial power is limited. With this case study, I contribute to this body of work by theorizing play as an important, yet undertheorized aspect of sexual 
intimacies; situating alternormative sexual lifestyles as dissidence; complicating the work sex-positivity and community-making does, particularly in response to how privilege and power operate.

Sexualization of culture, and the globalization of sex

Globalization of sexuality scholarship lays a foundation for the exploration of transnational disruptions within sex-positive community. Scholarly work to date regarding globalizing and transnational sexuality underscores the rifts in the KS "global" 12 community and events that contribute to discourses of transnational sexual politics. The term "transnational" here works broadly to "describe migration" and "transnational flows" that also "signal the demise or irrelevance of the nation-state" (Grewal \& Kaplan 2001:664). Suggesting also that a "borderless world" is one where culture is "more important or relevant than nations and that identities are linked to cultures" more so than to "institutions of the nation-state" (Grewal \& Kaplan, 2001:665). I understand such transnational flows of sexuality and sexual culture as being made possible by processes of globalization. ${ }^{13}$ Cultural diasporic identities are supported by the use of transnational here as well. More recently, scholarly investigations into the globalization of sexuality ${ }^{14}$ often take a queer postcolonial theoretical perspective. Drawing on the postcolonial, queer, anti-racist work of scholars such as Puar (2003, 2005), conceptualizations of globalizing sexuality are made more complex by efforts made to center race in queer, anti-racist, feminist politics across borders. ${ }^{15}$

As I have begun to articulate, current scholarship points to the Internet and new media technologies increasing the visibility of, and engagement with sex practices prompting anxious calls about the "sexualization" and "pornification of culture. ${ }^{16 "}$ To 
better understand the sexualization of culture, and the term sex culture I use throughout, I first take a look at how sex culture is theorized. Presently, sex culture is largely discussed in relation to youth cultures (Farrer, 2002; Nguyen, PA, 2007), hookup culture, sexpositive culture, and a NYU Press book series, Sexual Cultures, being the most prominent. This book series focuses sex culture on, to name a few topics, Queer Faith (Sanchez, 2019); Cruising Utopia (Muñoz, 2019); Queer Times, Black Futures (Keeling, 2019); and Sensational Flesh (Musser, 2014). As well, a sexual culture research group is housed at the Queen Mary University of London. ${ }^{17}$ Demonstrating that "sex culture," i.e. sexual cultures, are being theorized in differing terms. I read these engagements as moving toward an analysis of sex culture more broadly inclusive of black, brown, and queer folx voices. I go on to explore the representation of such voices in relation to the material, physical, and virtual spaces created by KS and its community, to uncover how and why spaces may take on the very 'qualities' that are given to such bodies" (Ahmed, 2007:156).

\section{Theorizing play}

To undertake such an exploration in the context of this case study that prioritizes play, I investigated early work on play by Huizinga (1949) and Norbeck (1974). These scholars did not consider adult play as useful in any sense, a mere indulgence. However, drawing on Woodyer (2012:316-22), I adopt the concept of play not as an event but rather a characteristic present in behavior. As such, more recently scholars have theorized that the ludic is bound up with "an embodied, expressive, and affective immersion in the moment" (Edensor \& Bowdler, 2015: 713). Creating temporary, fantastical spaces where one may be transported from the banal minutia of everyday life is an important aspect of 
what is said to drive transformation and strengthen community cohesion. There is an element of "flow" that Turner (1979) attributes to the liminal, which is also crucial to the production and outcome of KS events. Thus, applying understandings of communitas, another characteristic of liminality, adds depth to unpacking the affective investments made by and for KS participants. Communitas is about connection and I extend this to play; described as "the mutual confrontation of human beings stripped of status role characteristics - people 'just as they are,' getting through to each other" (Turner, 1979:471). And Woodyer (2012) insists further that, "the vitality or intensity experienced through playing adds strength and depth to our experience of the world. The affirmation and self-validation this engenders can prompt a generosity or openness towards the world that encourages us to be more responsive to others" (Woodyer, 2012:322). People claim to lose themselves in the moment and become immersed in their actions and environment (Turner, 1979:487; see also Edensor \& Bowdler, 2015). For Woodyer, play is relevant and enacted in the everyday, in politics, exceeding representation or rationality, and can engage critical reflection potentially prompting transformation. I argue that in these ways, play is one fundamental way in which care is enacted for this community.

\section{Sexual communities and non-monogamies}

The unpacking of what a cohesive community can be is equally instructive here. In order to achieve a reconceptualization/theorizing of sexuality and play, I am inspired by Jeffrey Weeks (1996). Theorized as a "sexual community," the KS community is organized firstly along common interests of individuals that ascribe to similar social values, particularly regarding sexuality. The community is valued as a place to be heard or be held, often prompting praise for being "there." Such mainstreaming of what was 
once singularly at the margins, begs for an investigation into changing perceptions and stigmatization (Hutzler, Giuliano, Herselman \& Johnson, 2015). Therefore, sexual community defined as having: "community as a focus of identity; community as ethos or repository of values; community as social capital; and community as politics" (Weeks, 1996:73), is an instructive way I understand what community means in this case. This is relevant as I explore the care work undertaken by community to remediate stigma and shame, via playful connection.

Through mainstream media sex culture, polyamory and consensual nonmonogamies (CNMs) are gaining attention. Accordingly, this study is informed by theorizations of polyamory and CNMs, kink and BDSM, as new types of sexual orientations, or what Robinson (2013) calls a "strategic identity." Scholars have also taken up the project of deconstructing sex-negative language to understand what sexpositivity is and why it is important (Glickman, 2000). Discourses on sex-positivity have touched upon the legal, where scholars expose and challenge the law's unspoken assumption that sexual pleasure has negligible or negative value, and examine how rejecting this assumption requires a reconceptualization of several areas of law (Kaplan, 2014). Many of the respondents in this case, and others I spoke to invested in sex-positive community generally, claim that strategic identities help them form insular bonds that mediate social and cultural pressures to conform. These new orientations regarding nonnormative sex propose the need for greater legal protections, for instance, to help reconcile employment discrimination (Tweedy, 2011). Such a move would render “polyamory intelligible within dominant political and legal frameworks of sexual diversity" (Klesse, 2014:81). 


\section{Sexual dissidence and citizenship}

In response to the changing ways sexual diversity is conceptualized, this case then also offers a fresh perspective on sexual dissidence. Sexual dissidence is a concept that has largely been used to refer to lesbian and gay identities, also referred to as "dissident sexualities" (Bell, 1995; Binnie \& Valentine, 1999; Wilkinson, 2011). Scholars refer to lesbian, gay, and "other" sexual dissidents but very few are specific about what or who these "others" are (Binnie, 1997). ${ }^{18}$ This scholarly interest both reflects and prompts a wider popular interest in radical sexual practice. For the purpose of this dissertation, such work has both detailed and informed the subsequent analysis with regard to the social life of these sexual practices, and their work to disrupt, transform, or calcify, wider cultural attitudes about sex and sexuality. From BDSM and kink to orgies, polyamory, consensual non-monogamy, and public or communal sex, sex-positive community members view these activities as a means to develop and maintain meaningful relationships. What I will show is that by bringing together this scholarship, through this case we can get at the consequences sexual dissidents face on a daily basis. I am contributing to the scholarship that is beginning to investigate non-normative relationships from an anti-racist, postcolonial lens that complicates understandings of intersectionality when applied to such alternative lifestyles (Haritaworn, Lin \& Klesse, 2006). Particularly interesting for the focus of this study is the argument Sheff \& Hammers (2011) make regarding people involved in kinky, perverted, or alternative sexual practices and lifestyles. They explore the consequences of such behavior and lifestyle choices, documenting affiliation between polyamory and BDSM, and identifying a reinforcement of homogeneity among class and 
race. So to, for the community I studied, polyamory, BDSM, kink, and fetish are dominant modes of relating intimacies.

As such, sexual dissidents are the focus of much of the scholarship on sexual citizenship, particularly focused on LGBTQ politics and mobilities. Citizenship in this sense, is understood as bounded by a "moral majority" that circumscribes what is available and to whom, where punitive measures are taken to the extent of exclusion or worse. Key tools for acquiring citizenship vis a vis political representation are to agitate for it and enforce visibility using "strategies of acting up," especially when operating contrary to dominant hegemony (Bell, 1995:143). “The 'good citizen' is heavily responsibilized, meaning that only certain formations of sexual culture can by parlayed into citizenry" (Bell \& Binnie, 2006:870). This is problematic in this case where citizenship is also conceptualized within chosen families and non-normative relationships. Therefore, citizenship contextualized through care is at odds with what it means to be a good citizen (Hubbard, 2001) outside the safety of sexual community. This project makes further interventions to demonstrate how class and race are articulated when these practices are bound up in sex-positive community. Sara Ahmed (2007) considers "whiteness as a category of experience that disappears as a category through experience, and how this disappearance makes whiteness 'worldly"" and to "repose the question of whiteness as a phenomenological issue, as a question of how whiteness is lived as a background experience" (Ahmed, 2007:150). This is how I perceive the claims of sexual liberation as potentially tone deaf regarding what liberation and freedom may mean for non-white bodies. Yet, scholars also caution that whiteness studies and the marking of the unmarked norm poses a danger "in identifying features of 
a dominant white epistemology, whiteness is endowed again with the transhistorical, essential, asocial and universal character of unmarked whiteness" (Nash, 2003:640). Privilege is revealed to be increasingly harmful when it comes to sex, especially if colorblind affirmations deny that race matters (Doane \& Bonilla-Silva, 2003). And the ability to succeed in life with a bootstrap mentality that is implicated in liberation ideals displaces and obscures white advantage through "attribution error" (Doane \& BonillaSilva, 2003:190). Furthermore, visceral geographies (Hayes-Conroy \& Hayes-Conroy, 2010a) explored further below, suggest that such background experience for some, particularly with regard to pleasure is internalized as dangerous and inaccessible. Gaps observed in the literatures

These forerunners inspire a new look at questions of sexuality and citizenship, urging deeper investigations of transnational studies of sexuality. How has the conceptualization of sexual citizenship changed? What has instituted such changes? And how do we theorize sexual citizenship now? Inspired by Richardson (2018) I question whether the constitution of sexual citizenship is also the constitution of normative sexuality? Must one be evaluated as having normative sexuality to obtain citizenship rights today? Grewal and Kaplan continue, arguing that "A more interdisciplinary and transnational approach that addresses inequalities as well as new formations can begin more adequately to explore the nature of sexual identities in the current phase of globalization” (Grewal \& Kaplan, 2001:664). Especially relevant, are claims that progressive identity politics serve to connect sexuality and social movements, across space (Grewal \& Kaplan, 2001). In fact, Grewal and Kaplan argue for the need to "begin to understand how the study of sexuality remains bound by disciplinary constraints" 
urging for a "more interdisciplinary and transnational approach that addresses inequalities" in how "sexual identities" may be constructed globally (Grewal \& Kaplan, 2001:664). Few scholars have investigated sexuality and social movements at national and transnational scales (Grewal \& Kaplan, 2001; Povinelli \& Chauncey, 1999), suggesting an important theoretical intervention for my work.

\section{Interventions and contributions}

It has been argued more broadly that "sexual citizenship is most commonly associated with non-normative sexual practices and identities" (Bell \& Binnie, 2006:870). Such previous scholarly efforts have extended the work on sexuality beyond the Global North. This work is also situated in the examination of alternative sexual practices in the Global North, which lends itself to expanding discourses of sexualization of culture and globalization of sex. As I have shown however, none of the previously mentioned literatures have been much concerned with sex parties as worthy of sustained attention. This project extends the work being done in geographies of sexualities ${ }^{19}$ by demonstrating how sex play is intertwined with care in service of an activist-oriented agenda aimed at changing sex culture. The sex-positive community studied reproduces itself in formulaic, yet uneven ways across the spaces it occupies. Interrogating this case from a globalization perspective provides a more robust understanding of transnational sex culture. Here I go further and argue that sexual citizenship exists in opposition to rights, privacy and bodily autonomy, to be fought for, and is "about enfranchisement, about inclusion, about belonging" (Weeks, 1998:39,48). The ideas of belonging, desire, and the erasure of racialized privileges influence the creation of sexual community transnationally. With this case, I thus answer the call to investigate sexuality 
transnationally via sex culture and play parties, ${ }^{20}$ attempting to center race as "a necessary optic when considering kink" (Cruz, 2016:4), and more deeply interrogate the impact privilege has on liberatory or kinky scenes. This study offers a fresh perspective on how sexual communities are formed and sustained, and explores the reproduction of structural inequalities that have historically excluded certain populations from enjoying a kinky or perverted lifestyle.

\section{Playing with a politics of care: Toward an affective, sex culture activism praxis}

\section{Affect, emotion, and intimacy}

The feminist geography scholarship that is central to this work has to do with theorizing care, pleasure, the body, and queer space. I am intrigued with how identity and community are constituted by and through sexual politics across space to contribute to discussions of affectual and queer sex. A politics of emotion (Ahmed, 2014; Wilkinson, 2009) is therefore inscribed in this project where intimacy is fundamental to understanding the importance of communal sexuality to the community studied. Therefore, this project is informed by feminist, queer work on affect, emotion, and intimacy. I put these theoretical strands into conversation to strengthen the contribution I make to exploring how sexual play and care operate upon community-making and proliferating sex-positive cultural agendas. Scholars have investigated emotions, affect, intimacy, and touch within geography to address their internal, often intangible geographies. ${ }^{21}$ Where the theorizing of affect and emotion has been conceptualized as distinct categories (Thien, 2005), or has been critiqued as being inseparable and intertwined (Pile, 2010). Responding to this, I assert that affect, emotion, and intimacy 
are concepts that suggest how we respond to encounters creates an impression that is left and enacted through feelings (emotions), which align us (or not) with others.

These theoretical foundations are important for this project, and make possible an intervention that answers the call for such reconceptualizations. I go further to claim that these qualities also inform one another on a visceral level (Hayes-Conroy \& HayesConroy, 2010a). J. Hayes-Conroy \& A. Hayes-Conroy's (2010a) “visceral geographies" provide added scaffolding to this project by extending and relating affect, emotion, and intimacy to social politics and change in three ways: "first, visceral geographies advance understandings of the agency of physical matter, both within and between bodies. Second, visceral geographies move beyond static notions of the individual body and toward more contextualized and interactive versions of the self and other. And third, visceral geographies encourage a skepticism of boundaries by insisting on the imagining and practicing of our (political) lives in, through, and beyond dualistic tensions" (2010a:1273). Taken together, this means that affect being an impression made upon a body, between bodies, and emotion being the reactive manner in which individuals and collectives respond to such impressions. In this case, such reactions and responses are often mediated through care practices.

\section{Theorizing care}

The ways in which I apply care are influenced by scholars theorizing geographies of care as: social justice, welfare, and human rights; questions of social exclusion and health care; and gender/ethnic divisions of care work and labor. ${ }^{22}$ But I contend that this is nothing new and not where the uniqueness of this study is found. The fight to make care work visible as sexuality activism was brought to national attention during the AIDS 
crisis. ${ }^{23}$ The care practices developed at this time helped to agitate for desperately needed access to medical and social services for people dying of AIDS (Evans, Gosse \& Moser, 2003:209). HIV prevention campaigns initiated by members of gay communities geared toward "safer sex," condom use, and the creation of safe spaces, are evidence of care practices in the service of such sexuality activism (Boyce, et al., 2007:2; Escoffier, 1998, 2008). Furthermore, AIDS buddy volunteers were a type of support system that provided care work theorized as developing new terrains for citizenship (Brown, 1997:129). "Combined with the ongoing restructurings of the family, AIDS caregiving opens up the possibility that the citizen can be found in the home and family" (Brown, 1997:26). The development of chosen family and alternate kin structures is an important feature for sexual dissidents. With the case study of KS, respondents talk about the feeling of "home" and "chosen family" that is made possible by hard won agencies for sexual rights and cultural change fought for in previous movements. Adding that, LGBTQ relationships "should be viewed as an alternate form of kinship in contemporary life" (Brown, 1997:121). Alternate forms of kinship can therefore be established as a type of care work, relied upon for support, particularly for sexual communities.

\section{Sexuality activism}

Consequently, such past and current sexuality activisms have concentrated on AIDS/HIV awareness and health care, the push for normalization/decriminalization of sex work and sex workers rights, sexual health rights, gender politics, and understanding violence against gendered bodies. ${ }^{24}$ Scholars have investigated how such movements and communities have acted to change policy, normalize gender fluidity, sexual preference, and fight for autonomy over how to express sexuality and with whom. ${ }^{25}$ More recently, 
activists push for the construction of "polyamory as a sexual orientation, arguing that some people are immutably predisposed toward forming multiple relationships" (Robinson, 2013:21). Even more, queer theorists such as Wilkinson (2010) urge the placing of non-monogamies into a broader queer political agenda, aiming to see rejection of monogamy as a political act. In doing so, the potential impacts of consensual nonmonogamies become a critique of broader social structures. These literatures come together to help me make sense of and establish the context for the contested spaces upon which KS participants navigate their sexual lives. Yet, questions remain about what theorizing care can do for embodied sexual intimacies.

\section{Failures of care}

The literatures that frame past movements also help me contextualize in/exclusion of sexual dissidents in terms of care and failures of care. The ways care has been previously engaged with calls into question the radical potential of what sex-positive communities suggest they have the power to do. In this case, intimacy and care also serve to help individuals process grief and shame, remembrance, inclusion, and memory that is integral to the experience of transformation that many of the respondents speak about. At the same time, this work has led me to questions around transformation: who gets to experience transcendence, and how. Shame and care are thus linked through affective registers of intimacy and connection. More importantly though, "shame makes privilege visible to us and function as reminders to researchers of sex and sexuality why, just as sexual shame is a persistent phenomenon" (Mercer, 2018:1306). In response to this, I contend that this case helps to demonstrate the importance of reevaluating transformation 
claims in terms of care, also asking about the privilege of who gets to seek out and experience the transcendence of shame.

Care can also be constructed as a "term through which intimacy and labour are configured" (Cooper, 2007:243). Therefore, I also reflect on what Lawson says are "the changing realities of who has access to care and who does care work" pointing "to ways in which care work is privatized and devalued" (Lawson, 2007:2). Which also helps to shed light on how shame and stigma are managed as the focus of some care work for sexpositive community members. But, care ethics are said to question "neoliberal principles of individualism," (Lawson, 2007:3) emphasizing a move to communal responsibility, integrity, and unifying care rather than privatizing it. This is an important point about care that suggests higher ideals like integrity and communalism are part of the utopic, liberatory vision of some sex-positive community members. Therefore, care can also be more affective in register as sensitivity, trust, generosity, empathy, and commitment, being action-oriented, featuring responsibility, responsiveness and attentiveness (Cooper, 2007). This is how I will demonstrate in the chapters to follow that connection, education, and information sharing are conceived as care work for this sexual community.

\section{Gaps observed in the literatures}

Sex culture activism and ideals of revolution may not be new, and may be perpetually hindered by the reproduction of power imbalances in the same or emerging ways as past sexual revolution iterations. However, I argue that such activism works to continue putting pressure on changing the discourse around sexual freedoms and expression. This case then demonstrates that sexual autonomy and bodily agency remain 
contentious, political sites. However, to date, feminist geographies scholars have yet to undertake a serious analysis of the globalization of sex culture happening via play parties or sexual communities. This is an important intervention to bring our attention to the impact sex culture has on individuals' lives and choices. Sexual oppression, racialization, and marginalization are layered and intersectional, complicated more by "perverse" or freaky sexual behaviors and preferences. Therefore, an important way that the case of KS differs from the examination of past movements is by digging into the emotional component of sexuality and community.

\section{Interventions and contributions}

I investigate sex culture more deeply to reveal how the excavation of such emotional terrain is often fraught with trauma regarding sex, as my respondents will speak to. Even when sex is presented as positive and filled with personal responsibility and agency, people feel shamed for having multiple partners, or making other nonnormative lifestyle choices. They claim to feel oppressed by the moralistic judgments underlying such choices sociohistorically (Barker, Gill \& Harvey, 2018). This case will show that such risk affects family and relationships to the state and institutions, job loss and economic risk, and potential for social exclusion or marginalization. This study contributes to the literature on sexual politics to demonstrate that the seeking of pleasure or the right to pleasure may also reproduce an uncritical view of what pleasure is. I investigate the privileges associated with pleasure that echoes a bootstrap ethic I discussed previously, foreclosing the realities of what pleasure is and whom it is good for. Finding that the visceral, embodied experience of intimacy and sex play, helps build the affective bonds of community, and at the same time, demonstrating that bonds of 
community are not evenly felt or accessible to all. In response to this, I provide a discussion about how I am also changing the terms of how care is conceptualized via my case study. Because, I hope to show that "care is not simply a form of doing; it also has an emotion dimension, captured in the idea of mattering" (Cooper, 2007:255). For this case study, bringing together emotion and care through playful intimacies demonstrates further how and why things matter.

An investigation of the spaces KS constructs can help further understandings of sexual subjectivity, citizenship, and how race, class and sexuality intersect. This work is in service to challenging the uneven construction of sexual liberation ideals and realizations; and works to reimagine what subjectivity and sociocultural change could be. Therefore, I present a case that helps us conceive of how dissident sexualities, feminist care ethics, play, and Internet technologies, inform, hinder or potentially create new avenues for connection and advocacy to unfold.

\section{Research Objectives}

In order to answer these fundamental questions, I interrogate the sexual politics of sexpositive community across space. I investigate Kinky Salon's brand of care ethics that are informed by embodiment, affect, intimacy, or what is considered "heart-centered" relating established by communication and consent, resulting from the creation of safe space and striving for inclusivity. A study of this subject investigates how play is used to destigmatize sex and instill an ethics of care. Care is recast as prioritizing play, leisure, and community, through sex, education and information sharing, which shows up as community-making practices. Participants at the heart of this study ranged in age from early 20 s to 60 s, many identifying as white, with about twenty-five percent of 
respondents identifying as BIPOC. Many respondents claim to be invested in alternative kinship and family structures built upon sex-positive community and consensual nonmonogamies, tending toward queer and hetero/bisexuality among the LGBTQ spectrum. Furthermore, often respondents are situated as part of what has been termed the "creative class" (Pratt, 2008), which is enmeshed within education, professional, activist, artist, and self-employed roles. This class type is instructive to the way KS and sex-positive community in general is interacted with, as these types of sexual communities pay capital to adapting creative new ways to be in the world. For these participants, such practices are idealized as transformative, and I want to know how and why. What does such transformation look like and is it happening at the scale of the individual, community, or farther afield, as this community tries to establish a transnational identity? The following research questions guide this project:

Ch1: Theoretical question: What is so political about sex play, or playful sex? Empirical question: What are the challenges inherent in striving for inclusivity in sexpositive community? Does Kinky Salon's vision of playful sexual revolution create change? For whom and how?

Ch2: Theoretical question: What are the implications for care practices to create sociocultural change around sexuality today? Empirical question: How is an ethics of care enacted at a play party? Within sexual community? How do care practices contribute to sex culture activism for community members?

Ch3: Theoretical question: How does sex-positive community operate transnationally? 
Empirical question: How does the case study of Kinky Salon disrupt or inform discourses of transnational sexuality and sexual citizenship?

\section{What is Kinky Salon and how does it work to influence sex culture?}

"Kinky Salon is a global sex culture community trailblazing the path for the Sex Culture Revolution. We promote sexual liberation by hosting community gatherings where sex is integrated into the social fabric of the events. Kinky Salons are [...] really fun parties with costumes, art, dancing, and performance, as well as areas where people can be playfully sexual. Creativity is the focus of the events, and sex is just one way to express yourself at a Kinky Salon" (KS Companion Manual).

But is any of this new? Sexual revolution is not a new concept. The claims of sexpositive communities, and in this case, are focused on fighting for many of the same grievances expressed during the sexual revolution of the 1960s, 1970s, and 1980s during the AIDS crisis; in fewer or similar ways, and perhaps to less effect, consequence, and gains. So with the case of KS, I investigate why people in different locations attest to the power of sexual play, particularly in a sex-positive community, and why this is seen as revolutionary now.

Originally developed in San Francisco in 2003, Kinky Salon is an event that emphasizes liberatory sex culture, fostering community-making rooted in participatory, DIY ${ }^{26}$ culture, and based on the founder's experience in the fetish, sex-positive, kink, and Burning Man ${ }^{27}$ worlds. Kinky Salon has been in operation for over fifteen years, expanding from its birthplace in San Francisco to include locations in sixteen cities in the Global North including: Austin, Barcelona, Berlin, Copenhagen, Denver, Las Vegas, 
Lisbon, London, Los Angeles, New Orleans, New York, Paris, Portland, San Francisco, Seattle, and Toronto. All these events are sanctioned by the founder and subscribe to the organization's seven core values: playful, safe(r), inclusive, creative, community spirited, socially conscious, and sexually progressive. ${ }^{28}$ The Kinky Salon event is claimed to not be an orgy, but billed as a fun party to explore one's sexuality creatively and safely. ${ }^{29}$ Kinky Salon events weave the reality of the lived experience of sexuality together with an uninhibited use of the absurd:

Guests danced and played into the early hours of the morning with increasingly less pieces of their character in place. As I moved through the rooms dancing and taking in the scene, I could view the play happening all around, not just sex, but naked people laughing, joking, trying new things, engaging with other people, even while engaging in sex. Conversation and playful behavior permeated the low changing light and cheeky music of the playspace. One guest remembers seeing a pair of giant monster feet hoisted into the air, seeming to wave with rhythmic movement to anyone watching.

Oftentimes, those new to the scene have an expectation or curiosity about what takes place at a sex party. But most soon find out that the emphasis is not centrally focused on sex. According to the founder of KS: "it's not like sex clubs that are all about power and power play and everyone playing a role [...] that's a playground for sure, but it's quite a limited one $[\ldots]$ and that's available at Kinky Salon but it's not the focus of it" (Interview, February 2014). Kinky Salon events are structured to produce momentary spaces that encourage consensual play and humor, which also support sexual openness and exploration. At these events, fabric, lighting, comfortable play spaces, and an 
abundance of safer sex supplies, are an important feature of creating the temporal space and ambiance for the night. Participants enjoy the silly, playful themes and costuming the party is known for, performing characters that projects one out of their normal reality. Others find that the KS community allows for experimentation with unconventional relationship structures such as polyamory and consensual non-monogamy. In the early/formative years of KS, the founder used to joke that the intention was to spur the next sexual revolution and sex-positive world domination. These days, Kinky Salon as an organization positions itself as a vanguard of these ideals within an increasingly popular consciousness - with the ambitious aim of heralding a new age of sexual revolution. According to the founder, "Kinky Salon is all about hope, love, the future and creativity and community and collaboration... it has a massive amount of hope" (Interview, February 2014). To achieve this sense of hope, Kinky Salon's organizational structure has evolved from the house fetish party it was when it began more than fifteen years ago.

Despite what many participants claim are the cultural norms constraining bodies and sexuality, KS community members place a great deal of importance upon having a space to experiment with different ways of relating to others. Humor is thought to defuse the tensions of engaging in sex. Being an "agent of chaos" is articulated by the founder as the philosophy guiding the community and work that it does in the world. The political nature of creating of new ways of being in the world for organizers, uses sex, humor, play, and ridiculousness coupled with intimacy and care to deconstruct old ideals and spur growth. Many informal conversations with participants also point to this being a time in which cultural discourse around sexual ethics, consent, and alternative lifestyles have dovetailed with popular events such as Burning Man (BM) ${ }^{30}$ which emphasizes 
mutuality, cooperation, and inclusivity. These qualities set the tone for achieving the goals set toward conscious social engineering, for many, but certainly not all, who participate. Nevertheless, principles such as these are important to many participants who often expressed their involvement in politics, organizing, information sharing, and education within the realm of, or adjacent to sexuality rights. There are some notable actors involved in the KS community, such as sex worker activists, trans and genderqueer activists, and kink/BDSM, relationship anarchy, polyamory and consensual nonmonogamy practitioners and advocates. These community members are situated as grassroots advocates for sex worker rights, speak out about consent culture, educate on gender nonconforming identities, and body positivity. In each of these participants' own worlds, they are doing the work of initiating discourse around matters of sex/uality. Such involvement in sexual politics helps make involvement with KS and their "sex culture revolution" mission an entangled aspect of one's personal political sphere.

The KS organization is able to capitalize on this timing by integrating sex-positive philosophy with an amalgamation of affect-inflected strategies that increase capital and ideological reach among sexual subcultures. Primarily as a volunteer run organization that eschews commodification, KS was conceived early on as an outgrowth of the same underground art scene in San Francisco that birthed the Burning Man Festival. ${ }^{31}$ The founder and many of the early community members and volunteers were also active members of the Burning Man community in the early 2000s. Early Burning Man and its core principles have influenced the essential character of KS's sexual ethics and community politics, progressive or problematic as they may be. Much of the attraction has to do with the rules for behavior. Over time, many of the BM ideologies that were in 
alignment with KS's development became integrated into the KS ethos; also prompting the founder to "give Kinky Salon away" in the spirit of open source sharing. Noting that this type of tech-oriented language permeates cultural, artistic and social movements and collectives that originate in the San Francisco Bay Area. As I will show, new technologies are integral to the spread and sustainability of sex-positive culture, also further cementing a bond between sex culture and the "creative class." Furthermore, because I noted overlap, or spillover, between the BM scene, Kinky Salon, and sexpositive communities in general, it is important to also note the potential challenges inherited from the adoption or adaptation of co-opted ideologies.

Because of this overlap, many of the sociocultural critiques of BM may also apply by extension to KS due to their points of ideological imbrication. For instance, one respondent regards the people at Burning Man as: "People who think like me, dress like me and act like me and I like it. I love my tribe" (Interview, 2014). This underscores a sense of how "tribe" is associated with the "home" like qualities of alternative communities, with a return to communalism (St John, 2018). Another notable similarity is what privilege looks like for sex-positive, kinky communities. Being black at Burning Man parallels what POC respondents have stated is common to experience from sexpositive events. As an example, in the film, In Pursuit of Happiness: Black at Burning Man (Levy, 2018), ${ }^{32}$ interviewees state that Burning Man has been considered a "white people thing." And although it is a common trope that BM is a place people go to be free, this idealized freedom is problematic for many people of color. People claim that coming from the "default" world, histories of violence and oppression continue to inform cultural, structural and institutionalized racist practices that may be reproduced at BM. 
The cognitive dissonance in being welcomed "home" to a place that might feel unsafe is unappealing to many POC. With this in mind, posing an increased risk for the participation of people already marginalized by drug stereotypes, is the widespread use psychedelics. The supposed freedom achieved through the expansion of the mind with psychedelics is considered rather from a perspective of danger that is more deeply ingrained into the everyday psychology reported by people of color. A history of developing respectability politics to compensate for varieties of racism is then at odds with the radical self-expression ideals of the BM ethos, including the fraught terrain of black sexual politics. The silliness, playfulness and levity of radical self-reliance and creative expression is complicated by histories of survival that equate to such radical selfexpression being dangerous. However, participants claim that undoing racism is also about taking up space for self-determination and the exploration of the boundaries of selfhood, including spaces of pleasure. Yet, being greeted with a "one of us" type of tribalism (St. John, 2018) (i.e. we are all Burners here) is entrenched in a liberalized colorblind acceptance of being a "burner." In the film it is claimed that racial microaggressions are diminished or not as apparent in the BM world, however. Pleasure then lends to a quality of life that enables one to "experience more possibility," and "dream bigger."

While this potential is certainly available, the comments by people of color in response to this film largely revolve around the cost being too high at $\$ 400$; tickets incredibly difficult to get even if one has the money; a lack of interest in going to the desert to be uncomfortable for a week in harsh conditions surrounded by mostly white people; and art produced by (and for) white consumption. Such liberatory ideals are a 
target for the reproduction of inequalities and privilege, which will be demonstrated as problematic for the KS community as well. When it comes to pleasure and sex, privilege is marked as an "embodied racial power" because "all actors socially regarded as 'white' ... receive systemic privileges just by virtue of wearing the white outfit whereas those regarded as nonwhite are denied those privileges" (Bonilla-Silva, 2017:193). This is reflected in the complex intersections of danger, desire, pleasure, and self-determination referenced in the film above. This also reflects some of the same criticisms relayed to me about KS and sex-positive community by people of color.

However, for those compelled to sexual adventuring, sex-positive communities are increasingly accessible. Over fifteen years, the Kinky Salon community has evolved its event into a formulaic structure that can be recreated in approved locations, given a creative commons license (open source, or information sharing technologies). ${ }^{33}$ In other words, those who want to have an event in their town may adopt the KS formula. A creative commons license granted by the organization enables others to produce the event given certain conditions: crediting Kinky Salon in all virtual and print material; adhering to the use of the Charter and the PAL system; using the volunteer system; drawing up an agreement to uphold safety standards for safer sex practices and the general well-being of the community attending the event; and agreeing to the general oversight of the Kinky Salon "Mothership." ${ }^{34}$ Kinky Salon San Francisco (KSSF) is known as the "Mothership," the originator, and the seed from which all other local chapters were born and modeled after.

The first step to starting up a Kinky Salon in your town is to fill out the online application which can be found on the website. For those who have not attended, they are 
urged to do so in order to have first-hand experience about the event and what goes into creating the KS "vibe." In order to have an application approved, all KS chapters agree to model their events on the Kinky Salon experience, meaning how things are structured and operate should not deviate from the formula set forth in the manual in order to be sanctioned as an official Kinky Salon. Local chapter agreements are expected to be understood and adopted. When a new location is approved, whether it is in rural Kansas or a major city, ${ }^{35}$ one of the new core team members will sign a contract agreeing to the KS core values, and as a signifier of becoming part of the KS Global Community (KSGC). ${ }^{36}$ As a member of the KSGC, each team has access to the organizers and volunteers from other chapters to share information and advice; has a listing on the main KS website; has permission to represent the event as an official KS; and has access to the founder and advisors for consultations via email or video conferencing. For those wishing to open their own local chapter, they are urged to understand: "Opening a Kinky Salon means that you're part of something bigger than just your parties. Acknowledging the origin of Kinky Salon will give your event credibility, and help increase awareness of the Sex Culture Revolution of which you're a part" (KS Companion manual). The "Companion," or production manual, is made available to all new chapters and details how to run a local chapter including information and advice on pre-event, event production, and post-event tasks that are supposed to make a KS successful and fun. To help organizers and volunteers do this, it covers everything from: the intangible "setting intentions" for the space and the night; to the more concrete aspects of running the show; how to select performers and run a cabaret; what needs to be done during the evening by all the volunteers; how to budget and break down the space; online and offline 
communications; to team building and internal/external conflict resolution practices. In general, new producers are urged to understand:

"We live in a complicated world when it comes to sex culture, rife with paradoxes, fears, heartache and trauma. Kinky Salons encourage exploration and experimentation in a consensual, inclusive environment, run entirely by teams of volunteers. Kinky Salon is a social experiment embedded with tools to help people explore their identity, learn to communicate their boundaries, and surf their comfort zones” (KS Companion manual).

This is important to understand because it is the space and vibe that is intentionally created for each KS event that spatializes aspects of care, play, and intimacy said to be transformative for participants. Building upon what works and changing what does not is said to be a labor of love for organizers and participants. The manual then offers tips and advice for producers and volunteers, for community building, as well as some absolutes regarding the event formula. It also reassures new teams that the KS headquarters, or "Mothership," and Kinky Salon Global Community are always available for help and guidance.

Community-making practices are thus a focal point for the care work being done by this community. The manual is explicit about the use of community volunteers. It suggests that producing a KS with volunteers is not about saving money, although for many chapters that is a concern; it is about building community. For the founder and most of the core volunteers, this is not a money-making venture, and it is only in the last several years that some producers are taking an income from Kinky Salon. Every team structures their finances differently however. Some teams take no pay and circulate any 
profit into producing the next events, storage, décor, etc. Other teams pay their core team members a portion of the profit, circulating the rest back into the production of the next event and maintenance.

Despite the need to reproduce formulaic events, management of teams is constructed as decentralized for the organization. The organization's "Mothership" takes a hands-off approach with teams, putting an emphasis on autonomy by maintaining vestiges of BM ideals like self-reliance, civic responsibility, and participatory communal effort. Unless they ask for help or seem to be getting derailed in some fundamental way, the core teams are organized as self-managing teams, meaning that there are no hierarchies or direct management. It is thought that this enables everyone to have a say in the conversation and decision-making processes. This structure is supposed to hold the onus of responsibility on each member to be committed to a collaborative process. In doing so, team members are supposed to understand and encourage non-violent communication strategies be utilized. This structure also states that each location's team will be autonomous in managing their budgets, creative process, and timelines without interference. Although, determining who needs help and how, has proven to be highly subjective, and teams are relied upon to speak up to voice their concerns.

As the cohesiveness of the community has been solidifying over the years, the organization's "Mothership" has started to establish new ways to support the global teams. Beforehand, teams could go on for a while without reaching out for help if they were experiencing challenges of any sort. ${ }^{37}$ However, with more events coming online in recent years, the "Mothership" has begun to architect new structures for supporting 
teams. Training materials and communication strategies are continually revised with participation and input from all the global KS chapters.

\section{Chapter summaries}

Following this first chapter, in the second chapter, METHODOLOGY \& METHODS: The embodied researcher in a sexualized field, I first provide a narrative of how I arrived at this dissertation topic, leading me to the research questions guiding this work. Then I discuss the feminist theory in methods that have provided the framework for data collection and analysis. I show that working through the lenses of affect, emotion, intimacy, and care were fundamental to how I engaged with the data and my presence in the field. Then, I provide a methodological discussion using authoethnographic excerpts to situate myself as a feminist researcher in a persistently sexualized field. I reflexively examine how my presence impacts informants and research outcomes, including how I cope on a daily basis with my positionality and the effects that this work has caused. I discuss the care work needed to conduct sensitive sexuality research, allowing an autoethnographic vulnerability to demonstrate these tensions. Following this, I lay out the methods used to collect data over a two-year period.

In chapter three, THE POLITICS OF PLAY: Negotiating power, connection, and transformation in a sexual community, I focus on exploring a sexual community (Weeks, 1996), unpacking the politics of sex-positive adult play. I show that sexual politics can be used to center play as a vehicle to destigmatize sex. I situate this case among sex-positive culture and discuss what that means, and how this community envisions the work of pushing boundaries. Specifically, I demonstrate why play is important to the success of this sex-positive community, and reveal how the politics of play operate (Woodyer, 
2012). I show how sex-positivity and cultural change agendas drive the community but are not without their troubles, which I highlight. This revised revolutionary agenda challenges interpretations of the neosexual revolution by prioritizing heart-centered care ethics, pushing forward a new way of envisioning sex culture activism. In doing so, I show how such ideals versus practice are bound up in identity and class-based politics that complicate the "liberatory for all" objective.

In chapter four, CONSTRUCTING CARE IN SEX CULTURE: Reimagining revolutionary change one educated orgasm at a time?, I demonstrate that feminist care ethics are engaged and mobilized as activism through community-making, education, and information sharing. From pure enthusiasm, to working through sexual trauma, the power of coming together to be sexually expressive in socially non-conforming ways is a strong feature reported by many participants. I show that to do this, sex-positive education, workshops and socials, focuses community building as part of the mission to create sociocultural change. Thus, affect and intimacy figure into the care-based work to achieve community cohesion. These liberatory care ethics can be problematic however, as the reproduction of privilege and bias is not resolved through them necessarily. Moreover, privilege is demonstrated as a matter of choice among those who can practice such ethics.

Chapter five, TRANSNATIONAL INTERROGATIONS OF SEX-POSITIVE CULTURE: Globalizing a privilege of perversities, interrogates the spread of sex culture transnationally. I return to a discussion of the transformative nature of play and show that the transnational reach of community helps facilitate change in how sexual subjects and sexual citizens are constructed. However, doing so demonstrates where inclusivity ethics 
are complicated by what happens when play is not play for all. I discuss how tensions and challenges are mediated, highlighting the consequences of a sometimes clumsy or imperfect, yet reflexive, reconciliation of racialized and class-based politics. Finally, I discuss some of the policy changes being made by this organization as a way care ethics are recast to articulate anti-racist policies, behaviors, and language in sex-positive community.

In the final chapter, CONCLUSION: Learning from failures: Deconstructing privilege to create a more caring agent of chaos, I review the data, which demonstrates that sex-positive community benefits from cohesion and support provided by togetherness and education, centered around play and sex. The motivation to develop and revise how community is reproduced stands in contrast to legacies of sexual revolution of the past, and how they are being differently carried forward. This work underscores the productive and problematic ways transformation is conceived of in connection with others, to sociocultural politics and activism, and sexuality. I show why it matters that scholars engage with how sexual dissidents construct their subjectivity around alternormative sexuality, often dictating how one engages with the world. I show how the spatial negotiations fraught by the constructing and remaking of identity are influenced by globalizing sexuality proliferated through the Internet and social media. I have found that community members are bound together through affective investments and emotional labor to insure care for each other. And, found complications arise around questions of racial exclusion, marginalization, and aggression, which are further complicated by egalitarian idealism. However, efforts to reconcile care blind spots have 
recentered marginalized folx who are motivated to dismantle and recreate sex-positive community structures that prioritize their needs.

${ }^{1}$ Such radical, public, and kinky sex is the focus for what I consider alternative sexual practices, or non-normative sexuality (For non-normative sex practices, see: Berlant \& Warner, 1998; Califia, 2000; Herman, 2017; Sheff \& Hammers, 2011).

${ }^{2}$ Sex-positivity has been used as a framework for understanding adolescent sexuality (Harden, 2014); power, politics, and performativity (Gedalof, 2000); how it has contributed to a politics of transgression investigated by feminist and queer theorists to refocus attitudes toward pro-sexuality (Glick, 2000; Queen \& Comella, 2008); and calls for revising what it means to be sex-positive and the type of freedom that relates to (Fahs, 2014).

${ }^{3}$ For a discussion of changes in attitudes around what was once considered taboo sexual practices: Comella \& Sender, 2013: 2563; Garlick, 2011:222; Howe \& Rigi, 2009:298; Megatron, 2015; Steele, 2015.

${ }^{4}$ Shortbus (2006) is an independent film featuring LGBTQ characters. The plot of this film engages with complex themes - centering sex as a pleasurable thing that should be embraced, talked about, made humorous, and seen as a creative and productive force, which the movie suggests many are out of touch with today.

${ }^{5}$ For public sex, radical sex, sex publics, see: Albert, 2011; Berlant \& Warner, 1998; Califia, 2000; Castiglia, 2000; Dangerous Bedfellows, ed., 1996; McGlotten, 2013.

${ }^{6}$ Adopted from the language of BDSM fetish events, the term "play party" is more commonly used to gesture to an event where kinky and sexual activities occur and are not discouraged. The term "play" in these settings almost singularly refers to engaging in (consensual) sexual activity with others.

${ }^{7}$ Alternormative sexuality (Cooper, 2009) includes a growing interest in practices including, but not limited to: polyamory (consensual non-monogamy), ${ }^{7}$ swinging $^{7}$ (or partner swapping), kink, and Bondage and Discipline, Dominance and Submission, Sadism and Masochism (aka BDSM) (Barker, 2013; Weiss, 2011). In recent years, this is an area of study that is beginning to gain momentum as an important aspect of sexuality studies.

8 "Sex culture revolution" and the agenda of bringing "sex out of the shadows" are concepts that have been used by the KS organization to articulate their mission. References to these ideas have been documented in numerous interviews and informal conversations, on the KS website, and other media (www.kinkysalon.com). 
${ }^{9}$ Grove, Parsons \& Bimbi, 2007; Halkitis, Parsons, and Wilton, 2003; Hurley, 2009; Mimiaga, Reisner, Bland, et al., 2009, 2010, 2011; Solomon, Halkitis, Moeller, et al., 2011.

${ }^{10}$ For discussions of affect, emotion, and intimacy, see: Ahmed, 2004, 2014; Bondi, 2003; Davidson, Smith \& Bondi, 2012; Dixon \& Straughan, 2010; Pile, 2010; Povinelli, 2002; Thien, 2005; Valentine, 2008. For care ethics, see: Atkinson, Lawson \& Wiles, 2011; Bondi, 2003; Green \& Lawson, 2011; Lawson, 2007, 2009; Massey, 2004.

${ }^{11}$ Sexual revolution: Allyn, 2016; Bailey, 2002; Escoffier, 2003, 2008; Evans, Goss \& Moser, 2003; Giddens, 2013; Haenfler, 2004; Martin, 1996; McKay, 2005; Wouters, 1998.

12 I put "global" in quotes because Kinky Salon is not currently everywhere around the globe (i.e. the Global South). However, I include the use of global to acknowledge that Kinky Salon conceives of itself as a global community, i.e. the Kinky Salon Global Community. The KSGC is a network of all the sanctioned KS events, so far, located only in the Global North. This serves to also signal the globalization of sexuality processes that I outline in the literature review. The KSGC is also inclusive of the transnational KS community. Community members are often encouraged to seek out local KS chapters and events when traveling to those cities KS's are located.

${ }^{13}$ For globalization of sexuality, see also: Altman, 2002; Binnie, 2004; Gorman-Murray, 2006; Puar, Rushbrook \& Schein, 2003.

${ }^{14}$ Altman, 2002; Binnie, 2004; Gorman-Murray, 2006; Puar, Rushbrook \& Schein, 2003.

${ }^{15}$ Petzen, 2012; Puar, Rushbrook, Schein, 2003; Puar, 2005.

${ }^{16}$ For sexualization and pornification of culture, see: Attwood, 2006, 2014; Comella, 2014; Gill, 2012; Paasonen, S., Nikunen, K., \& Saarenmaa, L., 2007; Smith, 2010.

${ }^{17}$ https://sexualcultures.wordpress.com/

${ }^{18}$ Scholars have made reference briefly to sexual dissidents in discussing BDSM practitioners (Barker, 2013; Bauer, 2010; Herman, 2007; Lenius, 2011; Weiss, 2006, 2011; Wilkinson, 2011).

${ }^{19}$ For reviews of geographies of sexualities, see: Binnie \& Valentine, 1999; Browne, Lim \& Brown, 2007; Curran, 2005; Houlbrook, 2001; Johnston \& Longhurst, 2009.

${ }^{20}$ For transnational analysis and sexual politics, see: Briggs, McCormick \& Way, 2008; Grewal \& Kaplan, 2001; Povinelli \& Chauncey, 1999; Manalansan, 1995. 
${ }^{21}$ For affect, emotion, and intimacy, see: Ahmed, 2004, 2014; Bondi, 2003; Davidson, Smith \& Bondi, 2012; Dixon \& Straughan, 2010; Pile, 2010; Povinelli, 2002; Thien, 2005; Valentine, 2008.

${ }^{22}$ For geographies of care, see: Atkinkson, Lawson \& Wiles, 2011; Beasley \& Bacchi, 2007; Clement, 2018; Cooper, 2007, 2009; Datta \& Lund, 2018; Fisher \& Tronto, 1990; Massey, 2004.

${ }^{23}$ Boyce, et al., 2007; Brown, 1997; Escoffier, 1998; Parker \& Aggleton, 2003.

${ }^{24}$ See: Blasius, 2001; Brown, M.P., 1997; Johnston, 2016; McCracken, 2013; Nash, C.J., 2010 .

${ }^{25}$ See: Davis, 2009; Fox \& Ore, 2010; Price, K., 2010; Seidman, 1995; Warner, 2000.

26 "Do it yourself" - a creative work ethic that is proudly embodied by many in the KS and Burning Man communities, among others.

${ }^{27}$ Burning Man is an annual art and music festival that takes place in a remote Nevada desert. This event is community focused and strongly emphasizes radical states of being such as decommodification, gifting, and self-reliance, to name a few core principles. Many of the early KS adopters were active in the BM community.

${ }^{28}$ http://www.kinkysalon.com/companion/2015/06/19/the-7-core-values-of-kinky-salon/

${ }^{29}$ www.kinkysalon.com

${ }^{30}$ https://burningman.org/culture/philosophical-center/10-principles/

${ }^{31}$ Burning Man core principles are: radical inclusion, radical self-reliance, radical selfexpression, decommodification, gifting economy, communal effort and civic responsibility (www.burningman.org).

${ }^{32}$ https://www.youtube.com/watch?time continue=2\&v=IpBzqH8RowU

33 "You are free to: Share - copy and redistribute the material in any medium or format Adapt - remix, transform, and build upon the material for any purpose, even commercially. This license is acceptable for Free Cultural Works. The licensor cannot revoke these freedoms as long as you follow the license terms. Under the following terms: Attribution - You must give appropriate credit, provide a link to the license, and indicate if changes were made. You may do so in any reasonable manner, but not in any way that suggests the licensor endorses you or your use. ShareAlike - If you remix, transform, or build upon the material, you must distribute your contributions under the 
same license as the original. No additional restrictions - You may not apply legal terms or technological measures that legally restrict others from doing anything the license permits" (https://creativecommons.org/licenses/by-sa/4.0/).

${ }^{34}$ A term used by the founder to refer back to the original system began by the founding team in San Francisco. Also referred to on the website as the "Kinky Salon Mothership," the San Francisco location is where KS started, and what all the other global events look to as their point of reference. All the KS organization's policies and communications come from the founder, or Mothership, which is also default for KSSF.

${ }^{35}$ There is no limitation for where a KS might start up. For instance, the Austin event used to be smaller such that one of the members' two-story Victorian home served as the venue. And the NorCal event is held in a rural mountain town known for its hot springs and hippie vibes.

${ }^{36}$ The Kinky Salon Global Community is the name given to the network of all sanctioned Kinky Salon local chapters that are spreading "globally".

${ }^{37}$ Some of the big challenges experienced by teams are unavailability of venues, falling through or being too costly, inability to produce any income and therefore self-funding the event (which can become expensive and stressful), difficulty staffing the core team or finding volunteers to help on the day of the event, internal communication difficulties and personality clashes. 


\section{METHODOLOGY \& METHODS: The embodied researcher in a sexualized field}

The playspace was very big, with 20 full-sized, raised beds (the team makes sure to have raised beds in order to facilitate accessibility for differently-abled guests). And at the height of the playspace's capacity there were as many as 100 people, give or take, all playing at the same time. There was no extra space to sit or lay down, and getting in and out of the space was challenging. Pretty intimidating, all those people having fun. Just a peek, but, wow.

"Knowing that with so much time having passed, and diverting attention to the care work needed to sustain, I maybe could have done more. Is it laziness? Is it healing and self care? Is it all rooted in fear of the unknown future? Probably a bit of all these things" (Mountz, et al., 2015).

\section{Introduction: A project in the making, journeying to the topic}

In this chapter I begin with a background of how I arrived at my subject, which led me to the research questions that developed this project. I then discuss the methodological theories underpinning this work. I reflect upon how affect, emotion, and intimacy shape knowledge production, and the feminist care ethics that have shaped this project. Finally, I move on to show what was done, with whom, how and why.

This project has gone through several evolutions to become what it is today.

Originally I was interested in digging into the politics of interracial relationships during the Harlem Renaissance period in New York. During early literature reviews, I became aware of buffet flats, which were underground spaces and secret places assumed to be safe for people to be transgressively sexual and creative. ${ }^{1}$ However, the rent parties and buffet flats of the Harlem Renaissance have received much deserved scholarly attention. Although, what I found also compelling about these spaces was how art and creativity were called upon to liberate sexuality through freedom of expression. In these spaces where racial, sexual, and gender politics were entangled with and enacted partly through 
creative expression - people could flagrantly embody taboo. I became very curious and wondered "Where are these kinds of spaces now?" I decided to investigate contemporary instances of places people create to relate to each other, through common threads of sexuality, art, and transgressive liberation ideals. I became aware of sex-positive sex parties and communities doing the work of irreverent sex culture creation. I narrowed my focus to Kinky Salon (KS) as a contemporary event and community that seemed to exemplify these ideals.

\section{Feminist methods in geography: Coming to terms with intangible geographies}

The methodological ethos central to this project is informed by feminist theory and praxis, with a particular emphasis on geographical work on affect, emotion, and intimacy. ${ }^{2}$ In fact, feminist and queer geographers' innovations in these methods have guided this work. Methodological interventions distinguishing reflexivity and positionality, ${ }^{3}$ autoethnography, ${ }^{4}$ intimate insider/outsider relationships in the field, ${ }^{5}$ the embodied researcher, ${ }^{6}$ sexuality in research, ${ }^{7}$ and care ethics, ${ }^{8}$ were crucial to understanding my presence in the field.

First, I understand that "sexuality and space are mutually constituted...where sexuality is not fixed or static, but shifts and changes depending upon politics, place, and space" (Johnston, 2015). As a researcher this was a very challenging aspect of this project. I did not anticipate how I would also be constructed as a subject in these spaces and influenced by the fluctuating terrains between sexuality and space. Returning to the internal, reflexive space to process encounters and emotions, transformed the researcher into sexual subject, also informing this project. The relationships I developed were forged and strengthened through frequent contact and an unexpected need that I become 
vulnerable and share some of my story. Though never pressured for sex or treated disrespectfully, I was continually reminded of my positionality as a researcher, and also as someone feeling overwhelmed by the continual focus on sex. What this instigated for me personally were feelings I had never known to question before about my sexuality and how I relate to others, in itself daunting. In doing so, sometimes out of sheer exhaustion or pushing my comfort zone talking, thinking, and doing sex research, circumstances forced me to stop resisting care on the basis of fear of not doing enough. Highlighting also the ambivalence around feelings expressed in the quote at the beginning of this chapter. I was made to embrace the real-world implications of care: from others and to the self, which shaped how I perceived myself, and this project.

Another way this project is methodologically grounded is through the use of the Internet. The Internet and social media are elements that are woven throughout the narrative of this study. Through the technologies available in an increasingly networked world, the Internet makes possible the exchange and proliferation of sexual culture. This kind of accessibility emphasizes the growth of globalized sexuality. ${ }^{9}$ It has also been used as a tool for the excavation of data, and it is a tool that is used foremost by the Kinky Salon organization and community to carry out an agenda promoting sex, fun, and connection. Therefore, with this research I demonstrate that the Internet as a research method is particularly useful to sexuality research today given the abundant sex resources online from pornography to online communities like FetLife, ${ }^{10}$ a social network for "the BDSM, fetish and kinky community." With this project I will show that the Internet is necessary for such communities to thrive. Therefore, this project and Kinky Salon's work could not be done without it. Ross affirms this, arguing: "The medium creates new 
situations that require description, such as the question of what is actually done, the language of its doing, the mode of action, and the contribution of the Internet to the psychic states and meanings associated with cybersex and sexual shopping” (2005:351). I argue this also holds true for KS in building and maintaining their sexual community. Ross goes on to state that the Internet enables us to "appreciate the way our 'gaze' at sexuality occurs through the electronic filter of the Internet - and how it may also enable us to see things at wavelengths not previously visible" (2005:351). Accordingly, in this study - by my use of the Internet - I show that KS organizers make use of the Internet as a facilitator providing access to community resources and information. Thus, the Internet is of primary importance to this project as it is a fundamental mode not only of conducting research but also of how the subject proliferates the work it is said to be doing. ${ }^{11}$

The above methodological, conceptual frames ultimately ground this project's empirical findings and the ways in which the research was conceived, carried out, and finally analyzed. In fact, scholars argue that through the lens of affect we can obtain new perspectives on "ethics, the complexity of social life, the place and enactment of power, the circulation and articulation of difference, and the politics of grounded material relations" (Woodward \& Lea, 2010:155). This played out for me directly when I was cared for by my friend "Enormvs," a contact made through my research with Kinky Salon. Over time, I was observing, participating, interviewing and producing events with him. Which called into practice all the things I hoped I had learned about maintaining professional ethics and an objective distance in the field. As the quote above notes, the complexity of life and various ways power is enacted through the creation of affective 
bonds, shifted my relationship with a subject. When I had three surgeries including a bilateral mastectomy and reconstruction at the end of my field research, "Enormvs" was there to keep me company, help me cook, clean and even pack up my apartment. I paid him for his help, but he accepted whatever I could offer. This demonstrates how these visceral, embodied, and affectual geographies are where play and care become integrated into intimacy and sexuality. Care, intimacy, connection, and affective relation are what I contend make this methodological process interesting, with a fresh perspective to contribute to the field. These elements are central to this intellectual narrative.

Furthermore, I employ such feminist methodologies to identify these types of emotional entanglements in fieldwork, such that I may reckon with and constantly reevaluate my positionality and power relations implicit in fieldwork.

\section{Sexuality research and self-care: Understanding fieldwork as a site of deep reflexivity}

Often feeling laden with heavy pressures, I have been conscious of maintaining privacy of informants, staying the course with the work, and responding to the many shifts this project has taken through data collection and what seems like endless writing. In doing so, I am further impressed by the need to reveal how affect, emotion, and intimacy shape knowledge production through such feminist care ethics that have shaped this project. As I have foreshadowed above, and Longhurst et al. (2008) suggests, there is an emotional toll and process of doing and being researchers that has been undertheorized. The importance of centering affectual registers with regard to how I engaged with the field and interlocutors was ever present. Because these qualities are prioritized within this community's organizing practices and participant involvement, they became fundamental lenses through which I operated in the field. In particular I focus on three connected 
themes concerning self-care as it relates to the research process: reflexivity, boundaries, and managing stigma. Therefore, I explore the personal nature of fieldwork and argue for the necessity of deep reflexivity (Ellingson, 2006). I suggest that creating boundaries in fieldwork helps mediate the stresses involved. With a specific focus on how my health shaped this project, I underscore the difficulties in navigating intimacy and emotional entanglements in the field, to highlight how researchers can manage the stigma or shame that can be attached to doing sensitive sexuality research.

To explore these themes I have had to reflect on how I approached the field and how I conducted the research. I used autoethnographic writing to reflexively situate myself in a complexly sexualized research process, both in and out of the field. Paying attention to feelings and emotions that arise during knowledge production cannot be divorced from the process of doing and being in the field and beyond. In order to help ease these feelings, daily journaling as part of the writing process, helped offset the fear of misrepresenting my field experience. This practice became an integral part of my personal and academic journey. Additionally, feminist scholars Kaspar \& Landolt (2014) argue that only a few scholars ${ }^{12}$ have engaged with sexuality in written reflections on fieldwork (2014:108). Furthermore, Ellingson (2006:299) advocates for deep reflexivity during methods analysis and write up, suggesting that "the erasure of researchers" bodies from conventional accounts of research obscures the complexities of knowledge production and yields a deceptively tidy account of research."

Keeping a journal was helpful for my mental and professional health. The use of autobiographical writing helped me to expose the anxieties around concerns of concealment and revelation. But, in doing so I also felt the discomfort of becoming 
vulnerable, submitting myself to the scrutiny embedded in the embodied and intimate experiences I have had as a scholar. Even so, it has been an extremely valuable tool helping me work through tough situations in the field, and even tougher emotional and sometimes physical entanglements with self and interlocutors. Moreover, Cupples argues, "if sexuality both produces space and permeates social life, then the fieldwork experience is no different" (2002:382). This suggests that it is valuable to include autoethnographic narratives drawing on all senses and drawing connections to research processes, which I do periodically throughout this text.

Therefore, I have had to explore what feminist care ethics mean for the researcher as well as the researched. But, I have often questioned the validity of the insertion of the personal within the institutional space proscribed by the research and writing process. Through reflections on these processes of bodily enactment, the researcher may scrutinize the body, bodily movements and the actions (Mol, 2003) that follow up these corporeal and intellectual internal battlegrounds.

I guess it's just a product of what I am going through on a personal level. It is so heavily bound up in the day to day of what I'm working on that it is hard to even see one without the other. I believe I'm getting better, I see changes. This is a good thing for now. However, I am quite a bit more tired these days.

Including autobiographical writing like the above, within this process focuses on this enacting body and how it has responded to the pressures of doing sensitive academic work. Consequently, autoethnography and the accompanying vulnerability it produces are enacted upon my fatigued body (Moss \& Dyck, 1999), and yet provide some sort of comfort in complete transparency. 


\section{Creating boundaries: Negotiating intimacy and emotional entanglements in the field}

The stress of fieldwork can be something that I found best dealt with by attempting to create healthy boundaries for myself the researcher, and the subject. According to Cuomo and Massaro (2014), "boundary-making serves an important role in creating emotional and physical distance between our participants, our field sites, and ourselves that can prove beneficial even in contexts when the researcher might not consider themselves an 'intimate insider"' (2014:103). Similarly, I felt a need to "create physical and emotional boundaries to construct" myself as a researcher "in the eyes of... participants" (Cuomo and Massaro, 2014:95). My particular boundary making processes were necessitated by complicated breast and thyroid cancer diagnoses I received as I prepared to conduct fieldwork. Because of a variety of overlapping pressures, I chose a radical path to healing opting for alternative healthcare methods. By rejecting standards of care, I was told I was crazy for listening to my body, and became angry with what I viewed as corrupt, limited, and quality-of-life diminishing options for healthcare - determined to find alternatives and do it my way. Learning about and implementing these methods was a full time job, as it turned out. I became hyper-conscious of all things that went into and surrounded my body, which amplified my need for physical boundaries, affecting my work. During this process, my positionality became even more complex and lonely. At the time I did not have a partner, so I believed it was necessary for me to remain celibate in order to maintain vigilance over my immune system. I became opposed to sexual contact or expression, and many forms of intimacy.

I have no plans of getting it on with anyone. I don't even have any plans of kissing or making out with anyone. Actually, the idea of someone touching me, or worse 
yet, tonguing me down with their nasty tongue, does nothing for me. I have it set in my mind that people in general don't take very good care of themselves. Or rather, because of the discipline that I implement in my life around food, exercise, and spirit energies, it is challenging to find someone that is in alignment with those values. In that case, I would feel much less reluctant to make out with a vegan, or raw vegan that exercises regularly, doesn't eat processed foods, or put otherwise toxic chemicals into their body on a regular basis.

A sex researcher disgusted by bodies and sex, how ironic. In retrospect I understand that I was not judging bodies, but more accurately, very angry about my personal experience and perceived limitations.

Scholars urge researchers to rethink the boundaries of the field as related to the researcher who co-creates boundaries particularly when navigating social relations (Smith, 2014). With regard to something more innocuous (in my circumstances) such as flirting in the field, these researchers found that "positionalities shifted in the course of interactions" (Kaspar and Landolt, 2014:108). According to Kaspar and Landolt (2014) it is important to include various shades of sexual performances, such as apparently harmless flirtation, into our reflections on data collection. But my entire field site was always inflected by sex and sexuality. As a result, I often spent quite a bit of time concerned with how I would engage with others in the space at the events, before each event, often producing various levels of anxiety. I found my positionality shifting due to the turns my healthcare took throughout the data collection process. My presence in the field began very much as a detached participant observer, looking for volunteer tasks to take up time and mark my presence in the space as useful, necessary, and legitimate 
(Kobayashi, 1994). I really could not tolerate even being touched by someone who could potentially introduce illness to my already compromised immune system. But I used my assumed role as a researcher and an event producer to insulate me somewhat, rather than deal with the possibility of sex. I always had a job to do, and could be found attending to the needs of the people, or the space, to create a boundary that protected me from delving too deeply into the intimate. Newmahr (2008:619) illustrates "the value of incorporating subjectivity into traditional ethnographic analysis" demonstrating that insider/outsider status is often influenced by the changing positionality of the researcher to the researched. Yet, I had not allowed that process to unfold more organically for me due to the limitations I had placed on myself under the guise of healthcare.

The academic at this point is personal so it makes it even harder to know where to begin... the boob... I must admit that I am still interested in the presence or company of another. But all the shit that comes with it! My gosh. Dark night of the soul - yes, again, this boob. What a crazy lesson you have been.

The decision to remain celibate while doing immersive work with sex and sexuality both enabled and inhibited my fieldwork. I remained serious, professional, detached. Flirting felt foreign and disingenuous. I was able to keep myself at what I considered a legitimately ethical safe distance from engaging in sex relying on the excuse of my condition. But what I understand now is that celibacy became a coping strategy for maintaining a distance that was both professional but also personal: first due to the uncertainty of how engaging in sexual activity might affect my health; and also my emotional and physical safety. At the same time, I surrendered any option to more 
directly interrogate how those ethics operate for a researcher in a sexualized field, or even make those choices at all.

It's funny that every time I feel lonely or want company, I get the opportunity to test that desire out, without the commitment (in my role as event producer).

Certain actual physical limitations narrowed my research focus even more as I had planned on doing a comparative analysis between $\mathrm{KS}$ and other communities that produce creative, themed, sex-positive play parties. Ultimately, I did not have the stamina and energy for this kind of data collection, concentrating instead on an in-depth ethnography of the KS event and community. ${ }^{13}$ Overall, I spent two years in the field, longer than I had wanted, more or less due to my inability to move at a more rapid pace, balancing my healthcare needs and physical limitations with the work I was doing. It's days like this I just have to take it super slow... infusion day. Be kind to myself; don't get caught up in what other people are doing and what I'm not doing.

As the above demonstrates, during this process, I have often fretted over the dense personal and political engagements with doing such sensitive work. I have been specifically challenged with the anxieties of how to let relationships organically unfold, versus when to say good-bye to those who would become friends and momentary colleagues in a co-creative process. And scholars are beginning to pay attention to these types of dense emotional entanglements in fieldwork. Particularly for feminist researchers, these must be identified and reckoned with in order to be constantly reflexive, reevaluating one's power relations implicit in fieldwork (Laliberté, N., \& Schurr, C., 2015). 
To demonstrate this point, I reflect on the personal and professional rapport that I developed with the KS founder. Our relationship became defined by reciprocation, creativity, good communication, and friendship. Early on I discussed with her my intention to see as many Kinky Salons as possible. I then mentioned that I wanted to see what they looked like in Europe as well. This conversation sparked an idea for an interactive European KS tour. We decided to go together on what was dubbed the "Euro Kinky" tour in March and April, 2015. Apart from the first KS that I attended as a mere participant-guest, I had spent most of my time in the field shielded from certain interactions through the role of volunteer and event producer. However, once again I became a participant-guest on this KS Tour. On the European leg of the tour, I played the role of road manager, with the founder and I taking meetings and making notes of people wanting to be part of the global vision. All told, we were on the road, sleeping in shared beds, and basically doing everything together, for a month. As a result, I felt somewhat insulated and protected against wandering aimlessly and knowing no one at these events. But by the end I had developed friendly relations with many US and European KS producers through the KS Global Community (KSGC) ${ }^{14}$ These relationships helped relieve some social anxiety. Although, the anxiety and stress that preceded these relationships was at times paralyzing.

Feeling pangs of missing the interesting people I became momentarily bonded to on our journeys. I feel a hollowness where I was filled with loving relation to positive energy. And even in my romanticized remembrances I stay aware that it is so hard and at the same time so easy, to connect. 
Still, there is a struggle to find a place for emotions in research that is not relegated to the research diary, but reflects the need to understand "embodied fieldwork." Laliberté \& Schurr (2015) argue that "To attend to emotions within research is to attend to the ever shifting social landscapes in which we and the knowledge we produce is embedded" (2015:74). Of course, I reflected on the connections I made, realizing that even though I am a researcher, I am also human and prone to attachments.

Our other friend has been diagnosed with colon cancer. She called me to see if I could talk to her, offer her some support or any information I have on healing practices, diets. We all decided to take a girls trip to the hot springs to decompress and give each other naked hugs.

Coinciding with the European tour I visited as many Kinky Salons as I could also in the United States to interview producers and participants (visiting eight of the twelve local chapters established at that time). I was approaching better health and was perhaps open for different encounters. Up to this point in my research I felt that something was missing. Rose (1997:316) argues that the researcher is, in a sense, completed, "not by what she knows, but what she uncertainly performs" (Diprose et al. 2013). I wanted to experience the playspace, fully immersed in the event, and how one might otherwise engage with people at these parties. I felt that understanding was missing from the work I had been doing. Yet, I still shielded myself from the physical and emotional vulnerability of engaging in sex, or intimacy beyond flirting at these events.

And what part to play does this breast dis-ease have? What power does it really have over me? Of course I want to minimize it as if it's no big deal, and then again, maybe that sends the wrong message. In any case, I do feel blessed to be 
on this journey, having met some truly incredible and lovely people, people whom I'd actually like to keep in touch with over time.

\section{Managing the stigma and potential for shame in doing sexuality research}

As my journal entries indicate, working to balance self-care while undertaking sexuality research has been challenging. Aside from health challenges, I also faced a number of moral/ethical challenges due to my investigation into taboo sexual practices that sometimes had negative impacts on close personal relationships. According to Israel (2002), motivations for undertaking research in sex are always scrutinized, judged, and sometimes ridiculed (2002:257). In fact, scholars explore “the 'field-generated stress' of

managing an identity as a researcher in sites of stigmatized, sometimes sexist, underground or illegal activity" (Comella \& Sender, 2013:2566). It is true, the impacts of such work can be personally devastating. I have had a partnership and a number of friendships end based on a misperception of what my work is and why I am doing it. I have had people make assumptions about my sexuality and make claims about me being deviant and by extension a bad person. These types of reactions felt like deeply personal attacks. Therefore, managing stigma (Israel, 2002:258) becomes an important coping mechanism for both the researched and the researcher.

\section{Feminist methods in action}

As I mentioned in the Introduction, the film Shortbus instigated many questions and curiosity to arise about alternative, kinky, sexual lifestyles. Among the film's apparently layered objectives, it explores the complex interrelationships between individuals engaged in sex-positive community. It centers the connections between art, creativity, and sexuality as co-creative processes. Intrigued by some of the themes in the film, I then 
wanted to know more about whether these types of utopian-esque sexual communities actually existed. With a focus on art, sexual politics and liberation, what are the possible achievements or limitations of such collectives? Therefore, the questions guiding this research, as recalled from the Introduction are:

Ch1: Theoretical question: What is so political about sex play, or playful sex?

Empirical question: What are the challenges inherent in striving for inclusivity in sexpositive community? Does Kinky Salon's vision of playful sexual revolution create change? For whom and how?

Ch2: Theoretical question: What are the implications for care practices to create sociocultural change around sexuality today?

Empirical question: How is an ethics of care enacted at a play party? Within sexual community? How do care practices contribute to sex culture activism for community members?

Ch3: Theoretical question: How does sex-positive community operate transnationally? Empirical question: How does the case study of Kinky Salon disrupt or inform discourses of globalizing sexuality and sexual citizenship?

To fully explore the these questions, this project evolved very clearly into a multisited ethnography of one group that throws "arty, sexy parties" where sex also happens. As the research unfolded I was inspired to investigate the productive potential of sex play and claims of creating sociocultural change. This interest was deepened by the opportunity to bring an intellectual focus to sexual community that is oriented as transnational (Falzon, 2016). I began in New York as the starting point. I explored sexpositive communities and play parties that had adopted what seemed like similar ethics 
for safety and fun, only to hone in on Kinky Salon as distinct. I then volunteered as a Kinky Salon New York event producer and stepped into the role of what it is like to participate in creating sex culture. As I mentioned earlier, having done early research on other groups and events that were also claiming to be sex-positive play parties and communities, became important to then situating $\mathrm{KS}$ as among a phenomenon taking place in sex-positive culture more broadly. From there I decided it would be important to experience as many KS events as possible, that were established at the time of data collection. Qualitative methods were used, specifically: participant observation, semistructured and unstructured interviews, autoethnographic reflections, and content and critical discourse analysis for visual, printed and online materials, including photo and video.

My fieldwork and data collection was broken up into three phases (which I did not originally plan). First, I began to understand the field and build networks, which took some time as I was living in a new place with no previous contacts. During this first phase I drastically changed the direction the research was headed, shifting my focus from a comparative analysis of sex parties in New York, to a multi-sited in-depth ethnography of Kinky Salon. At this time I also began recruiting interviewees. Second, I engaged with participant observation by volunteering as an event producer for a KS in New York City. This position was vital to understanding the inner workings of the organization and its politics. I also continued to interview participants and organizers at this time. And finally, I went on a tour to visit eight of the twelve events established in the US and Europe. This tour is what served to connect all the pieces of data together, creating a more robust analysis of a transnational sexual community. 


\section{Trust building}

As I was seeking interviewees, it became quickly apparent that it would be a challenging task due to the sensitive nature of discussing sexuality. To be able to understand how identity politics operate within sex-positive community, it was my objective to speak to people identifying as among a broad range of identity categories. This objective, however, was not so neatly realized. Many people I contacted were suspicious, cautious, or somewhat hesitant to participate until we discussed consent, insuring privacy would be maintained indefinitely. I then became keenly aware of the necessity of taking a step back to firmly establish my networks to help the interview selection process. I developed relationships with the founder, organizers, and volunteers, by email and in person, prior to and at events. This helped facilitate opportunities to interview participants, volunteers, and producers from each local chapter that I visited. In fact, trust building was a crucial element for the beginning phase of research that extended throughout the process.

Without trust I could not have proceeded with this research, especially given the ethical considerations involved in doing sensitive work with people who might feel too vulnerable speaking about their sexuality. Similarly, Breitbart (2010) points out that by "behaving ethically, we maintain public trust. From that position of trust we may be able to continue research and to do so without causing suspicion or fear" (2010:37). This is where sharing part of my story became useful. In fact, reciprocation is an affectual register that came up often during my research, proving my willingness to be interested in the subject through an offering of my own. This was reflected by participants that commented how it makes them feel to know I care (things said to me about caring, during 
interviews: about them, about sex culture, putting this work out there in the world, making a difference for people who live this way). Furthermore, building a solid foundation of trust enables more successful adjustment to unexpected events or turns the research may take (Breitbart, 2010:153). Another key way I built trust with informants was by demonstrating my involvement in the community by volunteering as a KSNYC team member. This enlightened, yet complicated my positionality even more. At times this work felt more like a twist on participatory action research (Cahill, 2007), in that I was working within a collective that was producing events but with a broader message of social change. Thus, what started as a more distanced, conventional approach to participant observation evolved into a more hands-on observant participation (Brown, 2007) by going to events, volunteering as an event producer, producing photos, videos and graphic materials promoting events, and subsequent travel to see other local chapters and meet volunteers and community members. This positionality proved crucial to developing a multi-faceted understanding of this organization and sex-positive culture in general. By becoming more integrated into the community I was legitimized as invested. I established trust with the founder who was supportive of this work. I then made it well known to organizers and community members that I was an academic researcher investigating what a sex-positive play party is and why it is important to a global community of people. As a result, I was vouched for by fellow volunteers, who then referred additional interviewees. Overall, the time and effort it took to gain trust and nurture relationships allowed me to gain access to participants and data, and therefore present rich, thick ethnographic descriptions (Crank and Cook, 2007). 


\section{Interviews}

In order to answer my research questions interviewees were asked to share often deeply personal and intimate topics. During this time, I conducted fifty-three semi- and unstructured interviews, with many additional hours of informal conversations. Overall, interviewees were selected by purposeful, convenience, and snowball sampling predicated on me getting to know community members. Thus, one limitation of this research is in the sampling, as I was limited to speaking with people who I came into contact through the sex-positive, play party, and Kinky Salon communities. Through word of mouth I was able to recruit interviewees from within the organization including event participants, producers, and volunteers. I also placed advertisements on my personal social media pages to recruit people interested in talking about participation with sex-positive communities and Kinky Salon. Our discussions ranged from personal philosophies on sex, sex-positivity and polyamory, to participation in public sex, Kinky Salon and other sex parties, and critiques of sex-positive community.

The interviews conducted were helpful in fleshing out nuances for each of my research questions. I wanted to hear from those individuals who had experience with sex culture, sex parties, and/or alternative lifestyles and practices. This engages with the first and second theoretical questions I pose: What is so political about sex play? And, what can we understand about social change through sex play? Furthermore, I wanted to understand people's views of Kinky Salon, sex-positivity, polyamory and nonmonogamies, sexual identity, care and play. I wanted to understand how KS may or may not be a catalyst for experiences that shape community members' opinions and understandings of sexual politics, for personal and cultural change, and why this is 
important. Change for community members is evidenced through growth opportunities afforded by having access to education, information, and community. These concepts are all identified as aspects of the "sex culture revolution" that KS builds its image upon, and further addresses the second theoretical question: What are the implications for care practices to create sociocultural change around sexuality today? Additionally, I wanted to investigate the impact of the KS ethos in action, by focusing on the impact of the Internet and media technologies on sex culture. Answers to these questions were also particularly insightful for understanding the third theoretical question: How does sexual community operate transnationally? And, how does sex culture activism, which is influenced by care ethics, operate across space? Ultimately, I investigate the claims that taken altogether, support the outcomes of the KS brand of playful sexuality for participants as a new wave of sex culture revolution.

The majority of the respondents were in their 30s, some in their 40s, with a few in their 20s, 50s and 60s. Many identified as low-middle class, being in the arts, education, or self-employed; and those that identified as middle-upper class, being employed in corporate or other industry-related fields. Several respondents identified as students, professors and professionals. There were almost twice as many female identified respondents as male identified respondents, one trans-person, and one two-spirit identified individual. There were about as many heterosexual as bisexual self-identified respondents, and several queer and gay identified folx. Most of the respondents identified as white or Caucasian, with about a quarter of the individuals identified as "people of color," "black," or "mixed." These self-reported identity categories make up the interviewee tags, which are taken from the respondents' own words, rather than 
coalescing the diverse responses into less descriptive categories of my choosing. I have chosen to include their own identity markers to remain consistent with how those in the community view themselves, which is in alignment with the agency and autonomy striven for within the KS ethos, thus, what the community is used to and feels comfortable with. ${ }^{15}$ These descriptors help to become familiar with the ways these community members regard sex culture and their position within various institutional and sociocultural structures. This also provides more depth as to the intersectional nature of identity constructs in relation to the world.

\section{Content and data analysis}

The ethnographic data collected while in the field details how the events are structured and what happens at the events; investigates the lives of people that are involved in sex-positive community and what that means; and probes how the community restructures understandings of non-normative sexuality and how that contributes to sex culture. Transcribing and coding approximately fifty-three interviews was an overwhelming task. In order to help me with the initial processing of the recorded interviews, a transcription service was used. This service was selected based on its promise of upholding privacy standards and the signing of a non-disclosure agreement. I decided to use this service because at that time I was undergoing multiple surgeries and was fearful of not being able to continue on with my work for an undetermined amount of time. As I discussed previously, the effects of research on an already fatigued, ill body (Moss \& Dyck, 1999), dominated me and unsettled my confidence in two ways: producing anxiety that I would not be able to finish this project due to illness; and generating an urgent determination to see the project through. These conflicting needs 
slowed me down considerably, but also gave me time to reflect and continue. However, much of the service provided was only one aspect of the transcription task. Once I received the transcripts, I carefully and repeatedly listened to the recordings, going line by line to check for accuracy. With each interview duration averaging an hour, the fiftythree interviews took several months to cross check and analyze. Following this, transcript analysis consisted of coding for themes. Codes were first broken down into intellectual/theoretical themes, and organization specific themes. I identified overlap, repetition, and saturation. I then situated themes into groups from which I could make empirical/theoretical links and begin to construct the narrative presented here. Over time I went back to my original coding trees to weed out the concepts that would not be included in the dissertation due to being beyond the scope of answering the research questions. The refining of codes and themes seemed to be a revisionary process that persisted through analysis and writing.

The codes and themes developed during interview transcript analysis were then put to work on analyzing emails, web pages, photos taken during fieldwork, and notations concerning informal conversations. I examined an array of websites including all the KS local chapters, other sex culture websites, and sex-positive play parties and events in general. Differences between mainstream sex parties and KS events are often distinguished by many community members and there is evidence to these claims seen in media representations of other events. The comparison between mainstream sex parties and KS event materials is how some organizers and participants cite the difference. The difference in these visual representations speaks to what this community suggests inclusivity does or does not look like in terms of representation. Overall, the content 
analysis of emails, websites, and visual data helped to answer each of the empirical questions. And further provided evidence for supporting answers to each theoretical question.

Volunteer event production as observant participation, and becoming a participant

An important part of my fieldwork was the volunteer event producer phase (which overlapped somewhat with interviews). The field notes written and data collected during the second phase of research predominantly concern the production role, digging into what it takes to produce a KS event, and reflecting on the feeling of personal/professional entanglements with informants in the field. It was at this time that I became deeply involved in participant observation. At the beginning of data collection I met a KS community member who would become a strong ally and volunteer co-producer of KSNYC events. Shortly thereafter, together we formed a revamped KSNYC team and hosted our first event. When attending events as both a volunteer and guest, I was able to observe the deployment of play, community building, and performed sexuality. These observations helped me to explore the empirical questions concerning KS. For instance, answering the first question regarding the challenges of striving for inclusivity, was contingent upon being present, over time, in the scene. In these spaces I have been able to gain a firsthand account of contexts, behaviors, motivations, and interact with others in a manner that sheds light on the importance of a community imaginary. These observations demonstrate how identities are temporally shaped for both newbie participants and longterm members alike. Understanding how KS deploys care ethics and why such ethics are important for community members was also revealed during this extended time in the field engaging with the organization. In fact, it was not until I was able to observantly 
participate that it was revealed that care is important to community members and that such practices contribute to sex culture activism for many.

Toward the end of my time in the field, I stepped away from this volunteer producer role and shifted my focus to visiting Kinky Salons. In this final stage of fieldwork, I was able to visit eight KS events in total, five in the United States (San Francisco, New York, New Orleans, Portland, Los Angeles), and three that were established in Europe (London, Copenhagen, Berlin). The opportunity to do so helped me answer the third empirical question that asks: How does the case study of Kinky Salon disrupt or inform discourses of globalizing sexuality and sexual citizenship? Not surprisingly, the politics and economics of the event were revealed over time, as a result of more direct involvement with the event and community. However, participant observation in sexuality research came with a heavy set of personal and professional challenges (Comella \& Sender, 2013). Highlighting how this kind of work "raises questions of what it means to be a participant observer in highly sexual situations" (Comella \& Sender, 2013:2566). Over the course of my fieldwork it is this overarching statement, and its shifting meaning that I came to reflect on often in terms of who I am, what I am doing, and how that is affecting this project and those involved.

\section{Conclusion}

Returning to the two quotes at the beginning of this chapter, I highlight the overwhelming nature of sexuality research, that is often complicated by personal limitations and unexpected events. The representation of myself as both subject and object demonstrates the impact of intimate experiences and the influence of choices and decisions made as part of the methodological analysis and writing process. The pressure to complete a job 
well done is amplified by anxieties, which may only be relieved through an acknowledgement of self-care. As it turns out, the process of "processing" was a constant feature for me as a researcher while in the field and beyond. With regard to the complexity of experiencing emotions in the field, Laliberté \& Schurr (2015:75) argue: "Analyzing how certain identity categories become decisive or lose importance in the course of our fieldwork reveals how particular subject positions and intersectional encounters also frame the research process." These insights became instructive for me personally then, prompting me to reflect more deeply on my own process of relating to others in the field. However, aside from reflexively keeping a research diary, I have had the privilege of therapy, a good support system of academic advisors, friends, family, and community, which have helped immensely. Care can thus be viewed as integral to the pragmatic considerations of academic life.

In this chapter I have laid out the feminist methodologies used to answer the research questions that this study is built upon. I have also sought to understand the methodological challenges of doing critical feminist and embodied research in spaces marked by sexuality and taboo. I have done so by sharing some personal accounts that shaped my positionality in response to fieldwork, which also impacted how this project has taken shape. This project has made me reckon with how my sexuality, in the face of a health crisis, impacted my ability as a researcher in and outside the field. The inclusion here of field reflections and journal entries highlights my positionality and how it shifted or remained unstable over time, and the anxiety, insecurity, and uncertainty produced in the process. My healthcare process complicated this work. Learning how to create good boundaries and also achieve my data collection aims was certainly a lesson in self-care 
and good feminist care ethics. As a result, I have realized a certain sense of surrender in the process of prioritizing self-care that required I take more time to analyze and write up this text. Although I still struggle with the place of this personal journey within the academy, it has proven instrumental in processing emotions and my embodied place as a sexual being within the research process, academia, and life itself. All the same, what these reflections, insecurities, anxieties, and repositionings point to is a deeper need for an ethics of care - not only for ourselves as researchers, but also for a more robust engagement with our subjects.

${ }^{1}$ Heap, 2008; Hutchison \& Hutchison, 1995; Mumford, 1997; Nagel, 2003; Vogel, 2009, 2013; Watts, 2007; Wilson, 2010.

${ }^{2}$ For affect, emotion, and intimacy, see: Ahmed, 2004, 2014; Bondi, 2003; Davidson, Smith \& Bondi, 2012; Dixon \& Straughan, 2010; Pile, 2010; Povinelli, 2002; Thien, 2005; Valentine, 2008.

${ }^{3}$ For positionality and reflexivity, see: England, 1994; Faria \& Mollett, 2014; Kohl \& McCutcheon, 2014; Moss, Al-Hindi \& Kawabata, 2002; Wolf, 1993.

${ }^{4}$ For autoethnography, see: Crang \& Cook, 2007; Gorman-Murray, 2007; Newmahr, 2008.

${ }^{5}$ For insider/outsider relationships in the field, see: Browne, 2003; Cuomo \& Massaro, 2014; Taylor, 2011.

${ }^{6}$ For the embodied researcher, see: Bain \& Nash, 2006; Billo \& Hiemstra, 2013; Cupples, 2002; Ellingson, 2006; Longhurst, Ho \& Johnston, 2008; McDowell, 1992; Smith, 2014.

${ }^{7}$ For sexuality in research, see: Brown, Browne \& Lim, 2011; Cupples, 2002; Diprose, Thomas \& Rushton, 2013; Israel, 2002; Kaspar \& Landolt, 2014; Knopp, 1999.

${ }^{8}$ For care ethics, see: Atkinson, Lawson \& Wiles, 2011; Bondi, 2003; Green \& Lawson, 2011; Lawson, 2007, 2009; Massey, 2004.

${ }^{9}$ For globalization of sexuality, see also: Altman, 2002; Binnie, 2004; Gorman-Murray, 2006; Puar, Rushbrook \& Schein, 2003. 
${ }^{10}$ www.fetlife.com

${ }^{11}$ For the Internet as research method, see: Johnson, 2015; Karl, 2007; Madge, 2007; McGlotten, 2013; Neely, 2011; Phillips \& O’Riordan, 2007.

${ }^{12}$ For reflections on sexuality in fieldwork, see: Cupples, 2002; Diprose, Thomas and Rushton, 2013; though see Valentine, 2002; Bain and Nash, 2006 as exceptions.

${ }^{13}$ The take-away differences between them being the KS commitment to community building, education, and sexual revolution.

${ }^{14}$ A network of all the Kinky Salons established to date. The KSGC is organized to help facilitate cross cultural and transnational communications about the continual development and growth of KS, and how to be better stewards of each local chapter's community.

${ }^{15}$ Even though I received consent to use other identifiers such as a name or pseudonym, I made the choice to finally represent the interviewees in this way. 


\section{THE POLITICS OF PLAY: Negotiating power, connection, and transformation in a sexual community}

Kinky Salon founder: "I think a lot of sex parties are focused on sex, which still means that you're separating sex out of the rest of your social experience and making sex this separate kind of thing that you get weird about [...] because you're stripping out all of the community and the social aspect and just making it just about sex [...] What I want to do is normalize the experience so it's not really about sex; it's just about being a human being [...] I think that that is what separates out the sexual place of Kinky Salon from the other sex parties. I always called Kinky Salon a Freedom Party. Yeah, some people have sex - sure when they feel liberated but not everybody does all the time" (Interview by Zoe Margolis, recording by the author, KS Town Hall, London, March 2015).

"The politics of playing are primarily bound up in experiencing vitality" where " Play is a vehicle for becoming conscious of those things and relationships that we would otherwise enact or engage without thinking" (Woodyer, 2012: 318, 322).

\section{Introduction}

The sexual revolutions of the past have arguably evolved into the sexual social movements of today. In this chapter, I make the argument for play-based, communal sex as both political and worthy of our sustained attention within feminist and geographies of sexualities. Within popular culture, perspective shifts have broadened the field of choices from conservative ways of viewing the body and sexuality. Now, because attitudes around sex might more often be explored rather than repressed, there is an overall increased cultural visibility around once-taboo practices related to sex. ${ }^{1}$ With increased access via Internet technologies to a variety of sex-based culture, such fear has induced a more widespread sociocultural exploration of everything from stripping to pornography. Attendance and acceptance of strip clubs and the more prolific viewing of pornography through the Internet has helped highlight and normalize discussions of sexual cultures, albeit not necessarily in socially acceptable ways. Yet, what is so political about playful sex now? Here I investigate the claims that this event and community is different from 
other sex clubs and orgies. I then investigate the creation of momentary spaces as "safe containers," said to help facilitate a communal, playful engagement with sexuality. Showing also where ethical elisions lie within sex-positive culture reflected in this community.

\section{Intellectual setting and methods}

Scholars argue that the ludic is bound up with "an embodied, expressive, and affective immersion in the moment" (Edensor \& Bowdler, 2015:713). According to Woodyer (2012:320): "This is the motivation of play - the possibility of configuring alternate ways of being-in-the-world." In this case, the politics of play are entangled with the negotiation of power through connection. Sex-positive community building is an outcome of playbased sexual politics. These connections then pave the way for a community to emerge responding to the needs of its members. Weeks argues that a "critical community" is one that "results from a problematization of a given or latent identity...open to new experiences and ways of being, which make new subjectivities possible" (Weeks, 1996:73). These ideas dovetail with the objectives of play here as well, of creating new ways of being and relating to others. Further affirming the claim Cooper (2009:118) offers of "attentive sex play's power to affect," observed in the ways that participants speak about the good vibes, in sex-positive language, tending to gush about why they love sex culture so much. Communitas foregrounds such connectedness as "social insideness," although it also asks us to consider what the "vibe" means to participants and how such an intangible geography is spatialized (Twine \& Gardener, 2013). Generating a vibe through the creation of space is also about subject-making: "The subjects of communitas that are so profoundly connected - are so constituted - because they have 
plugged into the same affective currents by means of their participation in a particular form of life" (Twine \& Gardener, 2013:51). Connection then, is an affective experience only accessible to those who are privileged to enter, be at home, and feel welcome in such spaces communitas thus takes place. Such intentional curation of space through the affect of vibe co-consitutes the way places may be collectively engaged with (Twine \& Gardener, 2013). This culminates for example, at the point of reciting the Kinky Salon Pledge together as I discuss below. It is therefore also important to understand how subjectification is achieved through such spatial practices. How sex party-goers become subjects is reproduced in the languages and expected behaviors of those who are welcomed into the space, also foreshadowing the "affective potentialities of places" (Twine \& Gardener, 2013:57); and the limitations of inclusion through such subjectification.

Some of these limitations are realized through the policing of play that emphasizes safety. Safety is thought to be achieved through surveillance, safe sex practices, and the practice of affirmative consent. Nevertheless, consent as a concept and praxis is contentiously defined. The culture of BDSM predates and sets a foundation for consent for this case. S/M communities that have preceded communities like Kinky Salon, have established and codified certain cultural functions to: “(1) demarcate boundaries, (2) provide a story of origin, (3) establish codes of behavior, (4) create a system of shared meanings, (5) provide a means of social reproduction and (6) generate sexual identity" (Downing, Langridge \& Barker, 2007:25). This is important to underscore, since consent is a fundamental cornerstone of alternative sex practices such as kink and BDSM, and any kind of edge play (Weiss, 2011) - where a participant is 
tested and brought to an edge of their comfort zone, which may include what some consider "harm to self." Trust is necessary and implicit for healthy, consensual scenes to play out. Recent scholarship reflects that "the ethics and erotics of sexual consent" is no longer as taboo a subject as it once was (Leisenring, 2012); that a 'just say no' approach is problematic and contradictory (Burkett \& Hamilton, 2012); and that responsibility of gaining consent is shifting from individuals to communities, as the concept begins to focus on power dynamics beyond the sexual (Barker, 2013). Beres suggests: "talking about sex with a prospective partner is often considered taboo," and asks, "what is sexual consent, and how is it defined?" (2007:94). In BDSM communities, the precursors to this particular community, it has been revealed that "pleasure works in concert with the rules to produce SM subjects" (Weiss, 2011:100).

Nevertheless, in social, legal, political, and sexual spheres, there are discrepancies about what constitutes consent, how it is given, how it is interpreted, and what happens when misinterpretations occur (what to do about fault and how to move forward from violations - are they intentional or unintentional?). Despite emphasizing a clear yes before sex, this version of consent is regarded as incomplete. The urge is to orient consent culture to a version of sexual consent that recognizes the challenges of being situated in a larger culture that is considered non-consensual (Barker, Gill \& Harvey, 2018). According to Meg Barker (2013:896), prevailing norms place the responsibility of consent "with the individual (historically women) and consent relates to sex rather than the relationship as a whole." This is extended further in what other scholars contend is needed: "a deliberate version of consent $[\ldots]$ referred to as communicative sexuality" (Pineau, 1989; Beres, 2007:102). This suggests that individuals have a dialogue to 
communicate their willingness. Such nuanced difference reinforces a broader view of consent as holding communities, rather than just individuals, accountable. Within sexpositive communities, how this is reconciled at play parties is that participants are assumed to know, or are quickly indoctrinated into speaking about consent and boundaries. However, the ideal and reality are sometimes in conflict. In the aforementioned communities, enthusiastic consent is encouraged as the foremost safe way for individuals to engage appropriately. Consent as a "culture," and the tagline "consent is sexy," is quickly becoming the commonplace language of alternative sex communities. For many, consent is understood as necessary for daily life, outside the sexual. However, consent violations happen, somewhat in part due to how consent is variably spoken about and understood.

Despite the burgeoning scholarship on alternative sexual practices and sexpositive community, there are gaps that need to be addressed. Firstly, revolutionary language and activism, historically has obscured privilege and racial aggressions that serve to reinforce rather than subvert the status quo. Subcultures of the past have often reinforced mainstream oppressions (Haenfler, 2004) through tropes of colorblindness and attribution error (Bonilla-Silva, 2017; Doane \& Bonilla-Silva, 2003; Levy, 2018).

However, this case takes a much closer look at how liberal privilege works within sexpositive culture and community, given the reproduction of liberatory agendas.

I respond to this opportunity to focus on the politics of play and negotiations of power at a sex party that aims to change the world with its deep hedonism. ${ }^{2}$ I interrogate the effectiveness of ethical hedonism in the service of what I term a softcore ${ }^{3}$ sexuality activism, which may or may not restructure the so-called neosexual turn. To do this, I 
analyze how inclusivity and space are mutually constituted and at the same time, embedded with the tensions inherent in trying to serve the needs of a diversifying population who want to be involved in sex-positive community and play parties. However, bodily proximity is not always necessary, and through the Internet and social media, impressions and collective feelings may develop where the screen is the surrogate for skin (Ahmed, 2004). Finding that, play-based intimacies are said to contribute to experiences of community, often described by participants as transformative. Therefore, in this chapter I answer the following research questions:

Theoretical question: What is so political about sex play, or playful sex? Empirical question: What are the challenges inherent in striving for inclusivity in sex-positive community? Does Kinky Salon's vision of playful sexual revolution create change? For whom and how?

I explore these questions using autoethnographic accounts and interviews that highlight how this organization and community understand the politics and power of play. Through participant observation, interviews and informal conversations, I investigate how sex-positive politics aim to rework sex through play, by reframing play as political and power laden. Such conversations reveal both the promise and peril of being invested in a sexual community. Participants claim that play is transformative, so I explore how and why these claims are made through interview data and online resources that demonstrate what participants claim sets this community apart. I explore websites and apply to so-called mainstream events to find out how a KS politics of sexual play stands in opposition to these other spaces, and how those politics operate. Finally, the potential for transformation and change is not without its challenges, however. I analyze 
the efforts to identify and mediate power imbalances, observing who is involved (or not) and why. This underscores how class and racial sociocultural politics are understood or challenged by the actors reproducing the KS ethos. A sexual politics of play then, is subject to the tensions inherent in the remediation of power that seeks to amend the uneven process of promoting diversity. I reveal what happens when play isn't liberatory for all by highlighting the costs associated with striving for inclusivity.

\section{Establishing the playground rules: What is so political about playful sex?}

Many participants I interviewed claimed it is a liberating experience to be able to experiment with alternative ways of being and relating to others. Sexual politics enacted through play and connectedness encapsulates the KS community vision of making the world a better place for people to freely express their sexuality. For many community members, communal sexual play becomes an intentional way of changing lived experience, deconstructing dominant (and some say, oppressive) norms, values, laws, and structures that limit autonomous, embodied expression. The importance of play for KS community members is likewise attached to the idea that "playing's self-affirmation can stimulate a generosity of spirit toward others... This is achieved by our being affected. In this sense, the proximity of play is self-perpetuating as the vitality emerging from it encourages one to be more responsive to others" (Woodyer, 212:319).

An ambiance all fun and play. Heading out into the night I'm dressed as a Candyland board game character. This isn't any ordinary costume party; this is a Kinky Salon "play" party. I arrive and walk into an unmarked venue. As I enter, narrow hallways open up into rooms sparkling with colored lights and glittery décor themed for different types of encounters. Some rooms are filled with 
comfortable sofas ideal for chatting and getting to know other partygoers. Other rooms are designed specifically for sexual encounters, with cloth-covered beds, sexual apparatus, and safer sex supplies strategically placed on surrounding surfaces. The evening begins with theme-specific music, loosening up on the dance floor, and an exploration of the spaces created for the night. As the night progresses clothes come off and partially clothed and naked people are seen everywhere, even on the dance floor. There's a midnight cabaret and performers mingle with guests to create a gregarious party feel...

Participants overwhelmingly reproduce the rhetoric of playful ideals that are at the center of the KS mission that seeks to reimagine a contemporary sexual revolution. As one example, while I was in the field what became quickly apparent was how often I was saying the word "play." It actually got on my nerves in the beginning, I imagine the same way overusing the word "love" or "awesome" feels. The frequent tag of "play" in this scene was ubiquitous:

Reflections on the concept, play, in the sex-positive, sex party, BDSM worlds: Everything is related to the act of playing. Play party, play partner, edge play, piercing play, blood play, safe play, unsafe play, play with, play room, play space, play scene, play (a) character, play time, fetish play/dungeon play, group play, play free zone/no play space, sex play, play (party) community, power play, BDSM play, playground...

Woodyer suggests that there are playful residues in adults and those "residues have the potential to spring forth as revolutionary consciousness, opening possibility for more willful social transformations" (Woodyer, 2012:318). For instance, a recent flyer 
for a KSSF event, "Cybersex" reads: The future is now. Tele-dildonics and live streaming scat chat. VR sensuality and Google glass porn. Hardwired into the mainframe for Sex 2.0, broadcasting in Bluetooth and tethered to your wifi enabled fleshlight for a night of anonymous ass hacking! Between double entendres and cheeky puns, the language evident in KS materials aims for devil-may-care chuckles layered with sharp imagery and pop culture references. This potentially offensive, in your face, silly attitude permeates community and event representations. I argue that this is one of the ways in which the sex-positive ethos instills an ethics of resistance into the community in a manner that may be palatable, or relatable to even the least political of members. In other words, this type of play, to do with sexual play, even whilst outside the act of sex itself is strategic on the part of KS: "Positioned as a counterpoint to the conventional, playing is couched in a framework of resistance. This is reinforced by studies that frame playing as resistance to adult culture" (Woodyer, 2012:316). The language, themes, and costumes serving this purpose well.

For many participants I spoke with however, they want their fun, playful sex without the politics. Be that as it may, just because one does not think of themself as a political actor does not necessarily make that the case. On its own, the sexual play and community events produced by KS also reproduce participants as political subjects simply for subscribing to the Charter (i.e. behavioral agreements) at the door, if you will. This sex play is spatial. This sex play is always already political. For these participants and community members the rewards are deep hedonism, and being held in community, which is often perceived through rose-colored glasses. And at the same time, play is what 
makes sexual politics accessible, because there is something in it for anyone who dares to try it out. For instance:

\section{KS participant (30s, fluid heterosexual, male, Caucasian, creative}

professional): “Yeah. I think there's an innocence that can be experienced through learning how to play. Something that a lot of citizens have lost. It's a chance for escape from our day-to-day responsibilities and a lot of us are in a position where you have to be a certain... We have to be a certain way because people expect us to be that way. And in order to I think become more whole, become a whole natural person we need to be able to put that aside. And that's the whole idea with costumes is to wear different hats for different situations" (Interview, February 2015).

This point is made clearer through my own observations during one of the first events I attended, where people play with their identity presentations: What I saw at this particular party were a panoply of sexual energies and identities: manifest, latent, identity affirming or creating, or simply an essence, a flair, the way a body moves through space relating to others' sexuality performances, polyamory, fluid sexualities, gender neutrality or androgyny, hyperfemininity, and the occasional overt, hetero-styled gender performances were the most prevalent in this space. The KS manifesto, illuminated by the Pledge further highlights the integral nature of humor and play for the community (excerpt from the KS Companion Manual):

Emcee: Place your right hand on your heart. (Pause while the audience puts their hands on their hearts) 
Emcee: Place your left hand on your neighbor's buttock. (This always gets a giggle. Remind them to ask first, and give them the option to offer another body part)

Emcee: Now repeat after me: In Pervitude and Servitude

Audience: In Pervitude and Servitude

Emcee: United by our dubious morals

Audience: United by our dubious morals

Emcee: Well dressed and ready for action

Audience: Well dressed and ready for action

Emcee: Call us perverts for we are proud (punch your fist in the air)

Audience: Call us perverts for we are proud!

This collective action is translated into one of the ways organizers focus playful actions to break the ice at a sex party. Demonstrated throughout the space of the event, particularly coalesced around the moment the pledge is recited are moments of affectual investment, said to open people up to experiment with vulnerability, sharing, and new ways of being and relating to each other:

Everyone was into it. The audience raised their fists into the air, got/gave consent to place their hand on their neighbors butt (rather than over the heart) and recited the Pledge of Allegiance. It was silly and rhythmic, with laughter, shouts, and fists pumping high in the air.

These moments begin to illuminate how play is fundamentally subversive. Rahier (2013) argues that adult plays suggest we can manipulate our perception and relative actions, with regard to the way things actually are. "In that sense, plays have a subversive 
potential because of their power to evoke how things could be, or how things could have been" (Rahier, 2013:5). Subversive play evidenced in these collective behaviors then, draws people together, blurring the boundaries between people that may be present outside the KS created space. Nicholls (2009) argues that such interaction "weakens the boundaries between self and other, making it possible for people to 'discover' common interests and values across traditional cultural and sectoral divides" (2009:84). To do this, KS organizers intentionally create a certain type of sexual public where "collective action made to realizing and sustaining this public" is reinforced with a "sexual power [that] is centrally situated at this interface" (Cooper, 2009:116; see also ${ }^{4}$ ). This appears to be how KS events operate and at the heart of play for (most) KS participants is the theme and attendant costuming for each event. This aspect is an essential part of the "magic sauce" of what makes Kinky Salon stand apart for many. Yet, it has also been an aspect that has been a barrier to entry for others. For people uncomfortable with what it takes to put a costume together and put it on, it can be a deterrent to participating. Such "affective assemblages" like décor, costumes, and behavior, are established to maintain cohesion and acceptable practices that establish how in/exclusion may be interpreted (Twine \& Gardener, 2013:67). However, there is a culture constructed within the space of community that those who buy into this collective behavioral agreement, bond over.

KSSF participant, volunteer, and cabaret performer (40s, bisexual/poly, female, white, massage therapist and educator): "I feel like the sense of play is really important [...] in a way that allows for a kind of playfulness or goofiness [...] Like you don't have to be wearing like a $\$ 300$ fetish outfit [...] if somebody wears something clever it's like A for effort. If somebody wears something that like isn't 
clever but they just feel sexy and comfortable in their body it's like A for effort..." (Interview, February 2015).

The Kinky Salon cabaret is another moment of collective play that is an event within an event. The cabaret speaks to the type of adult play that has been theorized as a tableau of reality. According to Turner (1979:494), "subversion often takes the form of rational critique of the established order" but can also take the form of the irrational, humorous, or absurd. It is a moment that points to an intentionally carefree, yet thoughtful orchestration of time and attention, a focal point with a message: artful anarchy, irreverent commentary, physical display. Ahmed (2007:152) argues that, "What you come into contact with is shaped by what you do: bodies are orientated when they are occupied in time and space." Thus, the marking and transforming of time becomes spatialized in this way. As such, directing time and attention is a feature of how power operates. With the pledge and cabaret being emblematic of this time away from time, anticipation and expectations are also managed.

The midnight cabaret captivated the audience with the silliest burlesque, circus and lounge singer acts. Certainly not a serious show, the talent was equally sexy and absurd, with one boylesque performer dressed as a chicken and as a finale pulling a live chicken from his go-go shorts.

In these ways, Kinky Salon events create such liminal space for an evening, where a moment is available for a collective, shared experience that feeds back into the architecture of the community. The cabaret is this moment between arriving and the possibility of sex, a redirection of intention to collectively witness each other: 
KSL participant and volunteer (40s, bisexual, female, white, journalist): "So humor is really important and so the Cabaret is really important, actually they go hand in hand, it's never this deeply serious thing. It's always very playful-very funny. (KS Town Hall London, recording by the author, March 2015).

Yet, does this intended effect hit the mark for attendees? In other words, is humor that serious? Although these performances may well provide some sort of momentary catharsis, they more often end up reinforcing the sociopolitical order of daily life (Rahier, 2013). In this case, it is the reaffirming of togetherness, although without the critical unpacking of what that togetherness means, or the costs it could entail.

KS founder: "The cabaret is really about having a moment to be together. It's really not about entertainment at all. That's just an excuse to get everybody sitting in the same place for a minute so that we can go "Hello, welcome!" and have a moment to be together [...] we realized that when we have a cabaret it really changes the way that people interact. The cabaret is the time when everyone goes [...] "Oh yeah right we can just be ourselves here. Cool" [...] and it's also a place to showcase the talent of the community" (Interview by Zoe Margolis, KS Town Hall London, recording by the author, March 2015).

Silly, collective moments like the cabaret bring people together to hold space for each other and witness the absurd entertainment. The cabaret reinvests participants and the space itself with the sardonic character of the theme for the night. This moment reinscribes the importance of play through the acts, reflecting back to the audience in an interactive manner that the intersection of play and sex should not to be taken too seriously. Poking fun at taboo, all the while holding space for non-conformity, acts to 
facilitate opportunities for what many in the community call "heart-centered relating" through sexual expression and play bonding.

What helps establish the rules of play further for community members, and other sex-positive communities, is the enactment of sex-positivity. Sex-positivity and nonmonogamies both contribute to and complicate power relations within the community. These concepts are fundamental to understanding the worldviews of participants, getting at how and why they construct play as transformative. I therefore dig into the claim that by building a culture of acceptance, play can be engaged with more easily. For instance, a Facebook group allied with Kinky Salon called Trustable Sluts, defines sex-positivity as: "Celebrating and embracing open sexuality with few limits, beyond an emphasis on safe sex and the importance of informed consent. This includes welcoming those whose sexual appetite might not only be the same, but perhaps different from yours." It is a claim that is full with a litany of assumed ways of communicating and behaving. As the literature points out in the Introduction, there are many ways to conceptualize this, as well versions that are subject to constant interpersonal revision. But, sex-positivity remains problematic. Notions of what sex-positivity means may distill the most utopian ideals down to essentialized, potentially violence-laden discourse:

Wider play party community member (45, straight, male, white, BM sex-party producer): We feel that sex positive is obviously redundant and... We hate that term. What is sex negative? So we believe that all people are sexual. And you're either going to admit that or you're a liar" (Interview, February 2015).

As I uncover the contentious ways sex-positivity is employed as a framework for sexculture, how privilege works demonstrates the ways positivity tropes are differently 
rendered. Nevertheless, for sexual communities such as Kinky Salon, the concept "sexpositivity" is a defining feature, with the presumption of acquired knowledge and skills necessary to embody the concept:

KS attendee (30s, queer/poly, female, Caucasian, business operations manager) affirms: "if you can't talk about something, you're not at a level where you're ready to enter a world like that. If you're interested in talking about it, come on in, but if you're like no, no, no, I don't want to talk about those things... if you're not willing to say what's on your mind, we're going to have many other problems and that's what I mean by sex-positive, like, being willing to talk about it" (Interview, February 2015).

Foremost among those thinking about sex-positivity is the claim that much of conventional norms and attitudes around sex are negative and rooted in shame. The KS organization and community members claim that such conventional, sex-negative social discourses dominate society such that sex and shame are entwined. These assertions hearken to the claims made for liberation during the sexual revolution, suggesting that people still feel stigmatized by sexual desire (Allyn, 2016). My discussions with respondents often reflected on common bonding experiences over sexual shame. And how the KS, sex-positive community mediates this through connection, empathy and support, is firstly organized through fun. Adding complexity to the question of sexpositivity, what is it, and who gets to define it, is what happens when personal and social expectations are restructured around this shifting concept. Yet, while some fetishes or D/s (dominant/submissive, edge/role play) relationships engage with consensual harm of self 
and other, the question then becomes what makes something sex-negative or -positive, and who gets to make that judgment?

KS attendee and wider sex-positive community member (30s, straightish, male, black identified, IT marketing and sex-positive play party producer): "sexpositive basically means, to me, like accepting and supporting everyone's right to participate and become sensual, sexual behavior that turns them on [...] that they choose for themselves. I know that there's some conversation right now [...] about whether we should be sort of pushing sex-positivity as a frame versus something more like sex neutrality as a frame [...] that it's a more of like a study on privacybased argument. It's more like the right to be left alone [...] And then the other piece of it, of course, is that, for many people, sex isn't a particularly positive thing [...] for people who have maybe sexual traumas for instance or people who are asexual, do they have to fully support your thing? Or is it enough for them to, you know, you're okay with you doing it, sort of outside of their view" (Interview, March 2015).

Alternative relationships are similarly highlighted as one of the grounds upon which the sex-positive praxis of this community is applied. This is especially important for those who have so-called taboo or deviant lifestyles - in terms of societal norms. Observing that: People who practice non-monogamy or polyamory as lifestyles, organize themselves around their sex-positive social events. Community is invariably organized in and with events such as play parties, and weekend getaways with play spaces and workshops. And, respondents were very particular about how they self-identified along these lines, specifically clarifying the role intimacy plays. For instance: 
Wider sex-positive community member, KS adjacent (20s, queer/poly, transgender/nonbinary, white, lawyer and activist): "I've been thinking a lot about non-sexual polyamory because a lot of queers, especially, people like in the “sex-positive” community are really good at separating sex from let's say emotional intimacy. And I don't say that with judgment I think recreational sex is a simple form of activity. But the separation of those two things for a lot of people it doesn't always extend to a real deep interest or investment in non-sexual love between peers. And so, when I think about negotiating intimacy, you know, having the physical act to sex is certainly a cool thing and certainly a thing that people who are intimate can do to manifest that intimacy. But I don't think that sex is necessarily a prerequisite for intimacy or a requirement" (Interview, February 2015).

Yet, there are other community members that reservedly take some distance from being overly sex-positive, suggesting that, like anything, there are pros and cons, positive and negative sides:

\section{KS attendee and volunteer (40s, queer, female, Caucasian, sex worker}

consultant): "it seems like the way people are kind of quantifying poly is the ability to have ongoing sexual and romantic relationships with people. I think that sex-positivity and polyamory are not mutually exclusive but there can be poly communities that are sex negative” (Interview, January 2015).

This is further reflected in this respondent's sentiments suggesting a strong dose of “alternormative"6 (Cooper, 2007, 2009) reality: 


\section{Wider sex-positive community member, KS adjacent (20s, queer/poly,}

transgender/nonbinary, white, lawyer and activist): "sexual freedom means different things to people with different levels of power. And when you're a straight dude, sexual freedom means the freedom to have whatever sex you want to have without repercussion, whereas when you're a queer person or a changed person or a person of color or a woman at all, sexual freedom means a lot more like the ability to have sexuality without incurring violence for it, right. And so I think this also informs the difference between sex space that straight men make and sex space that the rest of us make, because when a straight man makes a sex space, his goal is I'm going to make this space for me and all my bros to get laid. That's what I want” (Interview, February 2015).

This reality is reflected in the earlier quote that denies the existence of sex-negative behavior and the power of privilege invested therein. These contentious realities inform many participants' daily experiences. All of the members and participants of the KS community are not exclusively non-monogamous. However, many are, or are very fluid in their relationship practices such that they are continually revised over time. And since there is an assumption of being sex-positive in the community, the ethics that are central for practitioners of CNMs also overlap and feedback into vernacular knowledges (Escoffier, 1998) and agreements that drive the sex-positive cultural community standard. Community members then find additional support within a communal space that values alternative relationship structures through the development of alternormative constructs of mutual understanding. 


\section{KS attendee (30s, queer/poly, female, Caucasian, business operations}

manager): "Polyamory plays a role in my life because I'm in a community that knows the word... I feel like one foundation of our relationship is our community" (Interview, February 2015).

Former KSNOLA co-producer, volunteer, and participant (20s straight/poly, male, landlord/performing artist/teacher): "one of the benefits that exists to that label is it gives a rally point for community, there's a support network... you can access a lot of information, you can access a lot of other poly people [...] it can be really frustrating and confusing and finding other people that are doing it similarly can be very healthy and inspiring [...] When I'm looking for answers to questions and I'm looking for resources I know where I can look" (Interview, February 2015).

Once this basic language of sex culture has been adopted and understood, participants are more likely to find it easier to relate to others within a sexual community. This all deeply informs how participants navigate sex-positive community and events, opening up to the potential of playful sex, and the importance placed upon such potentials to change sexual norms.

\section{Negotiating power and connection: The policing of play}

In order to understand the entanglements of play and power, I investigate how people become "enmeshed in webs of power" (Bailey, 2002:265). For the KS organization, the intent to deconstruct power imbalances can be seen in a number of its formulaic structures. The PAL system for instance, or Pervy Activity Liaison, is a system that was designed to help police behavior among participants and is claimed to decentralize 
authority, placing responsibility for consensual behavior directly with the participants. Everyone who attends Kinky Salon must be accompanied by no more than two PALs (pairs or triads). These are people that one knows and can vouch for if problems should arise. PALs do not need to be lovers or partners; they can be friends, people of the same or different gender, or sexual orientation. This structure is considered a function of “distributed accountability" to have pairs or triads be responsible for each other's behavior:

KS founder: "The Pal System basically means that [...] you are responsible for that person's behavior for the night and they are responsible for you [...] we're all responsible for one other person. It means the problems are solved before they even start [...] because people want to take responsibility and know that their behavior is going to be reflected on their PALs" (Interview by Zoe Margolis, recording by the author, KS Town Hall London, March 2015).

If problems do arise, PALs are said to be taken aside together to check in and mediate issues. For instance, if someone is getting too intoxicated, their PAL is alerted and made to help. If someone is accused of any transgressions of consent or otherwise inappropriate behavior, both PALs may be asked to leave, or in extreme cases, be banned from future events. In this way, it is thought that the PAL system makes individuals look after each other and keep each other in check, since no one wants to have their evening interrupted by a PAL that is not following the rules. The objective pleasure received by ascribing to rules also reaffirms subcultural norms for participants. Certainly the PAL system is not foolproof, nor is it without its problems. Namely among them is the barrier to entry that 
this system may pose for people new to the community. It could also potentially miss the problems inherent in a "bad apple," where the PAL may be no better. ${ }^{7}$

Another fundamental structural element built into the KS formula to mediate power and police behavior, is the Charter. ${ }^{8}$ The Charter was created by the community, and is a comprehensive list of guidelines to abide by in the event space. Some KS chapters add new rules given their particular community's needs. The Charter is an agreement about appropriate behavior that contributes to the politics of building a "safe container." 9 Participants are made to read and agree to these behavioral mandates before entering the space. Such “affective routines" are reproduced by participants' willingness to play a role (Twine \& Gardener, 2013:53). In order to recreate cultural norms in this direction, for example, an ad for a KS sponsored workshop reads: Play Party for Beginners. A low-intensity, low-pressure, intimate play party for people who are just starting their play party life! Come and hang out, meet people, play consent games and maybe even play a little!

The practice of affirmative consent is demonstrated in the above Charter, and is a cornerstone of KS sexual ethics for community members. Education tools such as the Charter are implemented with the help of event hosts and volunteers at the entry and throughout each Kinky Salon from the moment one enters the space. Workshops on enthusiastic consent are held at Burning Man and in sex-positive play party communities. Consent has become part of the Comic Con culture, where touching cosplayers ${ }^{10}$ without consent became a primary concern prompting visible signage at events articulating what constitutes consent. And it is the subject of a number of mainstream popular culture articles geared at young people. ${ }^{11}$ Thus, the importance placed on consent is reflected 
upon walking in the door, as guests have a very frank but fun, clear conversation about the boundaries encompassed in the Charter. An emphasis on clearly stating boundaries opens people up to talk more directly about sex.

Curating such a space to promote and police sex-play is something organizers take great care in doing both online and offline. Managing space is centrally important to providing opportunities that allow people to feel comfortable to become vulnerable. For instance:

As a walkaround host, my job was to greet guests; show them the layout of the venue with its different spaces for different activities like no-play, sex play, BDSM play, and dancing; check in on party-goers throughout the night; maintain supplies and ambience; and be available for questions. It was more or less a position to thoughtfully mingle.

Once coming through the door and being greeted with behavioral agreements in the Charter, guests are ushered into the venue and introduced to their temporary otherworldly environment:

The first time I experienced Kinky Salon I volunteered as a walkaround host for the 11th Anniversary party in San Francisco. Before the event, I helped set up the space, which was an artful practice of setting the scene: we created partitions for different rooms; prepared the spaces with multi-colored lights positioned just right; fluffed cushions everywhere; arranged mattresses with linens; hung theme specific décor from walls and entryways; and placed safer sex supplies on every flat surface throughout all the venue's rooms. 
Often the act of setting the scene, as demonstrated above, requires local KS events use inventive and creative manipulation to turn the venue into another world (i.e. good ambiance, multiple rooms, dance floor, space for a DJ, coat check and bar, and space for FUNgeon ${ }^{12}$ equipment, with theme specific décor and plenty of seating, etc.). At KS events this is done with props and music, lighting techniques, whimsical use of draped fabrics, and strategic arrangement of furniture. It is important to stage the venue with different areas for people to explore, play, or just hang out. Doing so facilitates a "ludic engagement with space" (Edensor \& Bowdler, 2015:723), suggesting that spaces are engaged with by participants in ways that are unscripted, and with frivolity and playfulness. A space can be thus created through the imagining of ludic potentialities, embodying the movement and action of "going with the flow" (Edensor \& Bowdler; Turner, 1979; Woodyer, 2012), creating space that just "feels" right.

KSNYC co-producer, volunteer, cabaret emcee (30s, bisexual, female, white, hairstylist) echoes these sentiments: "I love the interactive games and stuff that we come up with and the cabaret because I think that that really does set it apart and the fact that it's a themed club [...] Kinky Salon has a new theme every time and it makes it fresh and it makes it new. And it's almost like, even though, the space may be the same it's going to be a whole different event because the tone of the event is completely different and everyone is in different costumes" (Interview, January 2015).

These staged areas affect a vibe, but are also created to corral different activities into different spaces in which guests can be monitored or contained. Such efforts may therefore unintentionally complicate liberatory values via the discipline of collective 
surveillance of one another, and the management of power according to a certain flow. In this way, the vibe can also be experienced as exclusionary, where "the effects of power in terms of privilege, marginalization, oppression, etc. - become apparent in the vibe as it animates bodies, channeling conduct along trajectories that collectively bring privilege into being" (Twine \& Gardener, 2013:66). Not feeling like part of the group, not partaking in group activities, and not feeling "chosen" are obscured but real experiences of the space.

The presence of drugs was not initially observed, but as the evening went on, it became more apparent. There was not an overwhelming reading of the space as one that was heavily influenced by drug use, i.e. people were maintaining a moderate level of decorum not acting in ways that would appear under the influence, not at least any more than the basic tipsy intoxication. I move between rooms to... just keep moving... This playspace is filled, nowhere to move, but no one cares. Talk and laughter drowning out the music, and the smell of sweaty mixed up bodies in states of carefree nakedness. I need to get out of here I feel claustrophobic.

In these ways too, difference is clearly marked between everyday social space and liminal space because "performances require framed spaces set off from the routine world" (Turner 1979:467). Producers clearly design spaces that set the event apart from the ordinary world, and often cannot be tracked by ordinary time. This further demonstrates the point made earlier regarding how the co-constitution of time and space is also about curating opportunities to deconstruct cultural norms and behaviors. For a moment, KS 
events are transformed, "configuring imaginative, miniature, virtual and affective spaces" (Woodyer, 2012:320) to facilitate ludic experiences via playfully sexual encounters. Former KSNOLA co-producer, volunteer, and participant (20s straight/poly, male, landlord/performing artist/teacher): “We don't say that you can't fuck on the couch but we definitely cater different parts of the physical venue towards different energies. We try and have some quiet relaxing areas, some higher energy party areas, and some areas that are clearly designed for sexual connection in a very physical way" (Interview, February 2015).

These processes highlight how a politics and policing of play is articulated and lays the foundation for the ways in which intimacy and connection are made possible for participants spatially. This suggests that play and leisure are contingent upon this approach that I will argue in chapter four, is care-focused. In any case, it was often remarked that the curation of space is what helps to make the night enjoyable:

KS volunteer and participant (30s bisexual/poly, female, Caucasian, health care worker): "I think it really depends on the space and the people. The setup of the space where it is to happen and where the sex party happens but also, for lack of a better term because it sounds like super hippy dippy, the energy and the connection between people. But I think the space set up has a lot to do with it" (Interview, February 2015).

In addition to creating playspaces (or what organizers refer to as "horizontal socializing space”) with beds, linens, cushions, and low light, it is strongly suggested that there be at least one area where people are not having sex, the "no-playspace." This nuanced act of creating alternative spaces was an arrangement conceived of in group 
meetings with organizers and community members. This refinement of space is claimed to ensure that sex is not the focus although it is happening. And further used to signal places where power can be mediated away from negotiations of sex, desexualizing interactions. Informed in part by Turner's conceptualizations of communitas and liminality $(1979,1982,1988$; see also Bigger, 2009), I argue that the rationale grounding this act demonstrates the play and care-focused politics I claim are most relevant to the neo revolutionary Kinky Salon sex-positive agenda. Functionally, this move potentially helps to make the scene more comfortable to newcomers and anyone else enjoying the party in non-sexual ways. It also suggests a commitment to reinforcing the spatial construction of inclusion. Diverse areas help facilitate communitas where people are “'just as they are,' getting through to each other" (Turner, 1979:471).

Getting through to each other happens outside the space of events as well. The online presence of KS has helped to further establish connection beyond the scope of events and is an essential component of mediating playful intimacies for participants. Social media outreach and networking are vitally important to the development and growth of the community. The founder states that without the Internet and social networking, Kinky Salon could not have proliferated: "Without it, you have to have private parties. With it, you don't have to be private because you can invite your friend's friends $[\ldots]$ now, you can have an event that's available on social media and it means that your friend's friends will see it. So it's not private, but it's not public" (Interview, August 2015). Being publicly private (Lange, 2007) enables KS organizers to manage the privacy for each event without having to publicize to a wider audience that might not ascribe to the sex-positive KS ethos. For organizers, the safety of the container is important because 
of the level of vulnerability people may feel simply being at a party where sex is also taking place. This is one of the ways in which participants are made to feel safe in the space, both online and offline. The ability to remain publicly private allows participants to reveal their identities to others within a private network, with content remaining relatively private because it is not widely accessed outside the network (Lange, 2007:361). The creation of a safe container is said to enable playful connection and intimacy unfold:

KS volunteer and participant (30s, heterosexual, male, African American, selfemployed creative): "There's a sense of safety like, to the utmost. And how the Charter is presented and how the whole Kinky Salon structure works, I think, it has an underlying sense of kind of safety, trust, like awareness that I haven't seen in some of the other events. And I think this idea of you're responsible for the person you come with, and if everyone has that idea, then things work out really well” (Interview, January 2015).

The strong emphasis on safety and focus on creativity is a key part of creating social change based on play that is in turn viewed as transformative by participants.

\section{The transformative potentials of sex-positive community and playful sex}

Being set apart from mainstream sex parties is an important point to make for the participants of this community. Commonly labeled a sex party, KS organizers also take special care to distance the community and events from mainstream sex parties. One way that this play party is distinguished from mainstream sex parties is in part by focusing on accessibility and inclusivity. 
KSL co-producer, volunteer, and participant (30s heteroflexible/bisexual/poly, female, mixed race, sexual health outreach advisor): "It's not an orgy. It's not an orgy. There's people who are consenting in their particular pods or groups or whatever it is and it's not this free for all you can touch and do whatever you kind of want, it's not an orgy. There's no way that I can present it in a way that people think that they can understand it because it's not that. They have to go on the other hand, oh yeah there's 100 people having sex but it's not an orgy" (Interview, March 2015).

The difference for this community is not about pleasure for pleasure's sake, as that can be constructed as radical too. The difference is rooted in playfully coming together in a creative, sex-positive community, to safely experience sexual pleasure.

Former KSPDX co-producer, volunteer, and participant, (40s, bisexual/poly, female, mixed race, social worker): "The creativity. I feel like it really tries to be creative and I feel like they really want to have fun. I feel like they really like doing the creative side, having to be in costume and stuff I think it drives in a different crowd than say would be at [...] a random sex party somewhere, you know? I feel like there's also a really conscious effort for safer sex supplies being out which I really like. That I feel there's a lot of talk about, how do we make the party bigger and better every time. I like that openness about it, but the creativity is really my favorite and I think that's really one of the big things that makes it different" (Interview, January 2015).

Perhaps a small distinction to the inexperienced observer, this is not the case for KS participants. 
Although the comparison with mainstream events is not a central focus here, it provides texture to the narrative overwhelmingly reproduced by community members: that Kinky Salon is different and that difference sets up the potential to envision personal and social transformation. The verification of such claims with regard to the actual transformational potential that may be found in mainstream sex parties, is outside the scope of this project. However, there is more here to understand. It is assumed by participants that mainstream sex parties are not sex-positive (i.e. sex-negative), reproduce toxic masculinity, and violate female or gender non-conforming agencies. This is not a singular argument amongst KS community members and worth more attention. And I explore further below, whether these standards are reproduced as technologies of privilege.

Oftentimes, mainstream events are widely marketed to the public and accessible only to those able to pay as much as several hundred dollars for a ticket. Such mainstream sex parties in NYC and other cities in the Global North are cost prohibitive with tickets ranging from $\$ 100-\$ 150$ and from $\$ 25-\$ 100$ for single women. ${ }^{13}$ From the vantage point of my NYC field site being more integrated into the sex-positive and play party scene, there were several events that were easy to find via the Internet and social media. Because these events advertise to the general public they are considered "mainstream," in other words, not hidden from view of anyone who may be looking for such events. They are, however, exclusive in the sense that not just anyone can attend. Many of these events institute class and looks-based discriminatory policies that feel predatory, which is revealed in their application processes. These events have applications that require applicants answer intimate questions about themselves, what 
they are looking for and why, as well as submitting head and body shots - all to select attendees that are deemed a "good fit" by the evaluation of appearances. The result is often a homogenous clientele of white, physically (and some would say, intellectually) similar individuals. KS participants rebuke these normative social, sexual and beauty ideals and the organization takes steps to be representative of its community.

The publicly private (Lange, 2007) nature of KS' accessibility then predisposes the community to negotiate shifting interpretations of accessibility, privacy concerns, safety issues, and yet remain inclusive. Being located in underground spaces helps to insulate participant identities, somewhat. Therefore, one way Kinky Salon is said to differ can be found in the community's claims about inherent individual sexual agency and the right to explore sexuality. These claims are informed by ideals based on the organization's aforementioned core values. ${ }^{14}$ In contrast to mainstream sex parties Kinky Salon events are usually more moderately priced between $\$ 25-45$ per person. And the KS sex-positive ethos mandates sexual objectification be avoided and "positivity" ethics enforced. The ideological chasm that KS organizers intentionally create between their event and more mainstream sex parties and swinging lifestyle ${ }^{15}$ events, is part of what respondents state makes KS a place where vulnerable exploration can take place. In that way, participants remark about feeling freer from marginalization and shame that often result from the elitist, exclusive marketing and selection methods used by mainstream sex parties and lifestyle events. Mainstream events are viewed as lacking acceptance, intimacy, and the familiarity of community.

\section{KSL participant and volunteer, on what sets KS apart from other sex parties}

(40s, bisexual, female, white, journalist): "one of the things that I really enjoy 
about Kinky Salon is the inclusiveness. I actually have a rule that I will not go to...I'm not really interested in other sex parties to be honest but if I was going to go to them the first thing I'd do is look at their terms and conditions right?

Because I don't know what they're fucking politics are. They're always sexists. They almost always say single men can't go, no men- men activity. Fuck that shit" (KS Town Hall recording by the author, London, March 2015).

Why KS is special, or set apart from mainstream events, then becomes part of its allure - for its sex play-based connections and community that lauds sexual liberation and being welcoming to all. The KS organization thus takes pride in weaving sex into the fabric of (non) ordinary reality, claiming that it is not the focus of the party. Whereas, at these mainstream events participants claim that the focus is superficially on sex for the sake of sex. This oversexualization of sex is thought to reinscribe norms, shame, the wrongness of sex, or that sex is only sexy for the "right" kind of bodies, and that for others it is something that should be hidden or private. Adding humor and levity to sexual play enables and potentially constrains participants who (knowingly or not) engage with politics. Such political engagement is claimed to resist structures that privilege normative social conventions, particularly around white, heterosexual, bodies circumscribed by standards of beauty that often map shame onto those bodies that do not fit.

Former KSPDX co-producer, volunteer, and participant (40s, bisexual/poly, female, mixed race, social worker): "I can be shy. I can be, you know, that is where, like, I'll admit I have some insecurities because of my own body and I guess having on that costume for me makes me kind of breakthrough that mold and to -- I don't know, make me feel sexier, make me feel more confident, make 
me feel, you know, like, it's okay. I can go in there in this space because I know I look good" (Interview, January 2015).

Transformation said to occur via sexual play has been one of the most common responses I received from participants regarding why KS is so important to them. This can be seen in the vignettes and quotes above, and throughout, suggesting that either participants are actually experiencing personal transformation that they view as contributing to cultural transformation; and/or that the KS ethos is strongly ingrained in events and community rhetoric, and uncritically reproduced by participants. The Kinky Salon community's mission to influence sex culture and ignite a revitalized sexual revolution offers a deeper exploration of the making and remaking of sexual ethics for those living alternative lifestyles. Often forging into uncharted territory, for individuals' evolving personal politics and collective group politics, community members look to KS as a safe haven for more comfortably skirting the norm. Barnhill (2011) understands contemporary sexual ethics as grounded in bodily experiences of "trust, affection, care, sensitivity, enjoyment, and pleasure. ${ }^{, 16}$ Kinky Salon is then viewed as a place for experimenting with testing boundaries, broadening perceptions of what could be. For many this process is about embodying physical, emotional, intellectual, and energetic new experiences of the aforementioned qualities.

KSC co-producer, volunteer, and participant (30s, bisexual, male, white, entrepreneur): "A big philosophy of mine is to be able to actually go to those suppressed places, "tabooized" places but do it with consciousness and awareness and whatever form it is. You can have it from inside, the person, the community themselves can be conscious but this can also happen in a conscious 
environment which also helps actually bring light to those suppressed things and basically just bring more freedom. Another philosophy is also if you live out the suppressed stuff it might, in a good way, leave your body. So like doing a lot of the sex-positive stuff might not be to actually keep indulging or just doing it for entertainment [...] If it's sort of for my own sake you could say, like for my own process, I do it to open my heart and open up my body. It's difficult to use words, but open up to spirit, divine energies" (Interview, April 2015).

Energy, connection, relating to others, intimacy, and the desire to normalize the mundane reality of sexuality in day-to-day living; these are among the qualities respondents mentioned often regarding their experience at KS.

KS attendee, wider sex-positive community member (40s, fluid heterosexual, male POC, programmer and educator): "I'm looking for energetic connection now rather than just to be swinging around and whipping it back and forth and jump on every attraction [...] after the swinging experience, I realized I really wanted intimacy" (Interview, February 2015).

Quite literally, attendees are willing to be open to the flow of sexual opportunity, as "players experience a shared intimacy with others... allowing energy to flow through them" (Woodyer, 2012:321).

Wider sex-positive community member, KS adjacent (30s, bisexual, female, Cuban American, cosmetologist/professional dancer/sacred sexuality and sex work): "And that fascinates me that connection between the tangible and nontangible right. And I believe sexuality in sex incorporates more than just the physical act and more than just a physical experience. It includes your mind and 
your body and your spirit and your emotion... It's like sexuality includes

intimacy, love, connection, touching each other, listening, talking all these things right? It's not just the physical act" (Interview, February 2015).

While these sentiments expressed above may not be singular to the KS organization and community, it is the collective imaginary of participants that maintain and reproduce nuances of this statement. This further reinforces the claim that KS endeavors to normalize sex by bringing it into the conversation of day-to-day life.

KS volunteer and participant (30s, heterosexual, male, African American, selfemployed creative: "There's somebody next to us, legs over head, you know, skirt up, and we're just talking, you know. And it's kind of like, 'Oh, okay' [...] everyone was doing their thing. And that was really cool" (Interview, January 2015).

Participants often suggested that it is experiences such as these that contribute to actions that will eventually normalize sex and drive sociocultural change. As I have mentioned above, an integral part of the KS mission for participants is the emphasis on deconstructing shame sexual bodies suffer, through an emphasis on creativity, humor, and fun. Participants go on to share that sexual shame can potentially be addressed in such spaces where affect, emotion, intimacy, care, education, and community development are relied upon to try and accomplish such shifts. Doing so through facilitation of a liminal state of "becoming," these events are purposely out of the ordinary such that play defines the overall tone.

KSL co-producer, volunteer, and participant (30s, bisexual/poly, male, white, event producer): "because of the costume people could express their imagination 
or their fantasy or something else but in a way that was, you know, clearly disconnected from her day to day life, which allows people to engage in sort of a more playful way. It gives people an excuse to show off if they might normally be shy. It gives them an excuse to maybe invite other people to come interact with them. So I've seen it. It could be very powerful" (Interview, March 2015).

For KS participants, play is equally valuable as a form of resistance and can be implemented as "tactical frivolity" that includes humor and silly behavior (Woodyer, 2012:318). This feeds back into the vision for globalizing sex-positive community, claimed to be sustained by the hope of creating change. Community members do so by adopting strategies of co-creation through a politics of play and connection that are viewed as having the potential to bring about transformation. For instance:

KSC co-producer, volunteer, and participant (30s, bisexual, male, white, entrepreneur) states: "We're just trying to fucking soak them with love and often those people who are most whatever, fucked up and discriminating, they'll often be the most humble people when they leave the party. It's in that meeting, all kinds of judgment...you have to talk [and say] you cannot do this. They just need love and gentleness and basically holding space. Often what they do is they let down their whole armor and barrier, and they just feel like fuck, I've never been so welcome and they just sit in the corner and have a drink" (Interview, April 2015).

Therefore, play itself is transformed into a strategy to diffuse the tension inherent in sexual energy. Play provides icebreakers, conversation starters, laughter incitement, and the potential to diminish fear or try new things. Play gives another point of focus rather 
than someone's genitals or insecurities. It is the potential for change in consciousness and perhaps behavior that organizers and community members claim is an outcome of playful sexuality. Such play disguises subversive resistance that eventually illuminates and promotes Kinky Salon’s “sex culture revolution.”

\section{Former KSNOLA co-producer, volunteer, and participant (20s straight/poly,} male, landlord/performing artist/teacher): "I think that it's important to offer some fun ice-breaker type mechanisms and costuming is a really good one to get that shift. Usually people just use alcohol and it's something that I appreciate that Kinky Salon de-emphasizes. But on the flip side I think that it is important to encourage people to be who they really are, to express their true selves because I don't think there's as much growth if people are coming as actors than as slightly more creative and edgy versions of who they are daily" (Interview, February 2015).

But, what are the appropriate and acceptable ways to play?

\section{The marginalizing potentials of cultivating inclusive, sexual community}

Kinky Salon's mission and revolutionary vision is one of sexual liberation for all.

Therefore, accessibility and inclusivity are fundamental to the ethos reproduced by the community. To try and achieve this, organizers place an emphasis on the reduction of harm and sexual objectification, prioritization of consent as integral to sex-positive culture, including queer and body positivity. However, these claims regarding accessibility and inclusivity require much deeper analysis. While the KS positivity and inclusivity politics seem to be clear, what scholars and activists now demand for critically intervening sexual politics is an ardent commitment to an anti-racist agenda. 
Remembering that “'queers' are always and everywhere sexualized and raced... Thus race and sexuality are predominantly understood as analogous rather than mutually constituted" (Oswin, 2008:94). What does the celebration of difference really look like in these sex-positive communities' online and offline spaces? Particularly true for other mainstream events, representations are white even if embracing body positive and queer representations. The ways that race and sex intersect, particularly with regard to the reproduction of privilege contingent on difference, is something that is being collectively learned and understood by the organization and community.

KS volunteer and participant (30s, heterosexual, male, African American, selfemployed creative): "Are there people who were just scared of black men and won't be comfortable about the sex party with them there? And the answer is yes, you know, at the end of the day" (Interview, January 2015).

The quote above hones in on some of the questions raised in the Introduction about privilege and who has access to pleasure. Is it a right to engage in public forms of sexuality? And who gets to do so without consequence? The impact of the presence of blackness in sex-positive spaces is revealed in this respondent's sentiments. Sheff \& Hammers (2011) argue that people involved in kinky, perverted, or alternative sexual practices and lifestyles, particularly polyamory and BDSM, gravitate toward a reinforcement of homogeneity among class and race. So what does this actually mean for sex-positive communities like KS? Privilege in this case is revealed as the assumption of access, and that everyone feels welcome in these spaces. Suggesting that privilege is "embodied racial power" because "all actors socially regarded as 'white' ... receive systemic privileges just by virtue of wearing the white outfit whereas those regarded as 
nonwhite are denied those privileges" (Bonilla-Silva, 2017:193). In informal conversations with KS core team members, inclusion and diversity differs for each team, and teams have begun discussing what representation means, including what their communities want and need. Some teams want to provide a space for greater representation from lesbian, gay, and trans folx. Some teams want to provide a space for greater representation from people of color and differently-abled folx. In any case, an unconscious othering may be taking place in looking to include certain categories of people that are not already visibly present. The evolution of sex culture through KS has had an objective of representation aimed at cultivating the involvement of all who wish to be present. But these aims have not been evenly realized.

\section{KS attendee and wider sex-positive community member (30s, straightish, male,} black identified, IT marketing and sex-positive play party producer): “it's like many people who are in sexual communities are in like their own sort of homogeneous sexual communities [...] because that will feel safe, you know. So, like when people wring their hands about, why there are more queer people at these parties or more people of color, more gay people [...] And the answer is often, like why would queer women come to these parties when they have xyz" (Interview, March 2015).

Some participants shared that if there has not been participation of people of color, or representation of LGBTQ people at KS events, the question has to be asked why, of the right people, and then what can be done to bring about change. I asked of respondents of color: Is it pandering for sex-positive communities to seek out the appropriate channels to try and encourage greater representation? Or, is it the kind of 
activist work that goes out and talks to people not seen in the community, to see if it might interest them? Respondents seem to answer this is one of two ways, 1) talk to people and see if they're interested, and 2) why would we want to go into that space that is not created for us.

KS attendee, wider sex-positive community member (40s, fluid heterosexual, male POC, programmer and educator): "I think there are things about the community that are not inclusive and not as deliberate exclusion but it's just a non-awareness of what it takes to attract [...] So, I think that black women are the canary in the coal mine because they're the intersectionality of racism and sexism. And looking at that I'm beginning to understand that sexism and racism were not different [...] I think we need to ask black women who are exploring their sexuality in this way. What would draw them to this kind of environment? What would make them feel like they were wanted and desired and respected more than anything?" (Interview, February 2015).

KS volunteer and participant (30s, heterosexual, male, African American, selfemployed creative): "Like you can go to [mainstream] events and you can tell that they purposely don't advertise in any arena where a black person would see it [...] anything higher end, it turns very racist [...] you notice who gets screened out all the time [...] It's figuring out how to talk to the communities and people you might not be a part of [...] There are multiple layers to it. Like, you can notice something and say it's an issue. Identify a way to try to solve that issue and still never act on it [...] the only way you get these people is going to their 
channels. And it's not fetishizing [...] go through their information system chains" (Interview, January 2015).

A common response among those who felt "othered," was that underrepresentation could be remediated by a few things: access to information needs to be presented to familiar networks; making it a point to speak directly to the groups of people you wish to see attend; understanding why people do or do not attend, and why it is seen as important to have them attend; and getting feedback from people you would like to see at events. These being some of the ways to establish a dialogue and potentially open up the ideological space that may have been regarded as "not for me" by some. It was recounted on a number of occasions that from an outsider's perspective KS events appear predominantly hetero- and bi- friendly, are still very white, and that there are other spaces that cater more intentionally to LGBTQ or POC folx so why bother with KS. And the costuming is truly not for everyone.

Another challenge to inclusivity is the potential for class-based exclusions, partly due to ideological grounding in tech/creative structures. For instance, access to the community via the Internet, the cost of admission, a willingness to engage in costuming, transportation, and refreshments, can be burdensome for participants. The PAL system is one such structure that sometimes makes it difficult to get started, especially for people new to the community. As I mention previously, this system was created to develop accountability and encourage deeper participation among community members. The organization considers this process one of intuitive and community-based feedback, which is stated to have been the primary way Kinky Salon has evolved over the years including its core set of ethics foregrounding play, fun, and inclusion. But the need to 
create connections in order to participate, for some new to the scene, can all but eliminate their participation. If a person knows no one in the community, or has no like-minded friends, it can be a challenge to attend. This has been acknowledged as an obstacle by many, including the "Mothership." To remedy this, local KS chapters are encouraged to reinforce their community-making by hosting and sharing other events outside play parties. It is said this encourages people to get to know potential PALs and make other connections in the community. However, my experiences and observations suggest that this doesn't work for some people and leads to feeling marginalized. Some of the questions I asked myself after feelings of initial awkwardness reflect these internal tensions: Is sexual liberation just for those outgoing, fun-loving, gregarious, adventuresome people who make connections easily and aren't overly concerned with whether someone will 'pick me' for some fun? What if your PAL is having more fun than you?

Furthermore, I met no one at these events specialized to manage the tough emotions and traumas that may eventually surface for people in sexual situations. After all, one of the reasons heralded about the positive affects of sexual community is the ability to work through or address sexual trauma, or the understanding that it may come up. However, walkaround hosts are not first responders, nor do they have any specific mediation or trauma training. When asked to check in on guests as part of the volunteer role there are no hard specifics regarding to how to deal with tough emotions that could be triggered or traumas that could surface. I have seen one woman have an emotional breakdown at an event, where drugs and alcohol were contributing factors. Volunteers sat with and talked to this woman for some time. She was having trust issues come up with 
her PAL/partner. Volunteers were able to calm her down by taking her aside, getting her water and talking to her. Other guests were visibly affected by the scene, demonstrating discomfort about the incident by keeping a concerned, watchful distance. This moment highlighted how sex can be emotionally or physically traumatic for some. And, I became curious about whether something like this could be dealt with in community by having the appropriate tools, rather than quarantining the person to be attended to separately.

When it comes to such contestations of intimate space, one way the complexities of play are brought to the surface is through consent violations that may occur at events and need resolution. As I unpacked the alignment of the KS ethos with practices, I found that the ways allegations against the community are dealt with is an example of the tensions that exist therein. Among the wider sex-positive play party community, when consent or safety violations occur, members may use the Internet and social media as tools to speak out about concerns. Though taking more direct action, a San Francisco non-profit called "Consent Matters" recently attempted to establish a local database and communication network for all San Francisco play parties. Unfortunately, the group was unable to establish consensus for an approach considered to be both ethical and effective. A central issue is how to keep the database of names private and secure in the event of a lawsuit.

In the New York sex-positive play party scene, and within the KS Global Community (KSGC), there is a push for mobilization to have policies in place that deal with and effectively prevent violations. For instance, there was an incident with a KS volunteer also involved with other NYC "exclusive" play party scenes. This volunteer gave the KSNYC team a list from another event stating the team had permission to use it 
to email invitations out for events. However, due to an internal conflict between this volunteer and the person from whom the list was received, KS did not have permission to email these people. This was discovered after an event email was sent. The KSNYC team received an email from the group where the list was generated to speak about the violation of consent that had been unknowingly breached. Exemplified here is an excerpt from the internal email:

"I need to know how my email is getting on other events' lists. We act as though we have a safe and exclusive community that recognizes the need for privacy, but perhaps my comfort levels within this community are unfounded and my expectations too high. This is the second time my email has been given to another list. It is not ok. I am essentially receiving spam. My name and personal information are being given to people and events I do not know. I would rather not be invited to any events than have to worry that my personal life may be exposed due to carelessness on someone else's behalf."

As a result of this, tighter policies were developed around the use of people's personal information, with only direct opt-in to email lists being the agreed upon method.

Another incident with the KSNYC team involved a member of the wider New York sex party community, who was recently accused of partner rape at another event. This person bought tickets to an upcoming KS event. The KSNYC team was petitioned by a community member who knew both people involved, urging the team not to allow the accused rapist to attend as a matter of maintaining safety. The team got the "Mothership" involved to discuss ethical and legal issues. Because this incident did not take place at a Kinky Salon, it was decided to refund the person's tickets and ask them to 
step away from the event until the matter was clearly resolved between both parties. The outcome here was to maintain the safety of the space according to those who spoke up about being triggered, but without getting more deeply involved in the matter, or the welfare of the person who brought the allegations. By contrast, for Kinky Salon community members, the terms of reporting violations of consent and safety allow for case-by-case review of incidents, holding all parties accountable and requiring their input for resolutions. Information sharing in these cases is one way the community educates its members and tries to model safety through communication strategies. However, this also clearly highlights the fragile nature of participation in these communities, and that the fear and consequences of being exposed are real. I go on to explore further the consequences of sex culture activism in the following chapter.

\section{Conclusion}

The two excerpts that began this chapter highlight what is at stake for the KS organization and community. This organization and community would not be perceived by participants as revolutionary and transformative were it not for the safe, creative, playful connections made that encourage sexual exploration. The data thus demonstrates how affect, intimacy and embodiment are key to encouraging such openness to engage with (sexual) play at KS events (Bain \& Nash, 2006). Play is what opens the door for people to experience new ways of being (Hayes-Conroy, 2010b), particularly as part of a sexual community (Weeks, 1996). Therefore, for the KS community, sexual play is the primary way in which transformation is envisioned. As I noted in the Introduction, Woodyer (2012) considers play as a characteristic that is present in behavior. Also reiterating that play is relevant and enacted in the everyday - in the personal and the 
political - often beyond the reach of representation or rationality, it nevertheless has the potential to instigate reflection that may prompt transformation (2012:316-22). In this chapter I have demonstrated how this happens as a politics of play and the creation of safe spaces to connect. The commitment to play and the subtext revealed through it is often informed by contextual sociopolitical realities of the place and space in which play is enacted (Rahier, 2013). Although, "subversion often takes the form of rational critique of the established order" (Turner, 1979: 494), it can also take the form of irrational, humorous, absurd, or irreverent commentary and critique. Volunteers and community members produce spaces where people can experiment with their sexuality, unconventional relationship styles, body positivity, kink, and communal sex, or be supported while processing the physical and emotional aspects of pushing one's own boundaries. These explorations are fraught with the tension of trying to be accessible and inclusive to everyone interested, while also encouraging the interest of those that believe the spaces of sex-positive community are out of reach. This chapter also demonstrates however, that the hope for change is enacted through the potential for sexual liberation encouraged through sexual play. Although the reality of such accessibility is obscured when change is unevenly constructed, and erases who might be marginalized or remain unheard. Therefore, making a personal investment in a politicized, sexualized space is also often entangled with, and complicated by, the prioritization of such idealized change.

In the following chapter I will explore the care practices that I argue are embodied as sex culture activism, enacted through "radical acts of being," community-making and education. 
${ }^{1}$ For increased visibility around once-taboo sexual practices, see: Attwood, 2014; Califia, 2000; Comella, 2014; Herman, 2007; Weiss, 2006, 2011; Williams, 2004; Wilkinson, 2011.

${ }^{2}$ A concept used by the KS founder, participants, and sometimes heard generally in play party and Burning Man scenes, to distinguish from superficial hedonism. The connotation is that deep hedonism has value that is manifest differently, in alignment with care ethics. This is something one comes to understand only through direct contact with the community in terms of their specialized language. There may be differing descriptions of this term, but this is an unspoken collectively agreed upon (tangential) conceptual framework.

${ }^{3}$ I am using the adjective softcore here as a metaphor to suggest that the sexuality activism engaged with by the KS community is more oriented toward normalizing ethical hedonism, and may be seen as superfluous to previous movements of the 1960s, 1970s, and 1980s, with less collective mobilization and alliances, and no direct action against institutional structures to agitate for change. Nevertheless suggesting that transformation which begins with the self, also creates a ripple effect of change on a broader scale. Softcore (Dictionary.com): Adjective of, relating to, or containing sexually arousing depictions that are not fully explicit: soft-core pornography. (Urbandictionary.com): Pornographic material that does not show penetration, genitalia, or actual sexual activity, opposite of hardcore.

${ }^{4}$ Public sex, radical sex, sex publics: Albert, 2011; Berlant \& Warner, 1998; Califia, 2000; Castiglia, 2000; Dangerous Bedfellows, ed., 1996; McGlotten, 2013.

${ }^{5}$ Boylesque is a contemporary addition to burlesque entertainment, part of a neoburlesque movement, dedicated to gender non-conforming, or male performers.

${ }^{6}$ A term Cooper (2009:105) uses for "organized social practices that neither replicate nor straightforwardly reverse hegemonic relations".

${ }^{7}$ Although during my time collecting data, I never saw this system break down. I only witnessed (and experienced) the main problem of being unable to find a PAL.

8 "DO: Be creative about how you dress; Contribute when and where you can; State your boundaries; Play safely and consensually; Have sensible safe sex practices; Respect our space and each other; Clean up after yourself.

DON'T: Linger unaccompanied in play spaces; Cruise aggressively (even if they are really cute); Get too intoxicated; Take photographs; Use your cellphone; Gossip about what goes on here; Leave without your PAL."

${ }^{9}$ The idea of a safe container is derived from psychotherapy and self-help language. It refers to "holding space" for someone, or creating a "container," to help people process 
experiences of grief, trauma, depression, or other transitional emotional states. There are a number of techniques that taken together help create such space: practicing loving kindness, using deep listening, having unconditional positive regard, sit with what is, allow, breathe, get grounded, be present, do not usurp their pain, practice non-judgment, and do not try to fix it (https://www.goodtherapy.org/blog/11-things-that-will-help-youhold-space-for-someone-0523175).

${ }^{10}$ Cosplayer: "a person who dresses up like a character from a manga or anime series, a movie, a videogame, etc" https:/www.urbandictionary.com/define.php?term=cosplayer.

${ }^{11} \mathrm{https}: / / \mathrm{www}$. teenvogue.com/story/consent-how-to

12 Typically dungeon equipment is used in the practice of BDSM. However, KS calls this space the FUNgeon, to keep the BDSM for people that enjoy it, but to also keep it light and integrated into the fabric of the event.

${ }^{13}$ An example of some of these costly mainstream events: Behind Closed Doors (NY)/ School of Sex (NY); Top Floor (NY); One Leg Up (NY) - considered to be one of NYs oldest mainstream sex party; Killing Kittens (UK, US, EU, Canada, Hong Kong, Australia, Germany, Switzerland); Castle Events (Europe); Torture Garden (UK).

${ }^{14}$ Playful, safe(r), inclusive, creative, community spirited, socially conscious, sexually progressive (https://www.kinkysalon.com/the-7-core-values-of-kinky-salon/).

${ }^{15}$ In sex culture communities the term "lifestyle" is often used as a short hand term to imply swinger, or swinger events. Swing lifestyle is characterized by the colloquial "wife swapping" practices that became popularized in the 1960s and 1970s.

${ }^{16}$ See also Lawson, 2007, 2009, 2011. 


\section{CONSTRUCTING CARE IN SEX CULTURE: Reimagining revolutionary change one educated orgasm at a time?}

"Sex Culture isn't just about sex: It's about art, community, spirituality, relationships, gender, family, self-expression [...] Sex Culture supports all choices and orientations between consenting adults, and sees them as part of a complex, cross cultural, sensual and aesthetic exploration." (Excerpt from Polly: Sex Culture Revolutionary).

"The productive capacity of care is its power, and power is conceptualized through the lens of attentive action, with a force that is a "necessary attribute of social action'"' (Cooper, 2009:109).

\section{Introduction}

In the previous chapter, I highlighted the ways in which sexual play is constructed as political. I demonstrated that play is a vehicle through which participants may begin to realize the so-called transformative power of group sex. The type of vulnerability encouraged through sharing and becoming an open participant, is made more possible (palatable?) and enhanced through play. However, the mission of sexual liberation is complicated by exclusions that may reproduce class and race based sociocultural and structural inequities. I concluded the previous chapter by pointing to ways the inclusivity ideals of sex-positive community are challenged. These challenges potentially deeply undermine an ethos of being sexually liberatory for all.

In this chapter I assert that the discourse within feminist and geographies of sexualities have yet to fully engage with sexual intimacies as a form of care and the basis for social change. Historically, the fight for rights through revolution have often been prompted by extreme need. For instance, sexuality became overtly, and publicly political with the actions of the Gay Liberation Front and the impact made by public activism groups like Act Up (Evans, Gosse \& Moser, 2003:194) in response to violence and hate 
crimes. So to, it was in the midst of the AIDS crisis, gay and lesbian activists, developed knowledges of safe sex and its practices (Evans, Gosse \& Moser, 2003:206). These knowledges are among what are considered fundamental safe sex practices in sex culture today. This case differs from the care tactics practiced by sexual dissidents during the 1980s and 1990s in response to the AIDS crisis, however. While safety and risk in sex have been theorized in terms of preventing HIV and STIs, following Barker, Gill \& Harvey (2018), this case study answers the call to explore how "forms of emotional risk involved in sexual activity" are also of concern, yet seldom analyzed (2018:1340). Accordingly, I will illustrate how affect, emotion, and intimacy are embodied through care practices that are linked to the cultural and sexual politics informing social change objectives within sex-positive community.

\section{Intellectual setting and methods}

Social organizing and activism has been a key feature for mobilizing ideas of sexual revolution since the late 1960s. However, sexual revolution is a concept that is often placed in a past tense context, which this case seeks to demonstrate otherwise. Since the early 2000s, Lenius (2011) argues that LGBTQ communities have been influencing sex culture, particularly with reference to BDSM and alternative sexual practices (swinging, group sex, fetish/role play, etc.). And, Nicholls (2007) affirms that new social movements - such as "sexuality, environmental, minority, and regional movements" have "crystallized around issues of regaining autonomy and control over identities and cultures" (2007:610). It is claimed that the ways dissidents develop social networks can tells us something about the nature of the oppressions faced and strategies used to mitigate such pressures (Aitchison, 1999). Such that there is a need for "a redefinition of 
political involvement that emphasizes diversity and subjectivity" suggesting that lifestyles and cultures today are a "rich source of movement mobilization" (Della Porta \& Tarrow, 2005:13).

The Kinky Salon ethos is developed around the claims that, "sexual revolution brings a revised idea of sexuality. That it's healthy, it helps people bond, it feels good, and is actually good for you. Sexually progressive culture is a place where these new ideas about sexuality are being embraced" (Founder Interview, February 2014). Supporting this idea, Giddens (1992) argues that "sex now speaks the language of 'revolution': it is de-centered, freed of reproductive needs, and thus transformed" (Ross, 2005:342). This view removes the solely procreative utility from sex and restructures it as a social mechanism. Scholars suggest this new turn, or neosexual revolution, is therefore unique due to contributions from critical theory, pornography, and the Internet (Garlick, 2011); and also an understanding that the AIDS crisis of the 1980s into the 1990s created an atmosphere of fear around sex (Meunier, 2013).

A vision of a "sex culture revolution" then, is a concept rebranding the past and being carried forward. The use of online domains as organizing and community building tools extend the reach of sex culture and community beyond the material space of each event. And, offline, real-time events are intertwined with and promoted through online forums and social networking communities. I prioritize claims made by scholars such as Bardzell \& Bardzell (2010), Garlick (2011), Karl (2007), and McGlotten (2013), who argue that the Internet and social media are spaces of performance, sexuality, a tool for sexual revolution, and for the management of everyday life. And reinforce the importance of play as a predecessor to building an ethics of care within sexual community. This 
supports the move away from physiological explanations for sex, to psychosocial interpretations for the benefits of sexual activity. For the KS community, "sex culture revolution" prioritizes what organizers call "heart-centered relations," emphasizing the need for "conscious connection" through community-making efforts. Such efforts are conceptualized among various care practices. Such practices suggest that, according to Victoria Lawson (2007:3): "Care ethics begins with a social ontology of connection: foregrounding social relationships of mutuality and trust (rather than dependence)." However, a critical analysis of care ethics must also be concerned "with mutuality and well-being... and also demands attention to emotions and affective relations...because of the complex ways in which power is embedded within them" (Lawson, 2007:3). This is an important intervention to interrogate how care and power are bound up with sex. Recalling from the Introduction that "care is equally the site of the reproduction of existing power relations and oppressions" (Tauqir et al., 2011:18). To more deeply explore these themes in this chapter I answer the following research questions:

Theoretical question: What are the implications for care practices to create sociocultural change around sexuality today?

Empirical question: How is an ethics of care enacted at a play party? Within sexual community? How do care practices contribute to sex culture activism for community members?

I first articulate why it is important to pay attention to revised understandings of neo sexual revolution. Then, with the use of interview and observational data, I interrogate how care is constructed as part of a social change agenda; examining how care is deployed in a sex-positive community. My time as a producer and participant 
enables me to examine how creating spaces for sexual dissidents is connected to providing support for resistance to institutional and structural norms that stigmatize socalled "perverse" sexual expression. Conversations and reflections reveal how care is enacted to deconstruct dominant modes of thinking about deviant sex. Though I question the challenges of constructing a brand of activism reliant on social networking used to connect people and ideas, in what I understand as a softcore sex culture activism. I do so by demonstrating attempts to embody an idealized sex-positive ethos makes available an easy, practical activism, which many participants claim is useful because it is realized through community. Finally, I reveal how failures of care (Cooper, 2009) in a sexpositive community become embedded in a colorblind liberalism that obscures structures of privilege (Bonilla-Silva, 2017).

\section{Reimagining sexual revolution as softcore activism through a politics of care}

If the sexual revolutions of the past have done so much work to attain rights for sexual dissidents, then how can we conceive of sex culture activism today? What this activism through Kinky Salon looks like for some, revealed here, demonstrates how care is articulated as positivity discourse that is credited for instigating change. The overwhelmingly common tone of sex-positive community members is romantic, idealized, and emblematic of the hope expressed early on. Such hope is that which many participants of KS have reported feeling by helping to build the community:

Former KSNOLA co-producer, volunteer, and participant (20s straight/poly, male, landlord/performing artist/teacher): "For me Kinky Salon is about activism. It's a channel through which I can send energy for positive change [...] what will be done away with will be liberating, what will be changed will be 
revolutionary. [...] We are indoctrinated so early that even the most open minded of us may not have considered the alternatives. Which alternatives? Through community we can make this happen. Through community we can outsmart and undo relationship expectations levied against us from cradle to coffin. We have the power, the inner beauty, and the human right to engineer the agreements of our own relationships" (Interview, February 2015).

Cooper (2009) argues that the power of alliance such as this quote demonstrates, coalesces around attentive action. Also, reaffirming the quote at the beginning of the chapter, which links attentive action to care and intimacy through play. The sex culture revolution (SCR) agenda, in $\mathrm{KS}$ terms, is not organized as a typical social movement, but organized in such a way that "weak ties"1 (Nicholls, 2007) define individuals' investment in general. Although not a typical activist organization, the underground, loose organizing of those involved create visibility around diverse matters concerning sexuality. ${ }^{2}$ Many participants I spoke with strongly favor the KS sex culture revolution ideology. Often however, community members become involved in a type of softcore social activism through part-time participation in pushing sex-positive agendas via social media outreach and information sharing.

As mentioned previously, the KS community of participants and volunteers are often engaged in lifestyles that take on aspects of relationship anarchy or ethical nonmonogamy. By virtue of practicing non-normative relationships and sexuality, individuals report feeling marginalized and stigmatized, embodying sexual dissidence. Respondents suggest that the very embodiment of being sexual is transgressive. I interpret this as informing a matrix of resistance that structures the ways many 
participants move through the world. Always fighting for something, namely, the right to $b e$, echoes a respondent's earlier sentiments about the political nature of the right to be left alone. For the KS community these understandings are realized as emotional labor contributing to a productive form of care. As such, sex-positive ideals are proliferated through a cross pollination among and beyond KS community members at socials, events, online and offline. Members of the sex-positive community for whom alternative lifestyles and practices inform their daily lives, may be situated as activist-oriented by default in finding themselves in the KS world. The community is thus comprised of individuals, some of whom self-identify as participation and activism-oriented, politically aware, and concerned with care ethics at multiple scales.

\section{KS participant and volunteer, former KSL co-producer (40s, queer/poly, femme,} Caucasian, pornographer/writer/activist): "For me, it is --it's part of my activism really in a lot of ways, you know, shared resources is very important to me. Again, mutual care is very important to me, so all of us having other people that we can talk to and be intimate with and like underneath that is really important" (Interview, February 2015).

At the other end of that spectrum is the act of trying to actually change policy, which is not necessarily happening directly in relation to the KS mission. Conversations with sexpositive community members suggest that sexual politics are important to them, even if some are not involved with politics directly. However, respondents' who do engage with sexual politics discuss their participation in relation to careers and passion projects. They ranged from being a sex worker advocate for an online forum providing access to work; a sex therapist and sexologist; plutonic touch and cuddle business owner; porn writer and 
editor; queer, feminist porn creator; sex educators; professors; and a mental health counselor focusing on intimacy and trauma; to name a few examples. For each, a commitment to change-making is consciously or unconsciously made that requires transgressing norms regarding their choice to work with sexuality and intimacy. This offers a perspective of how the claims of changing sociosexual norms operates at the micro level of community. Strengthening the claim that "the bubble of activism for KS is about where the act of doing something subversive is an act of activism." For the community, “it's more about art, it's more about inspiring people, and it's more about having the activism be in the radical acts of being" (Founder Interview, June 2018). For many participants, and even KS organizers, politics are still a somewhat touchy concept to have dominate a fun, sexy party. Interlocutors report wanting the politics to be anti-political, artistic, action oriented, and more about having real conversations with people about their beliefs.

KSC co-producer, volunteer, and participant (30s, bisexual, male, white, entrepreneur): "Basically I think the best political agenda is I sit and talk with a guy who's head of some ministry. He went to our party and he's transformed [...] I talked with him about what is sex positivity, what is gender, what's going on. [...] It's action-based. It's not about talking, making political statements. It's about our appearance in the world. It's about people who have gone through it. It's about the lives we changed. That's a political agenda, that's our political action. We're not trying to tell anybody what's right and wrong. If they enter the door, we'll change their lives and we'll spit them back into society. That's it" (Interview, April 2015). 
Many participants declare themselves to be intent on pushing back on what it means to exist in a sex-negative world. It comes down to the opportunity to "establish an 'affinity'... defined as a 'feeling of connection' between people who [experience] an openness to interacting with another person" (Lange, 2007:363). For instance, one KS London co-producer states: "there's a strand of our community that want to engage because they like their liberation or political aspect but they don't really want the sex aspect" (Interview, April 2015).

Relationships and connections that develop into alternate kinships are key to such affinities for sex-positive communities. It has been argued that LGBTQ relationships "should be viewed as an alternate form of kinship in contemporary life" (Brown, 1997:121). Alternate forms of kinship can therefore be established as a type of care work, relied upon for support. Non-normative relationships and sexual practices marginalize members to differing, intersectional degrees. A sense of community is thus reinforced and strengthened because "community is in fact likely to come from those groups who find the premises of their collective existence threatened, and who construct out of this a community identity which provides a strong sense of resistance and empowerment" (Weeks, 1996:72). According to Cooper $(2009: 106,107)$ an "intentional (utopian) community" such as KS, facilitates use of "everyday utopian space" as a space preoccupied with being motivated to democratic and egalitarian methods of operation. These ideals are akin to the KS ethos that articulates the mission of making the world a better place "one pervert at a time." ${ }^{3}$ This ethos in action is accomplished through an emphasis on accessible and inclusive consensual (sexual) play, said to be a catalyst for deeper connection through the care of intimacy found in community-making. 
As an example, ways care is enacted by the gay community in Vancouver include practices like "Advocacy, counseling, education, financial aid, food, home support, housing, legal aid, (care) support, testing” (Brown, 1997:49). For the KS community, care shows up in equally myriad ways: Playing (board) games together at make out parties, and a tea area at events for non-alcohol related socializing. Or, a meet up group called friends of Kinky Salon in the Bay Area. ${ }^{4}$ The organization has established art grants for local artists to share their work with the community, and highlights crowd funding for creative endeavors like album production of a local comedic singer who is also a KS cabaret performer. Mobilizing the community to vote and using social media platforms to speak out about sex work, abuse and violence against sex workers is also common. All the while trying to stay on top of the constantly changing privacy rules on social media that effect the naming of people, yet remaining accessible is a complicated task. Frequently, social media "signal boosts" are used to send out calls to ask for help, to help community members crowd fund for medical and hospital bills, to take care of each other when people are sick, or for sharing wellness and healthcare information and general emotional support and understanding. This demonstrates part of the framework for this community's organizational priorities and formulaic structure. These structures and practices serve to reinforce engagement by/with the community. For participants, KS acts as a facilitator of opportunity, with aims to promote change and create the space for people to regain control over their sexual identities.

In these ways, the Kinky Salon event and community provides an opportunity to understand sex-positive culture in relation to a particular sociocultural mission. For many community members, the emotional qualities of care that are mobilized by togetherness 
has the effect of coalition building and adds a utopian quality to various levels of participation. In order to more fully understand the consequent investment in sex-positive community and culture, and for some, direct action, I have explored how community members make their own distinctions about what it is and the relevance it has in their lives. For the community, paying attention to and being informed about how to operate in sex-positive ways is one presumption of enacting care.

Wider sex-positive community member, KS adjacent (30s, heterosexual, female, white, photographer): "The more communities we have that are sex-positive means more education, more consent and people living their lives focusing on positivity versus negativity. That benefits everybody" (Interview, March 2015).

Idealized within this framework, a community of individuals with similar values and belief systems take the shared risk of engaging with vulnerability through intimacy. This suggests that action is mobilized around the commitment to continue having sexy fun, which in itself is valuable (Cooper, 2007). For instance, participants are drawn to sexpositive communities via a belief in the potential for pleasure the connections hold:

Wider sex-positive community member, KS adjacent (47, white Latino, female two-spirit, energy worker): "Physical, mental, emotional, and spiritual health is all based on having a healthy libido, which one is, you know. And, so that's like primary and fundamental to everything. Any sort of pleasure in life is derived from having an extensive experience of cracking libido in the body. It is literally energy"(Interview, January 2015).

However, it has been argued that pleasure needs to be more carefully unpacked to get at the "multiple potential pleasures possible from sexual and erotic experience, or of 
the complex interweaving of pleasure and other experiences in sex" (Barker, Gill \& Harvey, 2018:1341). The idealized seeking of pleasure or the perceived right to pleasure may also reproduce an uncritical view of what pleasure is and the privileges associated with it, deserving a closer look at how this operates. A critical analysis of pleasure in sexpositive community demonstrates the potential for pleasure to be unevenly realized through "attribution error" (Doane \& Bonilla-Silva, 2003). This perspective suggests that if one makes the effort, it is possible to achieve through hard work or the intentional seeking to change one's circumstances - expecting to receive the benefit of these spaces simply for being in them. This view forecloses the realities of the historical inaccessibility of pleasure for some, recalling the discussion of being Black at Burning Man; suggesting that essentializing pleasure obscures the obstacles folx may have to surpass to initiate radical acts of being. This kind of mentality is interrupted however, by the emphasis the community places upon calling out/in/on each other, embracing emotion, and the experience of needing each other. This suggests that care and pleasure are at best, co-constituted, (with the pleasure being the giving or receiving of support), for instance:

\section{KSL participant and volunteer on what sets $K S$ apart from other sex parties} (40s, bisexual, female, white, journalist): "It's the connections. These connections are lovely. They're generous, they're kind, they're heartwarming, that's what actually builds a community, that's what people are connected to. It's not just about going to an event, paying money, receiving some kind of, I don't know, reward for the money. It's about an interaction and the connection within the community" (recording by the author, KS Town Hall London, March 2015) 
The care practices that are said to facilitate sex culture change are enacted through playful shared intimacy, education, focused within community. Education and community-making is accomplished by adopting strategies of collaborative creation of space to gather and share ideas. This became evident after receiving numerous emails about joining in for game nights or pot-lucks. Sex culture activism, in these terms is then understood as being personal, requiring community contribution, being accountability oriented, which contributes to revolutionary cultural change. Yet, this emphasis on ethical hedonism may also undermine access to care for sex-positive play party-goers that feel displaced and unwelcome in such communities. I go on to explore how care is central to such activisms further below, followed by the impacts of unevenly realizing care.

\section{Enacting care ethics through radical acts of being, community-making and education}

As I have shown earlier, the care practices that influence sex culture change for this community are firstly embodied through a politics of play. I detailed in chapter three, that the creation of a safe space, or what the community calls a "safe container" is a crucial element of the framework for play and change to ensue for KS community members. Importantly, for community members such play is supported by care practices that prioritize sex-positivity, connectivity, information sharing, and overall community investment. Sexual adventuring as a reason enough in itself to matter, can be regarded as how "care's concerns often do not come from emotional connections between partners but reflect, other, largely external, forms of mattering" (Cooper, 2009:112). Playful sex and sexual adventuring is thus linked to care through "forms of mattering" that are revealed in practices of intimacy for community members. I myself have felt like I 
mattered as a member of the community being cared for when I was ill and feeling isolated.

I have found that it is the sexually charged, playful spaces created that facilitate vulnerability among participants. Within this vulnerability is a willingness to listen, open up, observe, and communicate clearly - if for no other reason than to maintain safety (Weiss, 2011). This recalls Woodyer, Cooper, and Edensor and Bowdler's corroborative claims about the power of opening up to affective immersion in the moment, playful vulnerability, and the ability to instigate change due to surrendering to the moment. Such opportunities to practice care, Lawson (2007) suggests, include values of empathy and attentiveness that are also spatialized as “caringscapes," (Lawson, 2007, 2009). Kinky Salon organizers can be said to create caringscapes for events, and can likewise be signaled as a community that is itself a caringscape. Such caringscapes "treat different scales as mutually constituting and connect multiple sites of care. Central to this approach is the negotiation of different discourses, demands and actors in shaping situated practices of care" (Lawson, 2009:567). Translated into sex-positive terms then, care ethics are enacted by placing an emphasis on safety and consent, positivity ideologies (sex-, body-, LGBTQ), endeavoring to be accessible to the community, and holding space as a sexual community that welcomes a full representation of voices. Investigating further reveals what care looks like and what it actually does when influenced by the politics of sex-positive community.

For instance, community members are used to looking out for each other's safety and well-being (i.e the PAL system). Participants at events claim to feel at home, safe enough to literally strip down to their vulnerable self. In this safe container there is a 
volunteer walkaround host whose job it is to invite people into the space and make them feel welcome, check in on guests as the evening goes on, mediate any problems or incidents, and generally help create an atmosphere of conviviality. The spaces of caring at events can be experienced in different areas geared toward non-sexual bonding and intimacy. There is a no-play zone, ample space for dancing, socializing areas where people can relax and have non-alcoholic beverages. As well, spaces outside events that are similarly constructed. Thus, care is enacted through the idea of connection not centrally focused on sex. Instead, prioritizing relationship building around shared intimacies found at: non-sexual socials and gatherings, and even calls for the community to help with the upkeep and maintenance of the venue grounds, gardens, rooms, and make general improvements. Other resources for support are found on the KSSF website's community connection tab providing links for support/therapy: "Bay Area Open Minds," referring practitioners in the Bay Area specialized as "psychotherapists and psychotherapy students who affirm that sexual and gender diversity are natural expressions of the human experience." ${ }^{5}$ Therefore, in order to facilitate opportunities for playful, transformative sex, this sex-positive community creates multiple avenues for vulnerability to be realized through practices of care. Thus, community integration activities are important for people to connect with others with whom they may not come into contact outside the space of the event. Workshops like "Effy Blue's Play Party Etiquette workshop;" "Hands On Rope Work. A very practical rope demonstration and practice class;" and "Orgasmic Manifestation, presented by The Sex Sensei! A workshop centered on experiences and definitions of orgasm, working with people's sexual centers, 
creating affirmations, intense breath work (orgasmic breathing) and magical tools to continue manifesting the energy!;” are a few such opportunities.

For participants, care is embodied in the de-emphasis of sex and the use of its energy to build sociality that helps normalize the expression of sexuality and reprogram what it means to be a sexual being:

KS attendee and wider sex-positive community member (30s, straightish, male, black identified, IT marketing and sex-positive play party producer): "for people to treat these spaces as high possibility low expectation spaces isn't just about, you know, creating a safe culture although that's certainly true. Like it's also a call to, reorient the way that many of us have been trained to think about sex as, like, this super high stakes thing, right?" (Interview, March 2015).

Former KSNOLA co-producer, volunteer, and participant (20s straight/poly, male, landlord/performing artist/teacher): “Kinky Salon community puts its influence less on the actual physical interactions, less on the actual sex and more on the relationships, more on the ways people are interacting with each other, more on what might come before the sex, how people connect leading up to having sex than the actual sex they're having" (Interview, February 2015). KS attendee, wider sex-positive community member (40s, fluid heterosexual, male POC, programmer and educator): "The dancing, the gentle mingling. I think the feeling of sexual energy that's in the air and that there's no -- the lack of oppression around it” (Interview, February 2015).

Organizers points out that being around sexual energy and learning how to engage with it in different ways, can help people become more socially conscious such that it impacts 
even mundane aspects of day-to-day living. These distinctions made between such mutually constituted scales of care, are created through the "negotiation of different discourses and demands" of the community, as Lawson (2009) earlier suggests. Demonstrating also the "vernacular knowledge" (Escoffier, 1998) implied in such learnings is collapsed into affect, emotion, and intimacy constructed as "energy" flows that are also connected to care practices.

Going further, Lawson (2009) claims that such spaces work to deconstruct the binary of public and private. And I argue that caringscapes developed around varying definitions of intimacy further challenge this public private divide, particularly when enacted in a sphere that is outside the home, in a more "privately public" (Lange, 2007) space. For instance, Kinky Salon has been created and grown with the input of the community. Reciprocation, acceptance, and connection are reflected as important qualities of the community for participants. Hearkening back to the BM principles introduced earlier, which many community members are familiar with. With all the intentionally inclusive language and ideologies that the KS organization models, interviewees reflect that it amounts to demonstrating new ways of being, particularly in relations to others, that deconstructs shame around bodies and sexuality:

KS attendee and wider sex-positive community member (39, Caucasian, male, straight vicarious, writer: "Sex positivity, I would say that term to me is trying to get rid of the shame. It's trying to de-shame sex in general. Sex positivity means people who are happy with it, they are positive about it, they think it's a good thing for people to do [...] So I think, by removing the racism about it, and removing the fear about it, and removing the shame about it by having sex- 
positive community is to do away with a lot of [what] we are dealing at a society level" (Interview, February 2015).

In this way, care practices also serve as the basis for some community members to combat stigma and/or shame. In a study about HIV and AIDS related stigma, Parker and Aggleton (2003) offer an empathetic view of stigma that reads the experience in the context of social structures, and recognizes the potentialities of mitigating such individualized pressures: "Stigma and stigmatization function, quite literally, at the point of intersection between culture, power and difference - and it is only by exploring the relationships between these different categories that it becomes possible to understand stigma and stigmatization [...] as central to the constitution of the social order" (Parker \& Aggleton, 2003:17). But even with the education, safety, and consent components in place, the stigma attached to such lifestyle choices remains a heavy burden for many of the people I spoke with. Therefore, the mediation of such stigmas also informs the carebased KS brand of sex culture activism.

Throughout the data collection period, respondents have articulated the real world consequences for participating in playful sexuality within group settings. I have known a professor at a liberal university that could not be open with their sexual practices fearing loss of tenure and ostracization. The fear of job loss was not an isolated remark. Child custody battles, divorces, and domestic violence have also been reported as part of what complicates the daily realities of living with alternormative sexual desires. In fact, many respondents take the idea of education seriously as a way to manage and hopefully diminish stigma over time. 
KS attendee (30s, heterosexual/poly, male, Caucasian, writer): "I think that sex has a stigma against it within most of society and my goals in life are pretty much to live my life by setting the example that I wish to see in society" (Interview, February 2015).

KS attendee (30s, queer/poly, female, Caucasian, business operations manager): "one thing that I've seen as a result of especially Kinky Salon is a groundwork for learning and communication beyond the sex education in a classroom. It extends in our community [...] that experience... pervades so many other types of experience and communication. I think it can really only help. I think it's really important" (Interview, February 2014).

These respondents demonstrate that being able to talk about alternormative sexual practices, first in a safe community, enriches daily life. And some participants are then inspired to carry the message further afield. In these respects, participants acknowledge the part they're playing in the KS sex culture revolution mission. Therefore, if for many of these respondents, shame is indeed bound up with experiences of sex and sexuality, it affirms Mercer's claim that shame is frequently used to frame sex/uality (Mercer, 2018:1304). It is then a powerful driving force that can be used in service of subjugation and control, being doubly impacted by both the feeling of it and the subjectification of it (Mercer, 2018).

Education and information sharing are therefore care practices crucial to many participants. They have spoken out about how important these practices are to being invested in sex-positive community, and for realizing more full, sexual lives. For instance, education about things such as the modeling of behavioral norms for this sexual 
community is a focal point for consensual participation and interaction. Cooper (2009:113) suggests that the factors that go into creating space that combines "welfare and adventure," or safe, fun sexual adventuring, is one way care is deployed. Through that affective bonds are established and maintained.

KS founder: "I think this is the best sex education there is to see other people having sex. I think it's the best way to expand your understanding of beauty in the world and sexiness in the world is when you can see people that aren't the classic example of beauty or sexiness being beautiful and sexy and be like "Yes, that is fucking hot. I never would have expected that I found that to be hot" (KS founder interview by Zoe Margolis, recording by the author, KS Town Hall London, March 2015).

Former KSNOLA co-producer, volunteer, and participant (20s straight/poly, male, landlord/performing artist/teacher): "I would like to see its popularity grow for that reason because I think more access to these safe spaces where people can explore their interests and feel appreciated and validated is going to change people for the better" (Interview, February 2015).

Furthermore, the norm learning that takes place at KS is part of the way care is facilitated by organizers and reproduced by participants (Cooper, 2009). At the door, event hosts greet and inculcate newbies and long-returning partiers with the Charter, the agreements about what behavior is appropriate, what consent is, and how to give/get it. Remembering that, the politics of play are simultaneously constructed and policed as soon as you walk into a Kinky Salon via such educational tools. People are not allowed inside unless with their PAL, and are made to understand what the whole ideology of 
"distributive accountability" is about. It is these collective agreements of the normative constructs of community that become enforced as the politics that contribute to building a care-based ethos:

\section{Former KSPDX co-producer, volunteer, and participant (30s, bisexual/poly,} female, white, event producer): "I think one of the things I'm trying to do is educate people about what happens if there's a problem with something, like, I've had people not report things. They don't want to cause drama or they didn't, where they felt that someone was going to get kicked out and I'm trying to like educate people that the first thing that we do is not to kick people out. The first thing that we do is just talk to them" (Interview, February 2015).

Many respondents have reported the education component makes them feel safe, welcome, integrated, and like everyone is on the same page as they walk in, insuring a basic set of shared understandings for all participants. This instigates feelings of safety, and an assumption of "looking out for each other," that is interpreted as a practice of care.

\section{Wider sex-positive community member, KS adjacent (20s, queer/poly,}

transgender/nonbinary, white, lawyer and activist): "So I think there are a couple of things, I think one of them is education [...] because I think that there are two types of bad behavior [...] there is harm that happens because of ignorance or because of mistake or because of accident and there is harm that happens because [of] predatory tendencies. And so if you increase the overall level of like consent based confidence in the community and you have a lot more people who are able to communicate compassionately around their needs and on their boundaries they're going to reduce the incidents of accidental or 
unintentional or mistaken boundary crossing, which serves the dual purpose of reducing the overall incidence of the harm" (Interview, February 2015).

Educational events and workshops like Dr. Alison Ash's “TurnON.love” monthly discussion groups are regularly held at the KSSF headquarters (also known as, Mission Control). For instance an upcoming discussion titled: "You don't have to navigate nonmonogamy alone", includes sessions like: "I value (ethical) non-monogamy because...," "Navigating non-monogamy," and "How to pick your person(s)." Education thus serves to reproduce vernacular knowledges that insure safety and reduce harm, developing a collective imaginary reinforced at each point of contact a participant makes with the community.

\section{KSB co-producer, volunteer, and participant (30s, queer, female, European,} educator): "I feel that education and sexual education and all of that is much more of a lifelong thing [...] it's like lifelong learning rather than anything else.

So I am always learning new things about myself, and changing as I you know as you grow up obviously you change as well" (Interview, April 2015).

A final aspect of care through connection I have investigated is established by/for the community through the Internet and social media. It has been argued that the Internet and social media have become integral to understanding contemporary sex culture where sexual expression and sexuality research are being pushed forward by these critical mediums (Ahmed, 2004; Bardzell \& Bardzell, 2010; Garlick, 2011; Ross, 2005). The Kinky Salon community's care work via education and information sharing, promotes outreach and connection with the help of the Internet and social media. As this 
respondent suggests here, for many, online experiences are ubiquitous, informing daily how one moves through and perceives the world:

KS attendee and wider sex-positive community member (30s, straightish, male, black identified, IT marketing and sex-positive play party producer): "for me, being really out about the things I do is also a political thing and so, a political statement $[. .$.$] the more out we are in these communities and with our families$ and with our friends and [...] social circle with whom we feel like we can be sort of safe from major [...] material consequence. The more we're resisting like a two-dollar perm [...] not just monogamy but also the entire hegemony of heteronormativity, right? These ethics are also necessarily extended to the online experience, which is fundamentally where KS is sustained" (Interview, March 2015).

Facebook secret groups are often used by local KS chapters to communicate with people about events and relevant news regarding sex-positive issues: sex work, emotional labor, care, politics, and fun, for instance. By creating a secret Facebook group, all the friends of the local KS chapter's organizers and volunteers can be added to each local group, where their involvement should (presumably) be kept private from their friends and acquaintances that are not in the group. In this way, Facebook secret groups are where people can ideally engage with other event-goers and community members in a publicly private manner, on a well-established social network. Participants can thus find themselves participating in this virtual space as subjects of political liminality - where some ride the fence regarding politics, they are nevertheless political actors involved by participating in the community online. 
With interactive reciprocity and feedback framed also in terms of change-oriented behavior, ${ }^{6}$ oftentimes, this online space is inflected with the act of "lifting others up." This is characterized by the ways community members turn the spotlight on each other to promote work, art, needs, and events. In this way, the KS ethos is idealized and enacted, weaving sexuality into the fabric of this strand of everyday life. Sharing information is an act many participants remarked makes them feel fulfilled in the community, stating that the openness to learn from each other is part of what makes the affective community bond so powerful. This helps support the KS claim that even when sex is not present, people are sexual beings and that influences how people move through the world. So the agenda of pushing forward a new "sex culture revolution" can be understood as a softcore activism even if there is no specific, mobilized campaign partly through these acts of community coalition.

But, can radical acts of being, connection, intimacy, and education actually create sex culture change? Recalling chapter three, I demonstrated that play for some is conceived as transformative. Cooper (2009) raises a question, however, that I think is very pertinent here: “To what extent did sex play itself guide change?" (Cooper, 2009:119). Because remembering that, at Kinky Salon the sex is not supposed to be a focal point:

KSC co-producer, volunteer, and participant (30s, bisexual, male, white, entrepreneur): "I sort of bumped into Kinky Salon somehow and it just won my heart, you could say, for the sex-positive part of it like the whole notion of having sex as being there, a natural possibility of just how we need to relate but not the main agenda [...] And that is the sweet spot I think where Kinky Salon has its 
role. It brings in sex-positive to a playful agenda, it brings in playfulness to the sex show agendas, it brings in an entry point towards more deeper sexual agendas but it brings an entry point for people who have no...it's like a stepping stone" (Interview, April 2015).

Humor, play, and the care found in community, connectivity, and education are therefore the focus, and not the sex play itself that necessitates change. Although, people certainly report exploring and growing in those ways too. This reinforces the argument that it is the care work that is actually driving the "sex culture revolution." Care-based sex play is the threshold, the liminal space through which people move toward choosing their own adventure: be it simply sexual pleasure, connectedness, intimacy, or social change through the radical act of embodied vulnerability.

Former KSNOLA co-producer, volunteer, and participant (20s straight/poly, male, landlord/performing artist/teacher): "I get a lot of pleasure from knowing that people are improved by these experiences. When I see members of Kinky Salon in a state of joy, when they're laughing, when they're happy, when they're comfortable, when I see them expressing themselves, those are really the winning moments for me [...] The real reason that I put energy into this is because I believe its transformative in a positive sense so having experiences that validate that, that remind me that it is working, that's all I need" (Interview, February 2015).

In these ways, the KS community shares in the objectives of a sometimes unspoken sex culture activism. Most respondents just want the freedom to do what they want without the pressure or judgment attached to ethical, consensual lifestyle choices. 
Regardless of sexual or relationship style preferences, respondents overwhelmingly state that the KS community provides, at a minimum, something that can be looked forward to periodically to let loose, play like horny children, get off (or not), and have a good laugh. There is something inherently cathartic in blowing off steam. Many of these respondents desire a self-awareness that consciously evaluates their politics. In doing so they seek to create an energetic foundation for the community that reinforces a utopian ethos. Further reaffirming the KS claim that uninhibited sexuality is revolutionary and good for you.

KS attendee and wider sex-positive community member (30s, straightish, male, black identified, IT marketing and sex-positive play party producer): "So there's [the] transgressive nature of the event and there is a way that I do think that play parties or sex parties are at their best, like a place for sort of personal experimentation, a personal laboratory, you know, a place for personal growth and transformation, you know what I mean?" (Interview, March 2015).

\section{Failures and reconciliations of care: Realizing the impacts of radical acts of being}

Cooper (2007) asserts that such connection and sexual pleasure are linked with nonnormative concepts of care like compassion, service, self-care, being care-free (2007:246). Yet, features of care such as accountability and responsibility are potentially in opposition to the freedoms people assume with sex-positivity. Freedom and liberation are subjective experiences that may be essentialized within sex-positive communities. Furthermore, it is claimed that "hope, fear, joy, shame, boredom, and many other affects may constitute geographies of privilege in addition to or alongside boundaries and regions of inclusion-exclusion" (Twine \& Gardener, 2013:48). It is a privilege to be able to name and own shame. To seek out transcendence of shame is impacted by the 
intersections of sex, race, class, and gender, suggesting that some are in a better position to do such seeking, while others may have to live with it silently. I noted this in conversations with participants who talk about what it means to experience shame. I was struck by the ability some had to acknowledge and articulate it with enough confidence that one won't somehow experience annihilation as a result. These examples also suggest that "the discourse of sex positivity produces a call to action predicated on a repudiation of shame; shame as regressive, ignorant, reactionary and politically suspect" (Mercer, 2018:1305). This can put a lot of undue pressure on people in sex-positive communities to construct an understanding of sexuality through the lens of shame.

Those who verbalize shame are at the same time empowered to do so. I argue that visceral geographies are part of what make such difference apparent, considering more critically the affective geographies of experience: “'Visceral' geography refers to how bodies feel internally in relation with material social space" (Joshi, McCutcheon \& Sweet, 2015:300). Suggesting the reproduction of privilege allows those with it, to be able to name their pain. The visceral nature of affective connection creates different realities for those experiencing internalized feelings that may be denied existence. Particularly in response to the ways such difference actually operates on the lived experiences of bodies marked by difference. Denials of racialized difference in relation to sex and power amounts to microaggressions and "microinvalidation," or, "deligitimazation of the ideas, identity, existence and/or experience of the non-white body” (Joshi, McCutcheon \& Sweet, 2015:305). 
In terms of matrices of power manifest in this community, connection and affect become ingrained as a quality that some say is "hippy dippy," cliquey, or otherwise uncomfortably exclusionary:

\section{KS attendee and wider sex-positive community member (mid 40s, bisexual,} female, white, professor): "I actually think that sometimes the people who work at Kinky Salon are kind of edgy in a way that to me sometimes feels unfriendly and sort of like cliquish in terms of a core group of people who are really out there [...] But then also it feels a little cultish to me that because Ifeel like sexuality is all of ours you know it's in all of us" (Interview, February 2015).

Moreover, cultural creations like a sex culture revolution can be understood as "fictions, individual and collective narratives which we invent to make sense of new circumstances and new possibilities" (Weeks, 1998:46). However, culture creators within the community similarly question the utopia by deconstructing the dominant alternormative discourse, interrogating the romanticized aspects of a liberatory sex-positive culture:

KS participant and volunteer, former KSL co-producer (40s, queer/poly, femme, Caucasian, pornographer/writer/activist): “Well, I mean, a lot of times I feel like my philosophy is sort of against some other philosophy, so it's very reactionary, I guess. I disagree that sex is nice and pleasure is good for you. I think that it can be nice for some people and it can be good for some people in some circumstances. But that it is not the necessary driving force that people consider it and I think that the desire to prioritize it over any other type of intimacy is part of why we have such a problematic relationship with it in the first place. I think that sex can be awesome and explored in lots of different ways and can be 
transformative, can be incredibly healing. But it's also important to remember and acknowledge that, like, sex is also where a lot of our darker prejudices come out and a lot of our media messaging portrays itself. A lot of our desires are formed in part through training on some level. And I think that it's fine to have those desires, but it's really important to critique them and think about them and not just be like, well, that's just my curvy thing, whatever, because it has a greater impact, you know, all of these things are connected" (Interview, February 2015 In response to this statement I am reminded of Lawson (2006): "Social relations of love, connection, mutuality, commitment, and so on are not idealized terrain" and that "we need to take seriously the ways in which social relations are produced through emotion and the ways in which emotional connections are also sites of power" (Lawson, 2007:45). Attitudes about and access to spaces of care, connection, mutuality and intimacy are manipulated by social conditionings and (un)marked by privilege.

One such space of embodied connection amongst participants is located between real-time and a virtual realm of connectedness. The affective spaces created by the KS community simultaneously straddle these two spheres. With the bulk of energy devoted to maintaining community in the nebulous online sphere. Historically, the growth of sex culture and Kinky Salon has been shaped by the growth of social networks:

KS founder: "The social networks were born [and] Kinky Salon was born intertwined with that experience. Kinky Salon could not have existed without social networking because we would have been shut down" (Interview, August 2015). 
But, as I have shown, the Internet as a tool used by alternative sex communities poses a challenge to the development of consent culture due to the poorly reflected privacy regulations that operate based on a largely "non-informed consent culture" (Bechmann, 2014). Even so, the KS Companion manual clearly states the importance of creating and maintaining safe spaces both online and offline. However, over time, as social networks began to have privacy issues, people were being outed and social networks became a potentially dangerous place to be, which has caused a lot of problems for community members. Therefore, in many ways, existing social networks such as Facebook also interrupt the sex culture activism being done by KS. Online spaces pose a risk to the community for increasing privacy concerns and are potentially inaccessible for some. With privacy on social networks constantly shifting, people face dire repercussions as a result of participation in sex-positive lifestyles and communities.

The founder continues: "the privacy issue on social networks is a big problem for people trying to navigate those privacy levels and... [be involved in] something that isn't going to out people non-consensually" (Interview, August 2015). She goes on to say that services like Facebook have surpassed usefulness for communities like KS because everyone is on it, and you do not necessarily "want your grandma to see that you're going to a Kinky Salon" (Interview, August 2015). With the ever-changing privacy policies, it is particularly problematic especially since "real names" issues have limited the ability of people to be "out" online and yet discrete (by choosing another name). Early on this is something that could be done on Facebook by KS community members who were only selectively out about their sexuality. 
KS founder: "When people were being outed [due to Internet privacy complications]. When all these problems started happening with people's lives... Social networking became dangerous because it meant if you were on there it meant being public about who you were sexually it could cause you problems. So people started to do that less, and my ability to reach the friends of friends became less because people were like "I don't want my friends to know what I'm doing on social networks" (Interview, February 2014).

These privacy issues make it impossible for individuals to be " "privately public" ... making connections with many other people, while being relatively private with regard to sharing identity information" (Lange, 2007:372). It was described to me how the site's restrictions on sexual content required all the sex groups to be closed to the general public (i.e. secret groups). But, even the use of secret groups presents a privacy issue for KS community members since people's friends still publicly see (on their newsfeed) when someone joins or likes one of these secret groups.

Wider sex-positive community member, KS adjacent (20s, queer, female, white, commercial and marketing photographer for sex workers): "Whether it's inviteonly or not [...] because people get beat up here a lot and people get, you know...fired from their jobs or whatever. Because if people find out they're too gay or too kinky or too whatever [...] for me, a lot of the time, it's not -- the firing isn't even the worst part. It's the workplace interactions [...] they can make my life really, really miserable until I quit, which basically is what happened. That's how it is for a lot of people in the community here. And so it would have to be very, very secret. But at the same time [...] in a way, that just kind of ... enhances 
that stigma of like we're doing something shameful that we shouldn't be doing" (Interview, February 2015).

Clearly, being outed online has been reported as scary for some individuals who keep aspects of their lives very separate from their families or workplaces. People who identify as poly, trans, queer, and/or kinky, feel vulnerable about their sexuality being open to public scrutiny or debate. But this does not preclude the contemporary reality that "offline physical coupling has in many cases been enhanced by online exchanges" (Ross, 2005:342). Risk, threat, and further stigmatization are some of the tensions inherent in being part of sex-positive communities. Facebook is thus a flawed system for sexpositive community building, nevertheless it is still being used to do so. This underscores the importance placed on safe spaces for sex-positive communities to gather online as well. So, in order to adapt, the KS "Mothership" worked with web developers to create a site that brings all the Kinky Salons local chapters together under one hub. Different than the linked listing on the main KS website, this site is a stand-alone portal for social networking and ticket sales for each location's community and events. Built with the hope of circumventing morality issues and privacy concerns, the aim of this site is to augment the offline experience by being able to view who is going to events and consider with whom one might like to interact.

The KS organization provides its own online resources encouraging community members to become involved by creating opportunities for face-to-face interactions to unfold; all from a simultaneously embodied and disembodied vantage point. However, there is not much discussion about those who cannot easily access the KS community in these ways. For instance, as noted earlier, the KS organization was an early adopter of the 
Internet as a tool for linking and building community, particularly as social networks began to develop: "Kinky Salon was very much a product of Silicon Valley and Bay Area, because when social networking first started... it was something that people in the Bay Area were using" (KS founder interview, August 2015). In the Bay Area then and now, the Internet is often viewed as something that everyone has access to, with a presumption of equal access and availability of resources for all. If the presumption is that most people have the resources to access these online spaces, it demonstrates a lack of awareness that people are potentially being excluded from the community, negatively impacting community-making aims. Because "social exclusion centres upon the processes of unequal access to participation in society" (S. Kenyon et al., 2002:208), the various factors contributing to social exclusion may in fact be a challenge for the KS organization to observe or measure. Embedded in KS operations is the philosophy of inclusivity, but this shows up as a potential blind spot, or failure of care when lack of access to online spaces "render certain bodies and experiences more visible and political than others" (Morrow, Hawkins, and Kern, 2014:531).

The privilege of accessing community, and therefore care, is problematically ingrained into the liberatory aims of sex-positive community. The uncritical assumption of access supports technologies of whiteness that racialize care practices, particularly as care is related through emotion, affect and intimacy. The ways privilege works is revealed to be obscured by/with/in sex-positive vernacular knowledges. As such, sexpositive communities have been critiqued for being very normative white spaces. Some respondents demonstrate this by speaking about being non-monogamous in a very 
unaffected manner. For them, it is already a normalized and unchallenged aspect of their sexual life:

KS attendee (30s, heterosexual/poly, male, Caucasian, writer): "I spent more of my adult life being non-monogamous than I have been monogamous so I don't even know anything else. It plays a role in my life the same way that having pubic hair plays a role in my life. It's there” (Interview, February 2015).

KS attendee (20s, heterosexual/poly, male, white, student): "for me I have freedom and simplicity in my relationships because I don't have all of these weird rules built up around what I can and can't do. All of the other stuff that people think that goes along with polyamory that might be complicated or difficult, that maybe I experienced at some point, but that's been gone too. I feel like for me it's been a lot more easy, simple, and full of freedom than definitely monogamy and other states of being” (Interview, February 2015).

However, the manner in which people of color and queer identified individuals have felt marginalized and had their visibility erased within sex-positive community has often been left unquestioned. In response to this, scholars investigate involvement in kinky, perverted, or alternative sexual lifestyles, looking at the consequences of such alignment, documenting affiliation between polyamory and BDSM, and identifying a reinforcement of homogeneity among class and race (Sheff \& Hammers, 2011). These scholars necessarily demonstrate the need for a better understanding of how people of color navigate in and out of sexual communities. In chapter three I began to demonstrate the complex relationship the KS organization has to issues concerning inclusivity, particularly in how to achieve greater representation in the community. Here, these 
myopic exclusions of sex-positive community are further reflected as being endemic to, "what white folks do" (POC male artist and community organizer, late 30s, NY based, KSNYC volunteer and participant; Interview, January 2015).

This event and community is a product of those who created it, who happen to be white, such that, "phenomenology helps us to show how whiteness is an effect of racialization, which in turn shapes what it is that bodies "can do"' (Ahmed, 2007:150). A further example of this response is also seen as something black Burners have experienced in response to why they would want to spend a week in the desert with a bunch of white folx. As some of my interlocutors shared, it is not necessarily that POC are not interested in sexual communities, but that it comes across as a failure of care (Cooper, 2009) if such communities are not specifically anti-racist in their agendas. For instance scholars of race and BDSM argue that "we must investigate the politics of race, gender, and sexuality as they are intertwined and embodied, enacted and practiced, lived and resisted by practitioners navigating social norms and political rationalities produced in simultaneously discursive and material ways" (Weiss, 2011:184). Therefore, it is also necessary to underscore how transgressive and new explorations in sex/uality are made more complex for people of color given complex histories of violence and oppression, that are sometimes taken up as sexualized fetish (Cruz, 2016). An emphasis is placed on the need to understand the ways sexual liberation movements "marginalize those with various intersections of identities" (Stewart, 2013:2). Who gets to experience liberation, pleasure, shame, care?

By prioritizing these considerations, communities can become more inclusive, "highlighting the potential for people to resist certain oppressions, all while maintaining a 
sex-positive politic inside and outside of their alternative sexual communities" (Stewart, 2013:2). Nevertheless, all the hope and love and positivity cannot erase real-world structural and institutional inequalities. Despite the work KS does to be inclusive, one of the problems with feminist care ethics identified by scholars is "its erasure of the cultural specificity in how care is conceptualized" (Cooper, 2009:106). This erasure may play out as racialized sexual politics in sex-positive play party environments. The affect people of color report feeling is less than welcome, if for no other reason than, "not being chosen," or alternatively, fetishized.

KS volunteer and participant (30s, heterosexual, male, African American, selfemployed creative): "And it's like I would like to see that stigma removed. Why is there an assumption of, like, people into certain things it has to be a result of some trauma, negativity, or blockage [...] And so I think that does influence it somewhat so that there is a stigma within the black community where they're like, "Oh, that's white people do," you know" (Interview, January 2015).

For their part, KS organizers claim to be addressing what it means to be inclusive. There is an emphasis on constantly rallying the community for more input and interaction, with a "ceaseless drive to try better and harder" (Cooper, 2009:114). But this may read as trying to be everything to everyone, which is virtually impossible:

Wider sex-positive community member, KS adjacent (40s, heterosexual, female, white, qualitative healthcare researcher): "Inclusivity I think there's a problem with that. I certainly think there's enough parties out there that if you are gay, bi-, straight, purple, green, blue, whatever, you will find it. But I just don't think in 
this world you have to focus a certain way. You have to be into a certain thing” (Interview, February 2015).

Thus, a "failure of care" can be reflected in blind spots organizers may have around the marginalizing potentials of erasing racialized privileges (Cooper, 2009). The revolutionary vision encapsulated by the KS ethos collides with reality in dealing with the uneven and layered effect of care's power, as is referenced in the quote at the beginning of this chapter.

The organization and participants spend time to work together to refine the ethics carried over into the sex-positive community, however. For instance, the organization has created 'pay it forward' tickets for those who cannot afford event admission (or for those who can), work trades, crafting events, an egalitarian volunteer team structure, open communication, community feedback, and contribution opportunities. Even more, managing power dynamics is often apparent when mediating problems or concerns that arise during events. Most often, a demand is placed upon the volunteer team to address issues around consent and consent violations. Where other events tend to place the onus of responsibility on the person bringing the accusation - which creates a barrier for many to speak up - the KS organization prioritizes these claims. This works to shift responsibility from the person bringing the claim to listening to and understanding what transpired, and working to solve problems through mediation with an immediate response. I have observed how these efforts catalyze a deeper interest in the events and community-making through relational and affective investment, ultimately reproducing an ethics of caring. 
So, what can sex-positive communities do to recover visibility for those it has unwittingly marginalized? To date, the KS organization structures discourse with participants and organizers, to directly reconcile marginalizing blind spots, calling for creating: a set of best practices for welcoming: people of color; lesbian, gay, bisexual and pansexual people; transgender, and gender non-conforming people; fat people (I say "fat" as a way to challenge stigma. It's a description); people with dis/abilities" (from KS organizers' email). By redirecting expectations of receiving emotional and intellectual labor from people of color educating and informing to organizers realizing elisions, is part of the process of coming into alignment with care.

"So rather than expecting people of color to do the work, I said that I need to do the work, I need to do the research, I need to figure out how we talk about these things. I need to learn this stuff myself, I need to make this a priority [...]”" (Founder Interview, June 2018).

Thus, the restructuring of operations to center folx who experience marginalization in this community is a care practice to enact a more clearly anti-racist sex culture revolution. However, there is still a tension that remains that some interlocutors wish for the community to be more, and others are not certain about expecting so much.

\section{KSNYC co-producer, volunteer, and cabaret performer (30s, primarily}

homosexual, male, mixed race, actor): "I'd like the community to be more present more often. I do like the community as a whole. Because that makes it feel special but I want it to have more of a presence so that even those who are excluded are aware of it" (Interview, February 2015). 
Wider sex-positive community member, KS adjacent (30s, bisexual/poly, female, biracial, sex toy peddler): "I would just like to start seeing more reaching out to everyone. I feel like a lot of what's going on is preaching to the choir. We're all talking to each other and it's great, we did it. But it's like we need to start reaching out a little more and grabbing more people" (Interview, February 2015).

Having to take the initiative, and one, recognize the impact of the emotional and intellectual labor being expected from LGBTQ and POC folx in the KS community, and two, redirect that burden, are steps being taken toward anti-racist inclusion strategies. However, according to Doane \& Bonilla-Silva (2003): “Giving up racism means not only abandoning racist attitudes but also relinquishing power and privilege" (2003:251, see also Faria, C., Falola, B. Torres, R. \& Henderson, J., 2019). Stepping aside must be clearly articulated as part of inclusion strategies created to intentionally facilitate a more robust ethics of care.

This is all well and good, but to what end? Why is the mission of "grabbing more people's attention" important to sex-positive community members, and necessary for the social change called for by a sex culture revolution? In a recent follow-up interview with the founder I was able to explore this question. It has been determined that it is not necessary, and maybe not so plausible to try to be everything for everyone. And furthermore, doing so is disingenuous. She discussed with me the personal growth obtained through reflecting in community about the nuances of privilege. Understanding the impact privilege has on a sex-positive worldview is reflected in one so-called ah-ha moment: 
"I realized this whole community is built on white-centric culture [...] I myself am a fan of white-centric culture [...] It's my event. And, I'm white [...] And, I thought, no wonder people of color don't want to come here [...] That realization for me showed me why some people don't feel at home here [...] we don't have the right everything... because it has been white-centric." (Interview, June 2018). So a disconnect is revealed while talking about Kinky Salon, via this bigger idea of what sex culture revolution really is and how it is extended in the KS sex-positive community. At some point, the revolution includes everybody, right?

The way forward has been reported to be about creating a new event under the KS/Mission Control umbrella in San Francisco. With a new team comprised solely of people of color to create, produce, manage, and grow an event specifically for people of color. The "Mothership" is stepping aside, and putting all of the organization's resources and energy into "being of service" to the new team (Founder Interview, June 2018). As the new team forms their own policies they look to build a solid foundation for their growth, suggesting the possibility of limiting attendance only to those that are visibly people of color. I questioned the impact of a colorism policy, and was reassured that that question was asked of the team. Other questions asked of the newly formed team by the founder included: "are you worried about people feeling excluded, because there are going to be people of color who have partners who are white, who won't be able to come? And there are going to be people who identify as POC but they're not. And the guy who is on the team, turned around to me and went, 'They'll get over it'" (Interview, June 2018). All the potential questions and challenges would be left to the team to work out amongst themselves, taking into consideration the specific needs of their community. 
This move is supposed to also help minimize the already present potential to be overwhelmed and uncomfortable at a sex party (which I have felt in my own experience at events):

"the problem with KS not feeling welcoming to people of color is because KS is fucking scary for white people, let alone people of color. If you're a single guy at KS you're going to struggle, whatever the color of your skin. If you're a black single guy at KS you might feel like part of the reason why you're struggling is because people are making assumptions about you because of the color of your skin. But it might not be that. It might just be that you're a single dude and they don't want to talk to you because you're a single dude. So there's all of these barriers to get over in any case. To be able to explore these places is a privilege" (Interview, June 2018).

The objective is to reproduce the care inflected space that enables people to simply get used to KS, on its own terms, uncomplicating it down to its basic complications, recentering sex and play. KS organizers consider this care-oriented approach an "ethical and professional priority" to step out of the way (Faria, C., Falola, B. Torres, R. \& Henderson, J., 2019). By doing so, space becomes accessible to produce an experience unique to KS, yet stripping away the potential extra layers of discomfort and/or racialized (micro)aggressions enfolded into an inherently white-centric space. But there is a particular logic to the reprioritization of Kinky Salon with regard to unifying under what was called a "long-term" strategy for these two events. With this new event coming online that is by and for POC folx, the objective is to plant seeds for "crosspollination" at some point. Then, if there are people that "appreciate 80s synth music 
made by white people, they might come to KS, because they'll understand the space more" (Interview, June 2018). It is perceived that this eventually will be a healthier place from which to relate to others, build community, and participate in sex culture activism. Though it might take some time to observe whether this cross-pollination strategy is even something that the leaders of this new team will want. It may be proven that not striving for an eventual KS monoculture is exactly the point behind the new team's creation.

\section{Conclusion}

The two quotes at the beginning of this chapter highlight the intersections of care and sex that become part of an activist agenda. The productive power of care is put in service of reproducing sex-positive politics that encourage community involvement and the development of culturally specific knowledges. In the liminal spaces created through the Kinky Salon community, alternative lifestyles and sex-positive community become established, organized, and sustainable. All this is approached through the input and interaction of the community. Therefore, in this chapter I have investigated the sociocultural and political impact of what neosexual revolution means for sex-positive community members. Remembering that Woodyer (2012:322) has stated, play can enhance experience to such an extent that generosity of spirit and responsiveness to others encourages an openness that can bring about change. I have shown how such openness is facilitated through strategies of care that are conceived and enacted to provide support. Care is constructed through the practices of this organization as: sex positivity (tolerance, acceptance), creating safe space (online and offline), to encourage connectivity (staying connected to community via social media and offline events), 
education (workshops, playshops, information sharing), and overall community building (socials, mixers). Care is evidenced through the spread of information/knowledge.

People attracted to, or transformed by Kinky Salon have a shared interest or common ground in their personal or professional lives, and often embrace (at least softcore) sexual politics and activisms. It is this type of care that is claimed to change consciousness and contribute to subverting sexual norms. For community members such care-based sex culture activism increases visibility around rights and access to reproductive health care; fighting for the freedom to normalize sex, sexuality, gender identity and non-procreative, consensual choices that privilege autonomy and agency without stigma or legal consequences; the right to choose frequency and type of sexual encounter without being stigmatized; the autonomy to engage in alternormative relationship structures that de-center the heterosexual family unit as organizing principle; encouraging love and care for bodies marked by difference without shame; through an emphasis on creating spaces that encourage connectivity, and overall community-making. However, the radical potentials and problematic slippages in the rhetoric of the KS sexpositive ethos are imperfectly mobilized through community outreach and education. The work to mediate power suggests a response to deconstruct the race and class-based homogeneity that may alienate diverse participation (Sheff \& Hammers, 2010). Striving to mediate privilege while envisioning inclusion politics has come to the forefront of organizing as a result. How accessibility and the privileging of pleasure are then shaped by care practices, prove to be more effective in the aim of actual liberation for all when articulated in anti-racist terms. 
1 ، 'Weak ties' between actors (i.e. loose acquaintances) permit the distribution of information. The circulation of common information (symbols, political information, events, etc.) to loosely connected actors provides them with a common set of signals which allows them to adjust their individual activities in common ways" (Nicholls, 2007: 83). For the KS community, weak ties are the predominant bond through which social networks emerge and are sustained, which then serves to establish a nodal point for organizing and sharing information.

${ }^{2}$ In San Francisco, the birthplace of KS and the location most associated with KS, Carol Queen and Robert Lawrence at the Center for Sex and Culture spend a lot of time working on cases related to sexuality rights. Terrance Allen, who ran the San Francisco Late Night Coalition, became the head of the entertainment commission. They are some of the actors working on making structural and institutional changes regarding sex and the rights of sexual dissidents.

${ }^{3}$ www.kinkysalon.com

${ }^{4}$ https://www.meetup.com/Kinky-Salon-Friends/

${ }^{5}$ https://bayareaopenminds.org/psychotherapists-counselors-member-list/

${ }^{6}$ An undercurrent that extends from the Burning Man principles and KS core principles, discussed in previous chapters. These qualities are inherent in many of the ideological articulations that come from the KS organization and are largely reproduced in the community. 


\section{TRANSNATIONAL INTERROGATIONS OF SEX-POSITIVE CULTURE: Globalizing a privilege of perversities ${ }^{1}$}

"Each city has a doorway to another friend, another community" (From KS newsletter News from the front, party in the back).

"Diasporas of sexual cultures, such as lesbian and gay rights movements, transnational marriage markets, or ideals surrounding sexual freedom, are changing sexual politics within and across nation states" (Howe \& Rigi, 2009:299).

\section{Introduction}

In chapter four, I demonstrated how care-based ethics are enacted via connection, community-making and education. Care ethics rooted in such practices are mobilized by the community as a form of sex culture activism. The outcomes of the KS brand of sexuality activism have been unevenly realized however. For those interested in sexpositive community, the reproduction of privileges reflected through perpetuation of structural inequalities tends to reinforce marginalization that may frustrate realizing aims of sexual liberation. By making reflexive efforts with the community to mediate such power imbalances, care and inclusivity ethics can be refined to come into greater alignment with the needs of those previously excluded from community caringscapes. In response to this however, scholars also caution to "remain alert to these national and international histories, which are embedded in every so-called transnational social movement, regardless of the intention of committed individuals and organizations" (Grewal \& Kaplan, 2001:666).

In this chapter, I take a step back to pay attention to these things, by investigating transnational sex culture and sex-positive community; the flows for which are made possible due to processes of globalization. These processes can be seen as embedded 
within the discourse and observations of how different local chapters reproduce a positive sex culture agenda. Part of what makes an ethnographic study of KS valuable is its positioning as a transnational volunteer organization. This positionality helps me investigate whether "New spaces for politics do not always guarantee the success of the politics, any more than new forms of politics do" (Brown, 1997:188). Therefore, I use the case of Kinky Salon to examine how sex-positive community operates transnationally to explore how discourses of transnational sexuality are informed or disrupted. What I argue overall, and which coalesces in this last chapter is that play encourages affectual investment, inspiring personal change. Personal change lends to community investment through practices of care. Such care-based investment is considered an aspect of "radical acts of being" and therefore, revolutionary by the KS community. That, in turn, inspires transnational spread as people get more involved with the global community (KSGC). Thus the affectual investments engendered by the KS ethos instigates others to take the formula and reproduce it. These investments influence the organization's spread and exemplifies the kind of citizenship transnational KS participants become part of. For instance, Hubbard (2001) suggests, "this alternative model of citizenship is one that cannot be rooted in conventional understandings of public space $[\ldots]$ or private space but in liminal spaces that disrupt dominant geographies of heterosexuality by creating transitory sites for sexual freedom and pleasure where the immoral is moral and the perverse is normal" (Hubbard, 2001:68). I build upon this here by showing how the "global" spread of KS highlights the transnationality of sex-positive cultural activism as it is defined by place, and entangled with sex, class, and race. 


\section{Intellectual setting and methods}

Feminist and geographies of sexualities scholars have touched upon the study of transnational sexuality in a variety of ways looking at: desire, gay marriage, queer politics, lesbian and gay rights, transgender rights and visibility, travel and leisure, globalization, urban and rural sexualities, and sex work. ${ }^{2}$ With more cultural discourse focusing on sexuality, for better or worse, other unconventional sexual practices, like kink, BDSM, and swinging, are also being more openly engaged with on an increasingly global level. ${ }^{3}$ Critical work by queer black geographers like McGlotten (2013), interrogate how alternative sexuality and the urban relate to sex publics. This is interesting to note because the spaces contested in this case are concerned with where and how sex culture is happening. A queer lens is useful then for helping to understand the political economy relationship between sexuality, sexual citizenship, and globalization for LGBTQ rights, "demonstrating the interpenetration and mutual constitution of (local and global) sexual cultures and transnational economic formations" (Gorman-Murray, 2006: 309)

Therefore, the case of Kinky Salon aids me in questioning the spatial negotiations of what sexual subjectivity and citizenship means transnationally within a cohesive, sexpositive community. As I have shown, the sex-positive ethos, when adopted by community members has the effect of causing change, at least at the individual level. Such focus on sex culture change is thus connected to progressive identity politics (Grewal \& Kaplan, 2001). Because of this, scholars are duly beginning to pay "particular attention to the tension between increasingly powerful global discourses and institutions of homosexuality and heterosexuality and between local sexual ideologies and 
subjectivities organized in different, often resistant terms" (Povinelli \& Chauncey, 1999:446). To that end, I have adopted Plummer's $(2001,2011)$ use of "intimate citizenship" to contextualize sex-positive community members concerns regarding their "intimate desires, pleasures and ways of being in the world" (Weeks, 1996:83; see also Woodyer, 2012). Also, bringing Weeks' “sexual communities” (1996), and Plummer's "intimate citizenship," into conversation helps us understand how participants question ideas of morality and control over bodies, emotions, and interpersonal relationships as sexual identity is personally reified.

The Internet as a community space and tool for information sharing on a global scale thus constructs global sexual citizens. Della Porta and Tarrow (2005) agree that "the development of electronic communications and the spread of inexpensive international travel have made it easier for formerly isolated movement actors to communicate and collaborate with one another across borders" (Della Porta \& Tarrow, 7). And Bennett (2005) argues further that communication technologies favor organizational structures that are "loosely linked 'distributed' networks that are minimally dependent on central coordination, leaders, or ideological commitment" (2005:205), adding value to transnational community connections. However, in this case, sex-positive community as a construct and formula is challenged by the complexity revealed in chapter four regarding reproducing a care-based ethos. Transnationally even more so, given the ways that difference, freedom and in/exclusion are defined by people and places.

These scholars suggest it is important that "scholars in lesbian, gay, and queer studies need to think sexuality globally and transnationally" (Povinelli \& Chauncey, 1999:446). With an understanding of space as symbolic and relative rather than fixed and 
absolute - influenced by social relations not excluding sexuality, gender, race, and class (Aitchison, 1999). I was able to observe such forces at work during a transnational tour of Kinky Salon local chapter locations. Therefore, I maintain a focused understanding that transnational flows are always situated within post-colonial contexts. Responding to these calls for greater attention becomes my focus for chapter five. Here I investigate how sex culture is adopted across borders and what that means for cultural transformation discourses widely adopted by sex-positive communities. With this case, I take up the task of expanding upon and contributing to conversations of transnational sexuality studies, investigating sex-positive subjectivities and citizenship. To do so, in this chapter I respond to the following research questions:

Theoretical question: How does sex-positive community operate transnationally? Empirical question: How does the case study of Kinky Salon disrupt or inform discourses of globalizing sexuality and sexual citizenship?

To answer these questions, I investigate how sex-positive community operates across space. In addition to interviews and conversations with community members, the founder and I collaborated on a joint European tour. This tour came at the end of my US local chapter visits (participating in eight of the twelve events established at that time). An invitation for the KS global community's (KSGC) engagement was put out across all the KS Internet channels to announce the tour. The European Kinky Salons aligned their events, with one event a week for three weeks in London, Copenhagen, and Berlin, respectively. That way we were able to country hop, and also get people from other locations to join us along the way. Being able to visit a number of local chapters in the United States and Europe gave me a unique perspective with which to investigate the 
varied ways globalizing sex-positive community operates. During the "Euro tour," many of the Berlin (KSB), Copenhagen (KSC) and London (KSL), and San Francisco (KSSF) core teams attended each other's events. Even more fortunate, we were welcomed to stay with team and community members for each city's event that we visited. We spent a week in each city, which culminated in the respective local KS event. Since we had these generous accommodations (especially for the inquisitive and cash-strapped researcher!) we had hours of informal conversations with team members, volunteers, participants and community members. Predictably, all of this was foremost arranged and managed through the Internet and social media. Being hosted in each person's home provided another unique (if not somewhat biased) opportunity to gain understandings of the local culture, how people live, economics, and sociocultural politics. Our hosts showed us around, introduced us to other community members and talked about cultural politics, particularly related to sex culture, and what that means to them.

These positionalities enabled me to explore how we can understand sexuality in the context of a cohesive, transnational sex-positive community, if the actors at the local scale retain the autonomy to direct and mediate their experiences (Grewal, 2007). And furthermore, interrogate how the organization and community face the possibilities and challenges posed by sex-positive activism that reaches across borders. Through such conversations I can examine how power is structured and reconciled within a sexual community where historico-political realities differently inform organizers' worldviews. Remembering that, "the social aspects of sexuality are always embedded in the material histories of these encounters and must be addressed in nuanced ways" (Grewal \& Kaplan, 2001:671). I show how different local chapters of the KS community handle the pressures 
of chipping away at dominant sociocultural politics that are claimed to limit sexual freedoms. By examining the KS ethos in action at its Global North events, I reveal how a sex-positive community contributes to transnational flows of sexuality, also pointing to the challenges posed by its egalitarian ideals.

\section{Transnational sexual community: Understanding the Kinky Salon Global Community}

Representation, access to space, and opportunities for choice, are all important to securing material needs. It has been argued that pleasure and a healthy sexuality are among these day-to-day needs as well, impacting lives globally. ${ }^{4}$ In order to understand how sex-positive community operates transnationally, I situate the Kinky Salon Global Community (KSGC) as a transnational sex-positive community. The Kinky Salon concept and community is one that has been transplanted to multiple locations over more than fifteen years. Such spread has implications for how care-based sex culture activism expands the influence of how sexual subjectivity and citizenship may be theorized. For instance, this participant articulates the pervasive role played by sociocultural conditionings. These conditionings are widely claimed to be the object of deconstruction for sex-positive community members:

Wider sex-positive community member, KS adjacent (30s, bisexual, female, Cuban American, cosmetologist/professional dancer/sacred sexuality and sex work): "our experiences and our culture and society shape our perspective on sexuality. You know I believe sexuality is often used to control people. You know and I only believe that that's why they try to dissect it out of being a human and human beings are sexual beings right" (Interview, February 2015). 
What responses like this instigated for me were more questions. Has the conceptualization of sexual citizenship changed, and how? If so, how can we understand such changes? And how can we shift theorizations of sexual citizenship now to accommodate global, structural changes? Inspired by Richardson (2018:1257) who suggests a "complex set of new debates and conceptualizations" such as sexual orientation and gender identity rights, and homonormativity, are now important barometers of success in gaining citizenship rights. The context of what it means to be a "good citizen" is also changing (Bell \& Binnie, 2006; Hubbard, 2001). Richardson goes on to argue that sexual citizenship is of course "associated with sexual hegemonies, behaviors and norms, and also informed by, and informs, constructions of race" (Richardson, 2000:126).

I argue that for members of a sex-positive transnational community being a sexual subject (i.e. dissident) is something that pervades everyday life. Kinky Salon community members suggest that the work to create social change is via pushing boundaries that circumscribe what an acceptable sexual subject should be. The reclaiming of citizenry for community members can thus be understood as the creation and building of their transnational community. Furthermore, van Doorn (2013) argues that the relationship between sexuality, intimacy, and citizenship, when invested in by, "a fragile community," (KS founder interview, June 2015; van Doorn, 2013) allows the space for "subsequently developing an understanding of intimacy as a transversal sphere of mutual investment in which political and civic practices can be cultivated" (van Doorn, 2013:157). Such practices can be cultivated by the transnational KS communities being linked by their events, accessible to the sexual citizen that retains the privilege of mobility to cross 
borders, virtually or in real time. In order to build a culture of transnational community linkages, mutuality, reciprocity and accountability, are key. The KS organization encourages it's members to channel their energies into volunteerism, "which is a tactic of survival as much as one of citizenship" (Bell, 1995:149). In doing so, affective investments are built and reinforced, tending to reproduce ideas of belonging to a community. This also sheds light on the rights afforded to those who become members (i.e. sexual citizens).

Questions of sexual citizenship for the KS community are, according to Plummer (2003), a matter of intimacy because citizenship is "concerned with those matters that relate to our most intimate desires, pleasures and ways of being in the world" (Weeks, 1996:83). For KS participants being a sexual dissident with ties to a transnational community provide added benefit. Bell argues (1995:140): "For sexual dissidents, these tensions are manifest in the structuring of all elements of what might be termed a 'sex life' in its broadest $[\ldots]$ excluded at present by prevailing senses of what expressions of sexuality are permissible in public space, and any publicization of their sex lives is likely to attract condemnation or worse." Affirming also what some people I spoke with reported as just wanting the opportunity to not hide their unusual or "perverse" sex life without being called a whore or worse. The benefit of wider community, allows participants to not feel hemmed in by their desires, experiencing the possibility of greater connection. However, reworking the relationship of sex culture to dominant society is one of the KS objectives that is complicated by the different sociocultural and political histories of where respective chapters are located. 
Those who participate and become invested in Kinky Salon's sex-positive agenda are sometimes driven to reproduce these events and communities in different locations. Members of the KSGC often seek each other out to work or play together across locations, which sets the tone for transnational growth. I participated in cross-cultural meetings with European and American producers that recapped and brainstormed new events. Always seeking new ways to do fun things and grow the community, during one such meeting, the group discussed developing an annual summit in the Global South (Bahia and Playa del Carmen, were mentioned); places that have shown interest in creating their own KS events and community - having workshops and socials, inviting the surrounding community. This is an example of how the KS community has followed a trajectory from local to transnational and back again in terms of its growth and cultural flow. For instance, one Berlin organizer affirms that it is precisely the KS ethos that attracts participants:

KSB co-producer, volunteer, and participant (30s, queer, female, European, educator): In my experience, the reason that people get drawn to Kinky Salon in Berlin is because that's what they are looking for [we were discussing sexpositivity]. There are so many sex clubs and like fetish clubs and so much open sex in normal dance clubs in Berlin. But it's very anonymous [...] It's kind of just like faces in the dark [...] The reason that people get drawn to [KSB] is because we do have this idea of like you know you want to be part of a community, you want to know the person, [...] You want to know like maybe what their name is or what they are into or like find them actually stimulating as well, and be like 'oh 
you are really interesting' because you are not just like 'wow you are nude'", (Interview, April 2015).

These comments recall the discussion in chapter three demonstrating the strength of the sex-positive community rhetoric for change-making connection. For some participants, this aspect of the ethos also translates to the event and community being different on a broader scale. I had the opportunity to make note of some of the differences in my observations of various Kinky Salon events and communities. Many of my observations of KS San Francisco are shared elsewhere. The general observations of other events also helped me situate how this sex-positive culture phenomenon is imagined variously. For instance, the New York (KSNYC) local chapter that I at one time volunteered with is organized by a small group of creative people that are very artistically oriented. The aesthetic is edgy and often performers from the Coney Island freak show join the cabaret line up. These events happen regularly, but are generally on the smaller side with as many as 100-120 people attending. Whereas, the New Orleans (KSNOLA) team is run by people involved in the service, art and performance, and sex work sectors. This team has been able to secure sponsorships from liquor and sex toy vendors, an element that most other locations have not reproduced thus far with the exception of London and San Francisco. These events have a strong cabaret and performance flavor due to the creative force of the core volunteer team. The NOLA events are usually produced quarterly and are quite large having close to 200 guests.

Kinky Salon Los Angeles (KSLA) is run efficiently with all the core volunteers filling specific roles. Even though it is a little newer on the scene, this team is well organized and uses a number of strategies to sort each aspect of the event into easily 
identifiable moving parts. Here there is also a strong cabaret and performance element as many of the volunteers are actors, artists, and dancers. These events are produced at least quarterly, and are usually on the large side with anywhere from 150-250 guests. After KSSF, Kinky Salon London (KSL) is the longest running, most well established event. It is produced by a passionate crew, and even more so by many enthusiastic attendees. The core team is one of the larger teams of the KSGC and fiercely egalitarian. It is one of the biggest events with over 300 attendees. Throughout the KSL venue I attended, there were booths and activity stations set up with things like: human whack a mole, fucking machine room, video booth, photo booth, and chill out area. A note from my research diary reads: Party is set up kind of like a Kinky Salon convention.

The Kinky Salon Copenhagen (KSC) event I attended was held at an existing swingers, or "lifestyle," club venue, with an overall attendance of approximately 170 guests. The KSC event theme was "Kinksternational Airport," with the tagline, "Fly me to your dreams" - created to reference the European tour as the theme. This team strives foremost for egalitarianism, is inspired by creative chaos, and is action-oriented. By contrast, the Kinky Salon Berlin (KSB) team is small, bohemian, and focused on DIY, expressive, and performance art. They use an improvisational aesthetic for their organization and event production. The KSB theme was "Naughtical: The Verboaten Party," held in an old rehearsal theater decorated to look like a pirate ship. Sirens and mermaids were part of the entertainment filling different areas of the venue, in character the whole time. These events do not happen regularly with perhaps two, maybe three, events a year being produced. 
The above descriptions help to demonstrate how Kinky Salon is formulaic yet, driven by artful anarchy focused on disrupting norms. The proliferation of the sexpositive ethos and events happens through building momentum around the discourse of sex culture. It spreads from one locality to another in the very definite and at the same time, flexible, terms set forth by the KS formula.

\section{The globalization of sex culture activism}

The organic growth that is often lauded as one of the strengths of the KS community by organizers and participants, demonstrates an opportunity to pay attention to the globalization of sexuality and what this means for sexual dissidents. Are a codified ethics of care, however, as easily translated across transnational sexual community? As I have shown previously, sexual cultures are becoming increasingly popularized, and people are able to come together to engage with intimacy in newer and more accessible ways. Global representations of sexuality and culture are more easily communicated through the Internet and social media. The Internet is thus a tool that helps produce globalized sexual subjects, spaces, and practices, particularly for the KSGC. What makes the transnational spread of the KS sex culture mission viable is the idea that virtual mobility "creates accessibility opportunities, both substituting for physical mobility and enabling access where previously there was an accessibility deficit" (S. Kenyon et al., 213). The Internet then bridges institutional gaps, for those that have access. As I have shown, this is part of the proclaimed strength behind sex culture activism, or revolution in KS terms.

In this case, transnational citizenship and subjectivity for the KS community coalesces into sex culture activism that is established and maintained through communication technologies. Information sharing remains a foremost a tactic used for 
transnational communication of values, learning opportunities, and facilitating outreach and support. For instance, Kinky Salon London has been a leader in creating practices that have been tried and proven effective, instigating new policies for other chapters to model over their eight plus years of hosting regular events. Many of their practices have centered representation efforts that prioritize people of color, differently-abled folx, and new ticketing policies to mitigate class-based exclusions. Their team is keen on sharing their methods with the other Kinky Salons in the global community. This sharing happens online and is first circulated to team volunteers before reaching the wider community. Practices such as these have been effective at building portals for information and virtual mobility where "people can be given a voice and can express their views, gain information and participate in debate without the need for physical attendance" (S. Kenyon et al, 2002:214). Communication is central to the role of transnational expansion and cohesion of the ethos across space. The reification of sex-positive values via virtual mobility creates space for sex culture activism to unfold for transnational community. This in turn encourages growth for communities regardless of physical distance or borders.

Another aspect of strong transnational community cohesion is the volunteers that drive the spread of the event. Remembering that Bell (1995) argues for volunteerism being one of the ways in which sexual dissidents form and reaffirm community as a matter of belonging and survival. Such "radical acts of being," and being of service to community are a source for resistance work being done by community members and organizers. Having a robust and committed volunteer presence is part of the vitality of long-lasting and harmonious local chapters. The emphasis is in on being able to 
thoughtfully engage with each other in productive ways, and ways that will advance creativity. Intra-organization and external communications relay happenings in the KSGC to the local channels through Internet and social media correspondences. Using tools such as Facebook, Instagram, webinars, Google hangouts, teamwork.com, slack, direct opt-in email newsletters, Skype for conducting virtual meetings, and podcasts, organizers reach out to community members sharing information and reinforcing the mission of sex culture revolution. It thus becomes possible for all transnational KS chapters and community members to stay connected. General downloads of current goings-on and event recaps to the community are then claimed to help push growth forward through group process, charting accomplishments, challenges, and innovations. Producers and organizers meet sporadically to discuss what has been taking place in each locale including challenges and successes.

Such meetings are a teaching and learning opportunity for volunteer core team members. This recalls the acts of "lifting others up," discussed in chapter four. To that end, the "Mothership" produces a monthly opt-in subscription email called, "News from the front (party in the back)." In fact, the name of the newsletter was crowd sourced from KS community members, which is one of the ways that the organization actively integrates members' voices into the fabric of KS business. This further reinforces a movement for cohesion and inclusion. The "Mothership" keeps the KSGC engaged further with things like Facebook groups and posts, cultivating an "always there" feeling, where conversation can be sparked on anything from sexual politics like the \#metoo movement, to frivolous pop culture anecdotes. Some KS chapters choose to produce their own local community outreach, so outreach is also decentralized. These examples 
demonstrate how sex culture activism is carried out for the transnational KS community in these acts. In these ways, virtual mobility "has come to refer to activities undertaken via the Internet..." and helps in "creating new and maintaining old social networks online, in virtual communities and networked communities" (S. Kenyon et al, 2002:213). As I explore the transnational aspects of this community, these practices are maintained through care practices invested through collaboration and information sharing.

Such information sharing tools are used by organizers and community members to assist cross-cultural and transnational learning from each local chapter. The goal is said to be in developing best practices, and to encourage community members to be involved with the KS community globally as well. These things amount to an applied praxis of the KS seven core principles, ${ }^{5}$ while maintaining a continual revisionary process aimed at adjusting practices based on community consensus and feedback. Bennett recognizes this "remembering and learning from past events $[\ldots]$ and reporting them back through digital media channels so they can be recognized by activists themselves, as part of larger-scale developments" (Bennett, 2005:205-206). Which, becomes a strong feature of what makes transnational sex culture activism possible for the KSGC. Yet, the tensions between artistic license and oversight of the global community may contradict the creative chaos and deconstruction claims that community members say is a generative force. This is a limitation that I interrogate further.

In response to transnational growth, the KS organization's "Mothership" has begun to direct the affairs of the KSGC as a dedicated facilitator and mentor to both new and established local chapters and communities. ${ }^{6}$ Working to find balance between leadership and egalitarianism, however, is what can complicate or contradict the goals of 
the KSGC. These concerns are among some of the challenges to transnational sexual community and organizing. It is this delicate balance many Kinky Salon chapters strive to achieve in working with decentralized authority, promoting autonomy and participation, while making efforts to dismantle hierarchical relationality in co-production processes. Each team must commit to being proactive to work on mediating inequality, whether by

offering sliding scale ticketing; pay it forward donations for lower income attendees, ${ }^{7}$ and avoiding the use of imagery that is objectifying or discriminatory by embracing and promoting difference in online and offline materials. Structural growth is highlighted by leadership skills that are cultivated among KSGC teams and held up as examples that are working for the respective community, like the KSL team previously mentioned. This is reportedly integral to organic KS growth and change, but also indicative of the challenges of decentralized authority.

\section{What's care got to do with it? Transnational sex culture activism in place}

First and foremost, Kinky Salon is claimed to be a fun, sexy party. The commitment invested into producing sex-positive play parties stimulates the development of community around these events as a focal point. I build from the discussion in chapter four regarding the aim of sociocultural change in attitudes around sexuality, bodies, and pleasure that the KS ethos prioritizes as a global mission. Here I show how transnational sex-positive community members attempt to make sense of what change means. I have previously shown that the feelings of connection and belonging are centrally concerned with safety and care for the KS community. Regardless of sex, the KS domain is conceived of as a "home" for connection, belonging, and potential empowerment. However, the affectual register of empowerment narratives, as seen during past sexual 
revolutions also "highlights the curious absence of considerations of power in debates about sexual empowerment, and argues for the need to think about sexualization in relation to class, 'race', sexuality and other axes of oppression (Gill, 2012:736).

In this project I have been asking who is pleasure, play, shame, or citizenship, good for? I have been investigating whether it is a right to experience liberation by engaging in public forms of sexuality. As I have shown, the power optics of racialization and privilege that become apparent in sex-positive community, including liberatory spaces like Burning Man, makes clear the need to continue to disrupt such discourses by interrogating who gets to do so, and to what consequence? The analogy of drug use in In the Pursuit of Happiness (2018) suggests that what could be experimental for some, is for others dangerous with the potential for extreme punishment. Tensions thus arise particularly around the efforts to mobilize a common set of core ethics into autonomous teams who are guided by communities shaped by different cultural histories. The complex process of constructing absolute truths of what is politically correct then seems to work in opposition to the deconstruction of norms. These tensions are further revealed though my investigation of how these different sociocultural settings impact the realization of a global sex-positive mission. Remembering that for transnational community members, education, information sharing and learning are key to personal development and for spreading the ideas of sex culture revolution.

Currently, in sex-positive community subcultures there are discussions to create change by developing a standardized code of ethics. Within the sex-positive community in NYC, and abroad within the KSGC, issues of consent and safety have come under scrutiny. Consent and safety are among the top concerns for the transnational spread of 
sex-positive culture. For KS participants overall, much of the education that is said to feel transformative contributes to the KS revolutionary flair. To add to the offerings for the transnational community, many of the chapters of the KSGC have also started to host educational workshops with offerings such as: workshops on shibari, or how to negotiate consent during edge play. And the addition of mixers, socials, or even hot tub movie nights are advertised across far reaching channels. These strategies have been credited for effecting transnational community cohesion. For instance, KSNYC and KSNOLA host weekend-long workshops; KSL does an annual hot tub movie social among other regular events and workshops; KSB and KSC have also hosted play workshops, movie screenings, and get-togethers. Kinky Salon SF, London, and NOLA have had great response to their regular socials and workshops for community members to attend and learn from outside guest educators and each other. While I was in New York I had the special privilege to be invited to the home of Betty Dodson with the KS founder. We talked about her orgasmic women's circle workshops that take place in the sunken living room lined with mirrors and an abundance of decorative dildos, where we were presently sitting sipping tea. It was a new and informative experience to speak with a sexually vital 80-something year old queer, female-identified person, who similarly preaches about sexual pleasure being healthy.

KSL co-producer, volunteer, and participant (30s, bisexual/poly, male, white, event producer): "I think the place where people can have learnings and journeys and discover more so, you know, the more straight people can have their views or their experiences challenged by interacting with a [LGBTQ identified person] and be supported through that in, you know, sort of the educational stuffyou do, 
workshops and the information that you send and so on" (Interview, March 2015).

Some respondents mentioned never having even attended a workshop or mixer, yet feel more integrated into the community knowing there are always offerings to learn and connect. Others report feeling excited to be able to attend a Kinky Salon workshop or play event at a different KS when traveling out of town. This is an important point and part of the allure of the global community. Knowing that the community is available to connect is valuable to those who may find their integration into other types of communities complicated by the anxiety surrounding non-normative sexual desires:

Former KSPDX co-producer, volunteer, and participant (40s, bisexual/poly, female, mixed race, social worker): a community kind of opens up a space for people to maybe explore more things, too, that maybe they wouldn't work in a normal situation. I mean, sometimes you'll be, like, at a sex positive event and maybe it's more of a kinky one and you see something happening and you're, like, woah, this is crazy and I kind of want to try that [...] it's getting that space to be where people can really connect with each other and I think it also creates that space where you can ask questions, you can provide education and all that stuff. I think, for me, personally, too, it's also educating yourself on stuff and just really being open" (Interview, January 2015).

Community members remarked that being able to do so on a global scale creates more opportunity for connection with people who share similar interests and already have an interest or investment in a shared community. But for KSL, organizers are more focused on evening the playing field, and deconstructing patriarchy: 
KSL co-producer, volunteer, and participant (30s heteroflexible /bisexual/poly, female, mixed race, sexual health outreach advisor): "Girls make the rules. Girls break the rules kind of thing. It's not about just women being able to set things straight, which is a problem in lots of parties. Women do feel preyed on. Women do feel disempowered at parties. That they are still sex objects and all of this kind of stuff. I understand the mentality of this way of running a party, that women are empowered and they can choose a sex partner and they can decide whether or not they want to play in particular ways and things like that. I really understand that but for me our party, we need to move beyond that. It's not a party just for women to feel empowered; it's a place for everyone to feel empowered" (Interview, March 2015).

Sentiments like these demonstrate that patriarchal oppression denies individual agency and the autonomy to freely explore sexual identity. These perceived limitations affect transnational members of sex-positive community in ways that facilitate and continually renew calls for resistance. Reliance on empowerment and pleasure discourses however, further reveals the power-laden complexities of play, care and connection that may also conceal the intersectional imbalances of power entangled in such liberatory claims.

\section{Challenges and disruptions to liberatory discourses of transnational sex culture}

The peculiarities apparent across transnational chapters in terms of their interpretation of the KS formula, provides insight into how respective chapters also interpret the greater mission of "sex culture revolution." This deeply affects cohesion efforts and the construction of citizenship within transnational sex-positive community. As noted, 
although these locales may be vastly different based on region, it is exactly such differences for each local chapter that often dictates whether events continue and the community thrives. Further demonstrating that the tension between growth and structure can sometimes be equally contentious. A decentralized, hands-off approach is claimed to integrate many voices, provoking such growth organically, by creating space for visibility and sharing to take place. Yet, it is not always an easy or productive road, which sometimes requires tough decisions to be made. For example:

The founder on organizational policing within a hands-off framework: "The problem with Amsterdam [a now defunct chapter, having had their license revoked] is that the imagery that they use for their ads is all female objectification, major sexual objectification... it's like there's no diversity, there's no queerness there's no humor there's no personality. It's basically a fetish club, what they're doing is throwing a fetish club with the Charter and the PAL system and they have a playspace but apparently no one goes in there... and they have a cabaret but again, it's all skinny white women taking their clothes off. These cultures are really different... maybe that's ok... but then I have people coming to KSSF from Amsterdam and they are saying ... they don't get it, that's not a KS..." (Interview, February 2014). ${ }^{8}$

This begins to demonstrate the often complex and layered differences that emerge when negotiating a sex-positive, chaos driven ethos in practice. The KS ethos is troubled as it rubs up against each team's autonomy to enact the formula with its own spin. These contradictions are instructive for understanding the antagonisms concealed within the promotion of transnational sex culture. Enacting the formula is thus influenced by the 
personal and sociocultural politics of each team's members, and by extension, their attendant community. As I have pointed out, the affectual investments made by community members is suggestive of how sex-positive community is proliferated. The allure of community is strong for many. And such investments in community often leads to personal fulfillment and perhaps taking up a more hands-on role. Newcomers and long-standing community members alike may feel compelled to volunteer as a core team member as a result. However, the organization and community's own institutional structure becomes more complicated and potentially contradictory with globalizing flows of sex culture. This troubles the affect, activism and fun that is supposed to be the point of being involved in the first place.

Community members involved with local chapters of KS must negotiate the pressures of transforming sociocultural politics by listening to and interpreting their community's needs. As a volunteer co-producer, the experience taught me how important it was for our team to aim for enough internal cohesion to then be able to listen to and interpret the needs of the community. For me, that consisted of spending time with the team to understand allegations of rape and other privacy violations of the wider sexpositive play party community in our "neighborhood," which by extension also affected our community. Given that there is a lot of overlap between different communities and events, it is crucial to take into consideration what community members say about people and situations. What it took to manage those situations required more help and input than I could have anticipated. Making it even more challenging is the task of getting the basic operation's needs met. The internal, team-based struggles sometimes experienced as part of investing in the KS community suggests that producing a KS event is not for everyone. 
As a volunteer core team member, I can say that it was an intense experience on several levels. In particular, for core volunteers there is a concealed emotional toll that is taken by being invested in producing a KS and reproducing sex-positive sexual politics. This can for some become a burden of emotional and physical labor that goes unpaid and sometimes unacknowledged by community participants. In my time as a volunteer coproducer, I experienced feelings of frustration and anxiety; after all, it is still event production, combined with pretty heavy politics coupled with the responsibility for others' safety and fun time. People with little understanding or regard for politics, whether sexual or care-based, are less likely to remain invested (or do well by their teammates). For my experience, group dynamics within the core team were challenging at points when lack of communication and consensus, would have in my opinion, favored a bit of leadership or management to keep things on track. Deciding on anything as a team seemed to take forever, frustrating results that sometimes fell flat or were simply unsuccessful. It is likely that these outcomes had a lot to do with a lack of comfort I had relinquishing control, as a control-oriented person. This was a great learning experience that showed me that orientations such as I had are what become off-putting to other team members, causing team cohesion to break down. As a result, teammate responsiveness, event production and community growth became unbearably slow. When teams face internal struggles such as this, team cohesion begins to fail and things can easily fall apart with people losing interest or leaving the team.

Transnationally, these struggles also play out contingent to local institutional structures that may constrain teams economically, socially, or ideologically. For instance, how easy is it to get a venue that will be cool with sex and alcohol comingling on site? 
Not at all an easy task. What are the local laws for having sex and alcohol consumption if it is a "private" party? This takes some conscientious digging in to local legal frameworks to be able to maintain invisibility underground from state surveillance and at the same time be somewhat in alignment with laws so the community is not put in jeopardy. These initial challenges can be intractable for local chapter teams. But at the end of the day, the founder has said, and others would agree, "if you're not having fun, stop doing it" (numerous conversations to me, 2014-2015).

In any case, a common theme expressed by many producers is that believing in the mission and the type of unique vibe that KS events and community represents, does not preclude the kind of stress and frustration that may arise while working to dismantle hierarchies within internal structures. Moreover, the level of interest and interaction from the community is directly related to frequency of outreach, and relates to the community's overall resonance with the local core team. There have been several instances where local teams have interpersonal conflict and managerial incongruities (for those who are not used to egalitarian, decentralized anti-hierarchies, and how to get projects done when there is no discernible management). These frictions have affected the cohesion of community and created a breakdown in local chapters, in a couple cases entirely shutting down. This is another indication that involvement in sex-positive community and organization is laden with emotional labor emphasizing the need for a commitment rooted in wanting to spread the sex-positive culture message.

Unusual divergences from the formula are then both a strength and weakness, when it comes to the "Mothership" making final determinations about what is in alignment with the KS ethos overall. One such divergence from the formula was 
observed with the KSC team. We met the team who was comprised of a large board of mostly new people. Before the tour, the team had not communicated with the "Mothership" that they created a board with many members as the structure for the team. The "Mothership" questioned this new type of team arrangement, but became eager to understand how that team's structure is implemented and whether it was working. ${ }^{9}$ This team was also trying out something new by not having a designated playspace during their event. Instead, they said this was done to get the guests in tune with their surroundings and co-create a space, requiring attention be focused on checking in and being more interactive with each other. My first impression was that this experiment was not totally successful because in my experience, knowing where to go to play helps keep safety and boundaries in place, and help one relax amidst the chaos. Although the team and the guests seemed to like it. But a divergence from the creation of intentional spaces for instance, calls into question the ability to safely have fun. It is thought that creating such varied spaces, rather than a spatial free-for-all, reinforces a commitment to not having sex be the focus of the event, the idea being to normalize it further. They stated their objective was to inspire people to relate to each other in unconventional ways, where a space does not influence one's behavior - an example of the logic that appears contradictory, yet lauded as part of the ways the KS formula evolves and changes, especially in response to the needs of the community.

Another example of how divergence from the model can incite transnational praxis to change was observed during the KSB event. During the event, volunteers appeared less committed to their duties and it did not really seem like anyone was "on" working the event after coming through the door. The participants seemed to enjoy the 
carefree vibe, even the bar volunteers were letting people behind the bar to serve themselves, which is not really "supposed" to happen. I did not observe any walkaround hosts, what I believed to be one of the key ingredients to the event formula, and one of the ways the safe container is thought to be created and held. There was no "Chartering" 10 at the door, rather the KSB team went through the Charter as part of the cabaret beginning. The audience was well captivated and participated in the beginning of the cabaret with the Charter. However, I questioned this move, because for instance, the cabaret happens an hour or two after the event starts so people are welcome to engage with one another prior to the (cabaret) the KSB Charter. It worked well for this event and community, but in other circumstances could potentially be problematic where safety is concerned. This restructuring is contentious because one of the objectives is to stop people as they come in the door, slow them down, and make sure everyone knows the rules upon entering. I asked the "Mothership" if this was something that needed to be discussed, but it was decided if it works for them and people are engaged and on the same page, then that is their own spin on the formula.

For many transnational organizers, the freedom to interpret the KS ethos is part of what keeps people engaged. For many, such freedom is also implied within the ideological structure of "sex-positivity" and creative chaos. Each local chapter I visited in the United States and Europe have diverged from the KS model somewhat. Such innovation is conceived as growth, which is ultimately encouraged as part of the organic process the KS organization imagines for the community. This may pose a problem though, when measured by an unclear litmus regarding what is an acceptable reproduction of norms and values, aligned with care practices, and also policing and 
oversight. It was thus informative to observe how individuals with different cultural values revised the formula yet remained in alignment with a culture of belief that adopts and intertwines with the existing KS formula. In doing so, these teams instigate a revision to the formula suitable for their particular community needs. These are some of the particular ways that sociocultural differences complicate the proliferation of transnational sexuality with particular regard to this case.

To this end, there have been many discussions among the KSGC organizers around issues of power, ways to deconstruct hierarchies and structural imbalances, especially regarding race and class. It is an issue that confounds each local chapter differently based on each unique set of cultural and social contexts. Organizers possessing non-violent communication skills and understandings of discrimination have proven to be in better dialogue with their communities, able to better adjust decisionmaking practices. The tensions regarding race and class discussed in chapter four are also troubling to the transnational objectives of sex-positive community. Such contestations get at the difference of each community being informed by sociocultural and historical settings, feeding back local politics that may disrupt the continuity of the SCR mission within transnational operations. This is an important point because at one event on the Euro Kinky tour there were instances of pejorative racial stereotypes (i.e. blatant racism) in costuming and the creation of event materials. One of the core team members dressed as a terrorist and did not understand the racial aggression in this. On more than one occasion interlocutors (both core team volunteers and community members) remarked that race is not a problem in their country. They claim there is no racial tension, discrimination, or inequalities among people. During conversations with team members it 
was remarked that cultural norms privilege a fundamentally egalitarian way of life, built into the cultural fabric, and paying capital to those who "don't stand out" too much. However, this is not the whole story. Due to historical-cultural constructs, privilege, and the pervasive dominance of whiteness in the group, these organizers did not understand racial inequality as an issue, nor recognize the inappropriateness and aggression in costume and language choices.

In response to which there was a long, somewhat heated discussion about race and context. While we were in Europe we had many conversations with global team members from KSSF, KSL, KSB, and KSC about social politics, particularly about racism and inequality in light of what was witnessed. The "Mothership" wanted to take the opportunity to meet with all the core team members of the European Kinky Salons, especially having been to each event by this time. Conversations in the meeting were concentrated on racism, because of the cultural sensitivity that KS tries to enforce, and the pushback related to the organizer's terrorist costume. Discussions also focused on cultural appropriation, class/economics, and accessibility (as in for differently-abled participants). Remembering from chapter three, "Microinvalidation refers to the questioning or deligitimization of the ideas, identity, existence and/or experience of the non-white body" (Joshi, McCutcheon \& Sweet, 2015:305), some discussants were dismissive and even impatient (like, we over here do not have race problems and you are bringing your issues to the table where we have none), regarding the race focused debate. Even more bypassing, in this post-event meeting, some team members were more keen to ask how to encourage more LGBTQ involvement, than say POC involvement, especially since they receive few complaints about lack of diversity or inclusivity. Some teams are 
more intent on pushing boundaries in what they prioritize as creative chaos (implied as the objective of art). They asked this discussion group about how to go about breaking taboos and challenging people constructively. How can this be done without causing offense?

KSC co-producer, participant and volunteer (30s, primarily heterosexual, male, Caucasian, artist) goes on to say: "Our mindset is we want to fuck the pope in the ass with a strap-on, but that's the point [...] I realized that we are bunch of, I don't know, assholes, freaks in that sense that we are not here to be political correct. We are actually here to push the boundaries and fuck with the world [...] That's the most important part of Kinky Salon Copenhagen [...] And that's where the energy comes from, but it's of course done in a way that is caring and playful and with self-irony" (Interview, April 2015).

The discussion group affirmed that, in general, a "salon" is a place where people come together and share ideas and that sometimes discomfort results from constructive growth - but offense should not be included. In these conversations with transnational team members, it was strongly reiterated and emphasized to remember the KS core principles: "community spirited, creative, sexually progressive, and socially conscious." The suggestion being that no matter where teams are located, they should be able to create without these ethics being undermined. The strengthening of cohesion around vernacular knowledges was importantly reinforced through a strong reminder that for the local chapter agreements it is important to avoid: "racism, colonialism, oppression, cultural appropriation, and marginalization." These concepts are considered to be 
objective truths relating to colonial histories, which may still be understood from various transnational perspectives as not sexually progressive or liberatory.

To reiterate the quote used at the beginning of this chapter, these reflections point to ways "diasporas of sexual cultures, such as lesbian and gay rights movements, transnational marriage markets, or ideals surrounding sexual freedom, are changing sexual politics within and across nation states" (Howe \& Rigi, 2009:299). A critical example of this is found by exploring how these complex racial and sexual politics are handled within transnational sex-positive community. Complications arise in the enactment of anti-discrimination policies across transnational KS locations. In concert with such needs, extended conversations between transnational KSGC team members and the "Mothership" continue to revolve around how to embody policies that are inclusive by anti-racism standards. Prompted in part by a woman of color KS team member, the "Mothership" has been called out in an attempt to reconcile how these objectives should evolve for the organization and community. Called out for "not doing it right," regarding representation, it is claimed that changes being made by the organization to become aware of and rectify marginalization and exclusions have not been specifically anti-racist enough, although anti-discriminatory in tone. It was explained that anything less than anti-racist policies contribute to the persistence of microaggressions and othering within the KS community, and by extension, out into the world. This does undermine the effectiveness of the so-called "sex culture revolution," which, in this case, and the cases of the sexual revolutions of the past, are often born from the worldview of "white habitus". Such positionality is a socialization process that "conditions" and reinscribes "taste, perceptions, feelings, and emotions on racial matters" (Bonilla-Silva, 2017:152). 
Racial privilege that is occluded by colorblind liberalism conceals the uneven socialization processes which feedback into maintaining such habitus.

Currently, new strategies are being worked out. Recalling from chapter four, new events by and for POC are coming online with the support and resources of the "Mothership," and a commitment to stepping aside, noting: "one of the downsides of the strategy of allowing teams to really have autonomy and control over their own events, is that when there is conflict or disagreement, or if [...] they're out of alignment, then it's not as simple as getting back on track... (people are not fired and solutions often take time to work out, my addition)... it's not that simple" (Interview, June 2018). That begs the question then, what does being out of alignment entail? And who makes the final determination? The above example demonstrates one approach, that despite the contradictions of egalitarian objectives, inclusion requires "also relinquishing power and privilege" (Doane \& Bonilla-Silva, 2003:251) to produce a shift in the discourse made by stepping back to center marginalized voices.

For other KS chapters, there is a clearer focus on intersectional sexual politics. I have observed KSL, KSNOLA, KSS, and KSNYC, to be among those teams increasingly conscious of critically analyzing outreach and language to facilitate greater inclusivity and accessibility - with the goal of better serving the community and creating safe spaces for exploration. The KSL team is at the forefront with KSSF, leading the way for other teams to explore their influence for setting the tone to evolve policies and structures that continually evaluate and question their social impact. This is something that various chapters are either actively asking or cultivating in welcoming materials and community 
spaces. For instance, KSNOLA is keen to use clear, inclusive language that is unambiguous:

\section{Former KSNOLA co-producer, volunteer, and participant (20s straight/poly,} male, landlord/performing artist/teacher): "We use language in our advertisement. We call it a safe space. We say queer friendly. Amongst other tags so we really try and give shout outs to the different communities and say this party is for you, on Facebook even on our physical fliers by having really queer entertainment" (Interview, February 2015).

Overall, these transnational community debriefs provide clarity regarding contextualizing histories and sociocultural politics, and how integrating those understandings into the ways $\mathrm{KS}$ is variously created by the global teams plays out.

Although troubles such as these have not been uncommon for KSGC teams, with more teams getting started there have been revisions to the oversight of transnational local chapters. Intentional oversight attempts to reclaim care practices in the form of team visits and meetings, with mediation sometimes required to problem solve. However, as I have noted when these stresses become too burdensome, teams dissolve and communities fail. During the course of fieldwork and writing, I have observed the dissolution of three different KS local chapters, ${ }^{11}$ effectively having their creative commons licenses revoked. But it is reported that by staying true to the mission of playful sex and care-based community, many challenges are at least met by the time the event starts. Even still, what I observed, particularly in comparison during the Euro Kinky tour, is the motivation to instill more progressive inclusion politics evenly across locales, despite taking a largely hands-off approach to management. Repeatedly while speaking with respondents from all 
locations I have been made aware of the value attributed to the creation of safe, welcoming space. Part of the strength claimed to be behind striving create welcoming and inclusive space is summed up in this quote:

KSL co-producer, volunteer, and participant (30s, bisexual/poly, male, white, event producer): "I think the value is [...] creating a place where people who are not satisfied with the existing options, you know, gay parties, kinky parties, straight sex parties could meet and connect and find a home, find a place where they belong. And the second thing is I think somewhere where people whose view would otherwise be excluded or marginalized in society [...] can find and sort of effectively can feel that it is a safer space..." (Interview, March 2015).

Nevertheless, as I have shown, not everything can or will be suitable for everyone: KSL co-producer, volunteer, and participant (30s, bisexual/poly, male, white, event producer) continues: "I think we need to be more accepting with people who have, for example, identified as asexual or demi-sexual, you know, it isn't a lot of people, but I think we should be sensitive with the kind of [...] you know, we need to sort of do as much as we can to take [that] into [account] because we have some really bad feelings [...] excluding people [...] they don't want to be exposed to these fuck fest" (Interview, March 2015).

How the transnational sex-positive KS organization and community envisions, versus manages their sex culture activism despite different spatial contexts, is a task largely left up to each local team. To interpret their community's needs they invest in community participation and feedback. Time will tell whether creative evolution and revisionary 
strategies will be effective for long-term sustainability and growth of the global community.

\section{Conclusion}

Kinky Salon as an organization expands globally by engaging with and encouraging participants who embody a sex-positive ethos. Some participants become motivated by a deeper investment to throw sex parties that are a platform for resistance. This unusual type of sexuality activism although transnational in scope, is one where sexuality, agency, and power are prioritized through a politics of the body, oriented to care and community-making across space. It is thought that playful sexual expression that seeks to push boundaries both in terms of personal exploration and cultural norms, will stimulate social and cultural change. Although the transnational KS locales are vastly different based on region, it is exactly such differences for each local chapter that often dictates whether events are sustained, and the community developing or growing according to its own collective needs. The ethos and formula are interpreted based on the personal politics of each location's production team. How difference is regarded, embraced, or erased, becomes even more apparent among examples of the transnational local chapters. Thus, the manner in which the organization and community spreads its ethos is demonstrative of what Grewal \& Kaplan (2001) signal as "the demise or irrelevance of the nation-state" (2001:664). However, the evidence points to how structural oppressions are also subject to processes of globalization. Globalization of systemic oppressions may be reproduced as race and class based inequities transnationally, further highlighting how failures of care can subvert the mission to be sexually liberating for all. What this

demonstrates is how the construction of sexual subjectivity and sexual citizenship ${ }^{12}$ may 
be shaped by spatial negotiations of what these things mean transnationally, and how that is complicated within a subjectively cohesive community.

These snapshots illuminate the impact of investments made by participants to nurture the oftentimes, "fragile community," through practices of care I discussed in chapter four. What remains a constant theme is the claim that the organization and community maintain a collective commitment of working toward transforming sex culture, globally. Historical and changing cultural, social, and political ideologies are thus pushed back against by local teams, redefining radical potentials of what was previously circumscribed by embedded political particularities of each place. By critically engaging with the transnational community to create representative space, it is hoped that events will cross-pollinate over time. However, a lack of "engagement with power" (Haritaworn, Lin \& Klesse, 2006:519) to instigate institutional policy change, and differences in social consciousness and connectedness to sexual politics complicate this task, particularly unevenly from Europe to the US. Drawing from Puar (2003, 2005), it is believed that such moves are made by the organization to create a framework for globalizing sexuality that makes efforts to center queer, anti-racist, feminist politics across borders. ${ }^{13}$ In that case it will be necessary for organizers to better understand such variances. This work therefore attends to the calls scholars have made to contextualize transnational flows of sexuality within an increasingly globalized world.

\footnotetext{
${ }^{1}$ Sheff, E., \& Hammers, C. (2011). The privilege of perversities: race, class and education among polyamorists and kinksters. Psychology \& Sexuality, 2(3), 198-223.

2 See: Binnie, 2004; Boellstorff, 1999; Cefai, 2013; Fisher, 2014; Hoad, 1999; Kempadoo, 1998; Kulick, 1999; Larvie, 1999; Phillips, R, 1999; Rofel, 1999.
} 
${ }^{3}$ For instance, Napier University, Edinburgh has created a curriculum for an 'alternative sexual practices' class (http://www.mirror.co.uk/news/uk-news/nipple-pinchingdominatrix-hired-university-10804359.

${ }^{4}$ Barker, Gill \& Harvey, 2018; Cooper, 2007; Richardson, 2000; Weiss, 2011.

${ }^{5}$ Playful, safe(r), inclusive, creative, community spirited, socially conscious, sexually progressive (https://www.kinkysalon.com/the-7-core-values-of-kinky-salon/).

${ }^{6}$ This has developed from times past in which new and existing teams were left more to themselves to organize and produce. With problems among some local chapters struggling to survive, in addition to applications for new locations, the founder shifted to become more present in whatever way each particular community may need (with limitations).

${ }^{7}$ Pay it forward tickets are priced at a higher cost to subsidize those who purchase lowincome tickets. The community members "who have it" are encouraged to "give it" when they can.

${ }^{8}$ By the time I started fieldwork the Amsterdam team had already been put on notice and had their creative commons license revoked.

${ }^{9}$ Incidentally, it never was revealed to us clearly why the structure of a board is successful or useful, though the team liked it and said it was working. My takeaway was that because many people wanted to be involved it became a way of integrating people based on level of commitment. Those people committed to being on the board had different responsibilities toward event production and community interaction than say, other volunteers that helped out the day of the event. Being on the board was about level of investment however, and for instance, the team would always say that to be part of the core team everyone must clean the toilets.

${ }^{10}$ The act of stopping people as they enter, making them slow down, read aloud and agree to the Charter, or agreements of proper behavior.

${ }^{11} \mathrm{KS}$ Amsterdam was on its way out as I began data collection, mostly for the reasons shared in the quote above, and for continually being talked with about these objections without making any changes. Prior to starting event production volunteering with KSNYC, it was produced by another person. That license was revoked because a number of important formulaic things were not being followed and volunteers were reportedly mistreated. A new team stepped up about a year later. Finally KSPDX was recently put on permanent hiatus. 
${ }^{12}$ For sexual subjectivity and citizenship, see: Bell, 1995a; Bell \& Binnie, 2006; Binnie, 1997a; Grewal \& Kaplan, 2001; Herman, 2007; Richardson, 1998, 2000; Staeheli, Kofman \& Peake, 2004; Weeks, 1998.

${ }^{13}$ (Petzen, 2012; Puar, Rushbrook, Schein, 2003; Puar, 2005). 


\section{CONCLUSION: \\ Learning from failures: \\ Deconstructing privilege to create a more caring agent of chaos}

May 2018: "WHO takes BDSM and fetishism off the sick list. The World Health Organization (WHO) follows the Nordic countries in repealing sexual minorities with consenting practices from the International Classification of Diseases in the new revision ICD-11. This is a milestone in the work for human rights and sexual liberation, says Ingvild Endestad, leader of FRI, the Norwegian LGBT organisation for sexual and gender diversity. Consensual sexuality has nothing to do with psychiatry. This is an immensely important recognition of the sexual diversity among us, she says."1

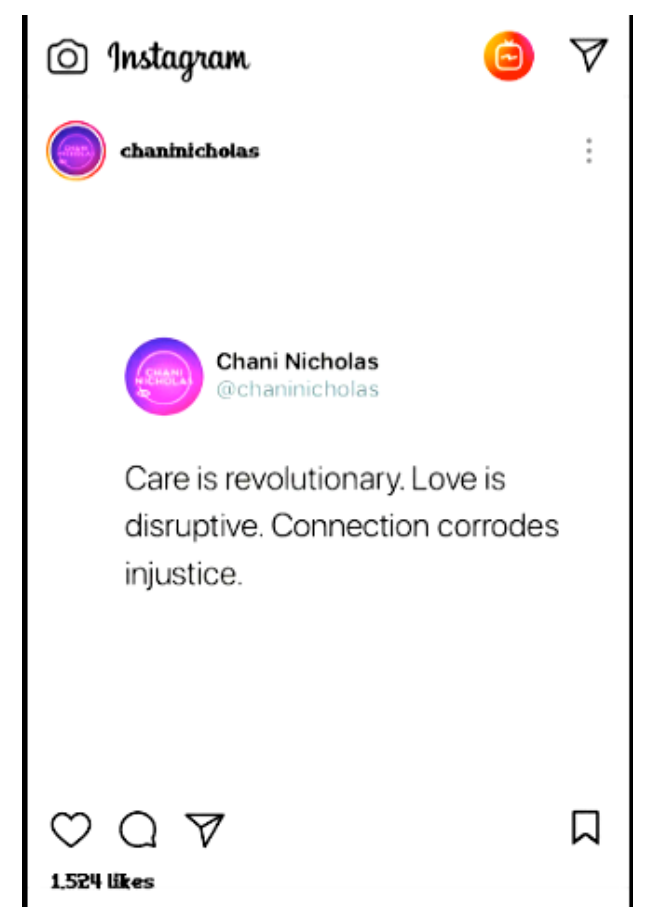

Chani Nicholas, Astrologer ${ }^{2}-J_{u n e} 2018$

\section{Introduction}

These two excerpts are distinct in their origins. The first is from the world of politics and sexuality activism. The other a pop-culture astrologer. Yet each central to the argument of this dissertation. In the above excerpt, dissident sexualities and practices are legitimized by an international health organization. This intervention by WHO suggests 
that sexuality is still viewed as being in need of liberation. Lack of sexual freedom is evident in such revisions to outdated morality laws. Also demonstrating that sexual freedom is unevenly experienced among people who are precariously living at multiple intersections of structural and institutional bias and oppression. What these things also show is that there is cultural capital invested in the productive potential and emotional labor of care work - to ground the human rights inherent in sexuality rights. This idea of care is not simply intellectual, but something that is being lobbied for in day-to-day popular and political culture. The current political climate in the United States is one in which violence and apathy seem to be common reactions to increasingly untenable and oppressive institutional directives. At the same time, people continue efforts to organize, call others out and in, take ownership and responsibility for behavior, vocalizing and agitating for change. This is emotional and intellectual labor as care work at its most mercurial; unpredictable and ready to fight for the belief in a better, more just life free to be. But these ideas are themselves rather utopian, aren't they?

I approached sex-positive play parties and communities as spaces that at first glance seem very cohesive and unflinchingly forward thinking. Many of the ideologies that are said to anchor a sex-positive ethos are things that I "totally get"... affect, intimacy, emotion, care, communication, consent, and inclusivity. I was moved by the good feelings expressed to me when I went to events and talked to participants. Then, the data reveals another story about the complex messiness of living, such that sociocultural and intellectual perspectives may be enhanced. For this community, being a sexual dissident registers differently for each participant. Some feel like it is no big deal and they want to go to a fun party and have sex. Others express the liberated feeling of going 
home to their chosen family. And still others have felt excluded or marginalized. Some of the challenges faced by sex-positive community are in overcoming structural and institutional facades that seem to be crumbling under the weight of inevitable change. And, to show up for the microinvalidated, and nuanced struggles, listening and stepping aside to figure out the work of integrated, non-violent sociocultural transformation.

In this dissertation I have worked with the case of Kinky Salon to argue that sexual play is political and sex-positive culture important to pay attention to. It is transformative for many of its participants, and such potentials for transformation are guided by an ethics of care. Care work is conceived as acceptance, creation of publicly private safe space, information sharing, education, community-making and support, which suggests sexual exploration may unfold. Organizers and community members work together to create, deconstruct and recreate an ethos of inclusivity for sexual liberation to be accessible to all. Seeking the fulfillment of liberatory potentials is articulated as sex culture activism. The community is thus able to spread beyond borders with a transnational reach, proliferated with the help of the Internet and social media. Now, feminist geographers are exploring how technologies are changing the dynamics between users and sexuality especially through social inscription and performativity (Bardzell \& Bardzell, 2010). In response to moving the framework of neosexual revolution forward, scholars have been working to understand the impact the Internet has on social theory and sexuality particularly with regard to intimacy, as a new arena for experience, exploration, and experimentation (Ross, 2005). These new ways of looking at sexual subjectivity through the lens of the Internet could shed light on failures of the past. However, failures of care make invisible those who are marginalized and excluded. Thus, 
the development of transnational sexual community is complicated by push back from those that argue the organization doesn't do enough to embody anti-racist inclusion strategies.

Using a mixed methodological, ethnographic approach I have shown how sex is spatial, play is political, and care tactics constructed as revolutionary. I investigated eight of the twelve locations operating at the time of data collection to do this work. Consequently, transnational methods as an analytical frame (Massey, 2010; Datta, 2016) become necessary in order to engage with data on the KS community across the United States and Europe. I first began in the United States, where for a year I spent as part of a small Kinky Salon New York (KSNYC) volunteer core team. This position helped me delve into the inner workings of KS, learning what it takes to produce an event, and myself having to negotiate the pressures of articulating a sex-positive, boundary-pushing agenda collectively. By doing so, I had a unique vantage point to investigate how the organization and community variously function as mechanisms for change via what organizers and participants term a "sex culture revolution". And I show how this case is instructive for understanding claims that a new turn in an ongoing sexual revolution is articulated through the Internet and social media. I demonstrate that such Internet and social media interventions impact bodies and connections, laden with both the challenges and the promise of changing cultural and social norms. I reveal what sex culture activism looks like for global community members and how that activism is variously deployed. In doing so, I have underscored the challenges and possibilities the community faces by investing in a playful, sex(y) party that happens across borders. Also, exploring how that objective can achieve (or cannot) such idealistic goals. 


\section{Reviewing the findings: Terrible, wonderful utopias}

Feminist and geographers of sexuality have investigated how sex and the urban are bound up, where ideologies and movements of sexual revolution have often been centered. Sex in public, public sex, and sex publics, are contextualized as a critical part of understanding sexuality and the urban (McGlotten, 2013). Furthermore, queer culture building emphasizes safe zones (or building what KS considers a "safe container") where possibilities for change merge with ideas that heteronormativity is not the default for sex culture (Berlant \& Warner, 1998; Castiglia, 2000). The spaces created by the KS community are always public and private at the same time, underground yet constructed as intentionally welcoming. This duality frustrates efforts to be accessible and inclusive. However, the Internet as a tool for dissemination of information, education, and a hub for gathering is relied upon to model behavior, brand the "sex culture revolution" (SCR), and circulate sex-positive vernacular knowledge. I have demonstrated how the KS ethos is reproduced and operates across space, and how sexual subjectivity and citizenship are shaped by spatial negotiations of what dissidence means transnationally.

Methodology

Post-field notes, June 2018: What at first began as a project with which I had very little personal connection, five years later has served as a liminal space, through which I have come to understand self-care. In many ways, moving through this work has been an ebb and flow of anxieties, building of strength and understanding, then falling again to higher ground still, requiring I get up and find a way forward. During this project one of the many things that I was conscious of, even throughout this analysis and writing phase, was the question of 
changing the field and being changed by the field. Essentially, deciding when to stay, when to go, and how much to give have also directed the energies put into this work. With that said, I have questioned: What have I achieved? What have I learned? How did I contribute? Did I do right by this community, and also maintain unbiased intellectual rigour? Where is my/the value in the finished product? Will it be good enough? What is good enough? The literal closing of a chapter, the end of these years-long work. Now what?

In the methodology chapter I examine what it means to be a researcher and subject to sexualization in the field. I use autoethnographic reflections, like the above to demonstrate the personal, conflicted nature of conducting sexuality research. Furthermore, during the write up, I have embraced a slow scholarship. ${ }^{3}$ It is articulated as a means for resistance against neoliberal university structures that tend to devalue such efforts, and as a means for survival. I find this type of scholarship personally exciting because it helps me feel less less than, about taking what I consider a really long time to finish this work. And so, slow scholarship provides a little validation that this contribution is relevant to academia. With a commitment to radical vulnerability and situating positionality autoethnographically - and with great support from my patient advisors, and all the participants who trusted me and this process, this piece of knowledge could be produced. I feel like I have contributed well to feminist, ethnographic methodologies in these respects.

In chapter three I discuss the KS mission of "bringing sex out of the shadows". Here I provide a narrative of the lived experience of sexual dissidents that has only been attempted so far by LGBTQ scholars by exploring sexual play and politics. For the 
organization and community members, this is thought of as revolutionary and sought after through an emphasis on play, safety, and connection. The data illustrates that the affectual tone that the KS events curate with humor and the absurd, make resistance and nonconformist behavior accessible to even participants who would not necessarily consider their actions to be revolutionary. Distinguishing the difference between this sexpositive sex party and mainstream events is something that was revealed as very important to KS participants. This serves to highlight the play, care, and community aspects of KS as necessary for achieving the aim of weaving sexuality into daily life. For the KSGC it is the autonomy to be left alone, yet have the benefits of services such that citizens not identified as "pervert" might receive. Furthermore, the liberal use of the term "pervert" for the KS community is done as a critical reclaiming to indicate a positive identity that has non-conforming sexual tastes and practices. I have found that given alternatives, some of my interlocutors discuss doing their part to subvert normative structures by engaging in non-conformist behavior, to spur changes in attitudes around sexuality despite stigma and the often persistent need to closet behavior. I demonstrate that a by-product of participation in communal, sexual play is something that people reported feels transformative, shifting their worldview and prompting personal growth. The sense of support derived from an investment in a sexual community is what may prompt sustained engagement with sexual politics. Thus, the illusion of communitas figures strongly for understanding the response to KS events that conceal ordinary-reality and status-identities, such that people are able to relate to each other in new and creative ways. The integral nature of the Internet and social media to these objectives further highlights how this case contributes to increasing attention paid to the globalizing 
sexualization of culture. I also reveal that despite an emphasis on inclusivity, the KS event and community is viewed by some as being less than welcoming, and a space that reproduces exclusionary privileges and/or marginalization. Therefore, feminist and sexuality scholars that are invested in understanding the productive potential of play and care may find value in this case.

In chapter four I show that the sexuality activism in which the organization and community engages in is a care-based approach. Here, I center care as a new way of constructing sexuality activism. I argue that in this revised neosexual revolution, community members are bound together through affective bonds made and emotional labor to insure care for each other, which is distinct from sexual play. For the "citizenpervert" then, the development of a "third space" is realized by creating "a space of oscillation, perhaps, between the public and private; always moving, redefining” (Bell, 1995:147). Care practices for the organization and community are thus understood as the sometimes uneven, reworking of accessibility and inclusion ethics; and the creation of safe spaces for play, education, and community-making. New ways of organizing, dismantling, and creating structures around identity politics force sociocultural and political shifts in many cases. Embracing a healthy sexuality as part of daily life is at the heart of the guiding principles that Kinky Salon models and reproduces. But these principles can get swept up in an idealism that is also unconscious to its own structural shortcomings. For many, participating in this event and community is a privilege, allowing for experimentation, and the restructuring of non-normative relationships. And for many, it is an engagement laden with the potential for being confronted with trauma, risk, fear, and social exclusion. This demonstrates a failure of care, and blind spot to the 
layers of structural inequalities people may have to engage with to have the privilege of recreating sexual identity. The work to simply increase inclusivity without prioritizing representation reveal further failures of care that marginalize people of color and LGBTQ individuals. The Internet and social media enable and constrain these engagements as well. These tools are revealed to be the primary hub for maintaining community cohesion. However, with access being an assumption, and uncertain privacy adding to risk, these tools are still heavily relied upon for information sharing and educational resources. Therefore, questions are raised about the limitations of a revolutionary vision. Yet, restructuring toward anti-racist, class-conscious policies is guided by new voices integral to the KS sex-positive agenda. This work underscores the productive and problematic ways transformation is conceived of in connection with others, to sociocultural politics, activism, and sexuality. This is important to note because it demonstrates that small, quotidian acts of care contain the potential for academic and cultural transformation to become possible.

Finally, chapter five takes a step back to situate sex-positive community transnationally. Here, I answer the call to investigate sexuality transnationally, contributing to expanding discourses on transnational sexuality and activism. I do so by showing how people construct their subjectivity around sexual identities that are fundamental to how they engage with the world, and why this matters. The case of Kinky Salon is one among burgeoning transnational and globalizing sexuality discourses. Through this case I have been able to investigate its revolutionary mission and how that is prioritized for the various transnational chapters. I identified that the sex-positive ethos and formula is unevenly reproduced across space. And I further explored how that 
impacts the formation and cohesion of sexual community transnationally. I argue that sexual dissidents in the transnational KS community demonstrate new ways of crafting an intimate citizenship that defines desire, attachment, and how one engages with the world.

By touring many Kinky Salons in the United States and Europe, I more closely investigated the global reach of transnational sex-positive community, underscoring the variance in how agendas are conceived and influenced by diverse histories. I reveal what sex culture activism looks like for community members transnationally and how that activism is variously deployed. In doing so, I find complications arise around racial exclusion, marginalization, and aggression, which are further troubled by the idealism of egalitarianism and hands-off management. Some problems are resolved through group process, discussion, debate, and consensus about founding principles. However, more recent interventions toward an anti-racist, inclusive agenda have required a commitment to stepping aside.

\section{Contributions and Interventions}

Overall, this is a focused view of a subject that offers many avenues for intervention. With this dissertation I have sought to reveal that the work to create change is happening around us in ways that require we look differently to understand differently. My research on Kinky Salon and their vision of sex culture revolution is politically significant to what globalization of sexuality scholars suggest, that we pay attention to how transnational sexuality operates (Grewal \& Kaplan, 2001). I extend this work by engaging with Weeks' (1996) sexual community, extending also, how a sexual community can be conceived, creating sexual diasporas (Howe \& Rigi, 2009). The proliferation of sex culture and alternative possibilities for intimacy thus happens more easily via the Internet and social 
media (Bardzell \& Bardzell, 2010). Such spatial negotiations are complicated by the constructing and remaking of identity, and over time are influenced by globalizing sexuality increasingly now accessible virtually and otherwise.

This research thus provides an interdisciplinary and feminist exploration of the intersections of embodied sexuality, play, care, and the influence of the Internet on understandings of transnational sexuality that pushes forward geographies of sexualities scholarship. With this work I contribute new ways to theorize care and intimacy via sexual relationships. I contribute to ongoing discourses regarding sexual dissidents. And, I expand upon existing work by showing how dissidents create their diaspora from a shared cultural construct of sex-positive, liberatory sexuality, and the problems inherent in such strivings. I do so to contribute new understandings to feminist and geographies of sexualities and activism by centering care ethics as activist-oriented, mobilized through a sexual community that has transnational reach. The process of this work turned out to affect me personally as well. I have contributed to the field of sexuality studies and geographies of sexualities by becoming entrenched in a very taboo place, the effects of which have been seen at multiple scales. Therefore, in the methodology discussion I emphasize the intellectual necessity of transparency, reflexively situating the researcher, with a commitment to radical vulnerability and situating positionality autoethnographically. In doing so, I add texture to the ways a feminist, ethnographic lens is valuable to interdisciplinary studies.

\section{Avenues for future research}

The result of how I have attempted to theorize sex parties and sex-positive community via $\mathrm{KS}$ in all its complexity, suggests new ways of understanding subjectivity and sex 
culture. This is only one view of an event, organization, community and its participants. But it is a view that tells a particular story based on the concrete data collected. It has been more than three years from the end of data collection to the completion of this manuscript. In that time sex-positive culture and communities have changed. However, this subject provides many fertile resources for continued writing and productive scholarship to unfold. Some questions that have emerged for potential future research suggest more deeply digging into "place" attached to transnational iterations of sex culture. A comparative analysis of the sociohistorical and political structures of one place versus another organized around each site and informing those relationships, can provide more understanding in terms of an historical to contemporary trajectory of analysis for each location, further enhancing studies of transnational sexuality. Research that more deeply explores trauma and transformation, interrogating the relationship between trauma and the pathologizing of sexuality and how these things are worked through, is an equally exciting new avenue this research may take. This would also enable a closer look at how these transformation narratives then link to larger society, underscoring further how the personal and political are co-constituted. And importantly, black feminist work like that of Audre Lorde, Marlon Bailey, Dána-Ain Davis, Shaka McGlotten, and Jennifer Nash can assist me in more deeply refining analysis of sexual liberation and transformation narratives as they are related to race, sex, power and the erotic.

\footnotetext{
1 "The withdrawal of fetishism, fetishistic transvestism, and sadomasochism as mental illnesses can lead to the same pride and freedom that other queer groups enjoy. The revision of the F65 ICD chapter can also make it easier to encourage research, get rid of anti BDSM laws and become included in national laws against discrimination, Endestad concludes" (http://revisef65.net/2018/05/27/bdsm-fetishism-sadomasochism-globalmilestone-human-rights-reform).
} 


\section{${ }^{2}$ http://chaninicholas.com}

${ }^{3}$ For slow scholarship, see: Mountz, A., Bonds, A., Mansfield, B., Loyd, J., Hyndman, J., Walton-Roberts, M., ... \& Curran, W. (2015). For slow scholarship: A feminist politics of resistance through collective action in the neoliberal university. ACME: an international E-journal for critical geographies, 14(4). 


\section{REFERENCES}

Aguilar, J. (2012). "Situational Sexual Behaviors: The Ideological Work of Moving toward Polyamory in Communal Living Groups." Journal Of Contemporary Ethnography, 42(1), 104-129.

Ahmed, S. (2004). "Collective feelings: Or, the impressions left by others." Theory, Culture \& Society, 21(2), 25-42.

Ahmed, S. (2007). A phenomenology of whiteness. Feminist theory, 8(2), 149-168.

Ahmed, S. (2014). Cultural politics of emotion. Edinburgh University Press.

Aitchison, Cara (1999) "New cultural geographies: the spatiality of leisure, gender and sexuality” Leisure Studies, vol. 18(1): 19-39.

Albert, S. (2011). "Spontaneous pleasures: Sex between women in public places." Sexualities, 14(6), 669-680.

Allyn, David. (2000) Make Love, Not War - The Sexual Revolution: An Unfettered History. Routledge, London and New York.

Allyn, D. (2016). Make love, not war: the sexual revolution: an unfettered history. Routledge.

Altman, Dennis (2002) Global Sex. University of Chicago Press.

Angelides, S. (2012). "The 'Second Sexual Revolution', Moral Panic, and the Evasion of Teenage Sexual Subjectivity.” Women's History Review, 21(5), 831-847.

Atkinson, S., Lawson, V., \& Wiles, J. (2011). "Care of the body: spaces of practice." Social \& cultural geography, 12(6), 563-572.

Attwood, F. (2006). "Sexed up: Theorizing the sexualization of culture." Sexualities, 9(1), 77-94.

Attwood, F. (Ed.). (2014). Mainstreaming sex: The sexualization of Western culture. IB Tauris.

Bailey, B. (2002). Sexuality and the movements for sexual liberation. A companion to post-1945 America, 260-276.

Bain, A. L., and C. J. Nash. (2006). "Undressing the Researcher: Feminism, Embodiment and Sexuality at a Queer Bathhouse Event." Area 38 (1): 99-106. 
Baldassari, E. (2016). "The Kinkification of the East Bay." East Bay Express. Retrieved from http://m.eastbayexpress.com/oakland/the-kinkification-of-the-eastbay/Content?oid $=4665086$

Bardzell, S., \& Bardzell, J. (2010). "Mapping Techno-Sexuality through Feminist Geography: Inscription, Performativity, and Paradoxical Space." Presentation, CHI, Atlanta, GA (April 10-15, 2010).

Barker, M; Langdridge, D., Eds. (2010) Understanding Non-Monogamies. Routledge

Barker, M. (2013). "Consent is a grey area? A comparison of understandings of consent in Fifty Shades of Grey and on the BDSM blogosphere." Sexualities, 16(8), 896-914.

Barker, M.; Gill, R.; Harvey, L. (2018). Mediated intimacy: Sex advice in media culture. Sexualities, 1363460718770449.

Barnhill, A. (2011). "Bringing the Body Back to Sexual Ethics.” Hypatia, 28(1), 1-17.

Beasley, C., \& Bacchi, C. (2007). Envisaging a new politics for an ethical future: Beyond trust, care and generosity - towards an ethic of social flesh'. Feminist Theory, 8(3), 279298.

Bechmann, A. (2014). "Non-Informed Consent Cultures: Privacy Policies and App Contracts on Facebook." Journal Of Media Business Studies, 11(1), 21-38.

Bedfellows, D. (Ed.). (1996). Policing public sex: Queer politics and the future of AIDS activism.

Bell, D. (1995). "Pleasure and danger: the paradoxical spaces of sexual citizenship." Political Geography, 14(2), 139-153.

Bell, D., \& Binnie, J. (2006). “Geographies of sexual citizenship.” Political Geography, 25(8), 869-873.

Bell, D., Binnie, J., Cream, J., \& Valentine, G. (1994). "All hyped up and no place to go.” Gender, Place and Culture: A Journal of Feminist Geography, 1(1), 31-47.

Bennett (2005) "Social Movements beyond Borders: Organization, Communication, and Political Capacity in Two Eras of Transnational Activism" in Transnational Processes and Social Activism. Eds. Della Porta, D; Tarrow, S. Rowman \& Littlefield.

Berlant, L., \& Warner, M. (1998). “Sex in Public.” Critical Inquiry, 24(2), 547-566.

Beres, M. (2007). “'Spontaneous' Sexual Consent: An Analysis of Sexual Consent Literature." Feminism \& Psychology, 17(1), 93-108. 
Bigger, S. (2009). "Victor Turner, liminality, and cultural performance." Journal of Beliefs \& Values, 30(2), 209-212.

Binnie, J. (1997). "Invisible Europeans: sexual citizenship in the New Europe." Environment and Planning A, 29(2), 237-248.

Binnie, J. (2004). The globalization of sexuality. Sage.

Binnie, J., \& Valentine, G. (1999). "Geographies of sexuality-a review of progress." Progress in human geography, 23(2), 175-187.

Blasius, M. (Ed.). (2001). Sexual identities, queer politics. Princeton University Press.

Boellstorff, T. D. (1999). The perfect path: Gay men, marriage, Indonesia.

Bondi, L. (2003). "Empathy and Identification: Conceptual Resources for Feminist Fieldwork." ACME: An International E-Journal For Critical Geographies, 2(1), 64-76.

Bonilla-Silva, E. (2017). Racism without racists: Color-blind racism and the persistence of racial inequality in America. Rowman \& Littlefield.

Bosco, F. (2007) "Emotions that Build Networks: Geographies of Two Human Rights Movements in Argentina and Beyond." Tijdschrift voor Economische en Sociale Geografie. 98, 5: 545-563.

Boyce, Paul, M. Huang Soo Lee, Carol Jenkins, Salim Mohamed, Cheryl Overs, Vera Paiva, Elizabeth Reid, Michael Tan, and Peter Aggleton. (2007). Putting sexuality (back) into HIV/AIDS: Issues, theory and practice. Global Public Health, 2(1), 1-34.

Breitbart, M. M. (2010). Participatory Research Methods 11. Key methods in geography, 141.

Briggs, L., McCormick, G., \& Way, J. T. (2008). "Transnationalism: A category of analysis.” American Quarterly, 60(3), 625-648.

Brown, M. P. (1997). Replacing citizenship: AIDS activism and radical democracy. Guilford Press.

Brown, G. (2007). "Mutinous eruptions: autonomous spaces of radical queer activism." Environment and Planning A, 39(11), 2685-2698.

Browne, Kath; Lim, Jason; Brown, Gavin. (2009). Geographies of Sexualities: theory, practices and politics. Ashgate, Burlington, VT. 
Billo, E., \& Hiemstra, N. (2013). "Mediating messiness: expanding ideas of flexibility, reflexivity, and embodiment in fieldwork." Gender, Place \& Culture, 20(3), 313-328.

Browne, Kath. (2003) "Negotiations and Fieldworkings: Friendship and Feminist Research." ACME: An International E-Journal for Critical Geographers 2 (1): 132-146.

Burke, N. (2014). "Positionality and pornography." Porn Studies, 1(1-2), 71-74.

Burkett, M., \& Hamilton, K. (2012). "Postfeminist sexual agency: Young women's negotiations of sexual consent.” Sexualities, 15(7), 815-833.

Cahill, C. (2007). The personal is political: Developing new subjectivities through participatory action research. Gender, place and culture, 14(3), 267-292.

Califia, P. (2000). Public sex: The culture of radical sex. Cleis Press.

Castiglia, C. (2000). “Sex Panics, Sex Publics, Sex Memories.” Boundary 2, 27(2), 149175.

Cefai, S. (2013). "Introduction: cartographies of belonging: the marketisation of desire through media, practice and place." Gender, Place \& Culture, 21(5), 582-588.

Chapkis, Wendy. (1997). Live Sex Acts: Women Performing Erotic Labor. Taylor \& Francis.

Chrisman, Robert (Fall 2013) "A Critique of the Sexual Revolution" The Black Scholar v. 43, no. 3, pp. 29-31. Reprinted from: The Black Scholar, Vol. 9, No. 7, Blacks \& the Sexual Revolution (April 1978).

Clement, G. (2018). Care, autonomy, and justice: Feminism and the ethic of care. Routledge.

Comella, L. (2014). “Studying porn cultures.” Porn Studies, 1(1-2), 64-70.

Conradson, D., \& McKay, D. (2007). Translocal subjectivities: mobility, connection, emotion. Mobilities, 2(2), 167-174.

Cook, Elaine. (2005). "Commitment in polyamory." Electronic Journal of Human Sexuality 8 Academic OneFile. Web. 4 Feb. 2016.

Cooper, D. (2007). "Well, you go there to get off' Visiting feminist care ethics through a women's bathhouse." Feminist Theory, 8(3), 243-262. 
Cooper, D. (2009). "Caring for Sex and the Power of Attentive Action: Governance, Drama, and Conflict in Building a Queer Feminist Bathhouse." Signs: Journal Of Women In Culture And Society, 35(1), 105-130.

Cover, R. (2014). "Sexual Ethics, Masculinity and Mutual Vulnerability." Australian Feminist Studies, 29(82), 435-451.

Crang, M., \& Cook, I. (2007). Doing ethnographies. Sage.

Cruz, A. (2016). The color of kink: Black women, BDSM, and pornography. NYU Press.

Cuomo, D., \& Massaro, V. (2014). "Boundary-making in feminist research: new methodologies for 'intimate insiders'." Gender, Place \& Culture, 23(1), 94-106.

Cupples, J. (2002). "The field as a landscape of desire: sex and sexuality in geographical fieldwork." Area, 34(4), 382-390.

Cvetkovich, A. (2003). An archive of feelings: Trauma, sexuality, and lesbian public cultures. Duke University Press.

Cvetkovich, A. (2007). "Public feelings.” South Atlantic Quarterly, 106(3), 459-468.

Datta, A. (2016). Translocal geographies: spaces, places, connections. Routledge.

Datta, A., \& Lund, R. (2018). Mothering, mentoring and journeys towards inspiring spaces. Emotion, Space and Society, 26, 64-71.

Davidson, Dr Joyce; Smith, Mick, Bondi, Liz, Eds. (2012). Emotional Geographies. Ashgate Publishing, Ltd., 2012

Davis, E. C. (2009). "Situating "FLUIDITY" (Trans) Gender Identification and the Regulation of Gender Diversity." GLQ: A Journal of Lesbian and Gay Studies, 15(1), 97130.

Del Casino, V., \& Brooks, C. (2014). "Talking about bodies online: Viagra, YouTube , and the politics of public(ized) sexualities." Gender, Place \& Culture, 22(4), 474-493.

Della Porta, D; Tarrow, S. (2005) "Transnational Processes and Social Activism: An Introduction" in Transnational Processes and Social Activism. Rowman \& Littlefield.

Diprose, G., Thomas, A. C., \& Rushton, R. (2013). "Desiring more: complicating understandings of sexuality in research processes." Area, 45(3), 292-298.

Dixon, D., \& Straughan, E. (2010). "Geographies of Touch/Touched by Geography." Geography Compass, 4(5), 449-459. 
Doane, A. W., \& Bonilla-Silva, E. (Eds.). (2003). White out: The continuing significance of racism. Psychology Press.

Downing, L. (2004). "On the limits of sexual ethics: The phenomenology of autassassinophilia." Sexuality and Culture, 8(1), 3-17.

Downing, L., Langridge, D., \& Barker, M. (2007). Safe, Sane, and Consensual: Contemporary Perspectives on Sadomasochism.

Edensor, T., \& Bowdler, C. (2015). "Site-specific dance: revealing and contesting the ludic qualities, everyday rhythms, and embodied habits of place." Environment and Planning A, 47(3), 709-726.

Ellingson, L. (2006). “Embodied Knowledge: Writing Researchers' Bodies Into Qualitative Health Research.” Qualitative Health Research, 16(2), 298-310.

England, K. V. (1994). “Getting personal: Reflexivity, positionality, and feminist research.” The Professional Geographer, 46(1), 80-89.

Escoffier, J. (1998). The invention of safer sex: Vernacular knowledge, gay politics and HIV prevention. Berkeley Journal of Sociology, 1-30.

Escoffier, J. (2008). The Sexual Revolution, 1960-1980. GLBTQ Social Sciences, 1-7.

Esteban, M. J. (2009). Cruising Utopia: The Then and There of Queer Futurity. New York: New York UP.

Evans, S. M., Gosse, V., \& Moser, R. (2003). The World the 60s Made: Politics and Culture in Recent America.

Fahs, B. (2014). '“Freedom to' and 'freedom from': A new vision for sex-positive politics." Sexualities, 17(3), 267-290.

Falzon, M. A. (2016). Introduction: Multi-sited ethnography: Theory, praxis and locality in contemporary research. In Multi-sited ethnography (pp. 15-38). Routledge.

Faria, C., Falola, B. Torres, R. \& Henderson, J. (2019). A long way to go: collective paths to racial justice in Geography. Professional Geographer. Accepted.

Faria, C., \& Mollett, S. (2014). "Critical feminist reflexivity and the politics of whiteness in the 'field'." Gender, Place \& Culture, 23(1), 79-93.

Fisher, K. (2014). "Positionality, subjectivity, and race in transnational and transcultural geographical research.” Gender, Place \& Culture, 22(4), 456-473. 
Fisher, B., \& Tronto, J. (1990). Toward a feminist theory of caring. Circles of care: Work and identity in women's lives, 35-62.

Fox, C. O., \& Ore, T. E. (2010). "(Un) Covering Normalized Gender and Race Subjectivities in LGBT" Safe Spaces".” Feminist Studies, 36(3), 629-649.

Frank, K. (2003). G-strings and sympathy: Strip club regulars and male desire. Duke University Press.

Frank, K. (2007). “Thinking Critically about Strip Club Research.” Sexualities 10 (4): 501-17.

Garlick, Steve. (2011). “A New Sexual Revolution? Critical Theory, Pornography, and the Internet." Canadian Review of Sociology/Revue canadienne de sociologie, Vol.48(3), pp.221-239

Gedalof, I. (2000). "Power, Politics and Performativity: Some Comments on Elisa Glick's 'Sex Positive'." Feminist review, 64(1), 49-52.

Giddens, A. (2013). The transformation of intimacy: Sexuality, love and eroticism in modern societies. John Wiley \& Sons.

Gill, R. (2012). "Media, empowerment and the 'sexualization of culture' debates." Sex Roles, 66(11), 736-745.

Glickman, Charlie. (2000). "The Language of Sex Positivity.” Loving More 21 www.lovemore.com

Graham, N. (2014). "Polyamory: A call for increased mental health professional awareness." Archives of sexual behavior, 43(6), 1031-1034.

Green, M., \& Lawson, V. (2011). "Recentring care: interrogating the commodification of care.” Social \& Cultural Geography, 12(6), 639-654.

Grewal, I; Kaplan, C. (2001) "Global Identities: Theorizing Transnational Studies of Sexuality” GLQ: A Journal of Lesbian and Gay Studies 7:4 pp. 663-679

Grewal, I. (2007). "Understanding "global community" in cultural studies."

Communication and Critical/Cultural Studies, 4(3), 332-335.

Grov, C., Parsons, J. T., \& Bimbi, D. S. (2007). "Sexual risk behavior and venues for meeting sex partners: an intercept survey of gay and bisexual men in LA and NYC." AIDS and Behavior, 11(6), 915-926. 
Gorman-Murray, A. (2006). "The Globalization of Sexuality." Cultural Geographies 13 (2): 308-9.

Gorman-Murray, A. (2007). "Sexy stories-using autobiography in geographies of sexuality." Qualitative Research Journal, 7(1), 3-25.

Greenwood, J., \& Guner, N. (2010). "Social change: The sexual revolution."

International Economic Review, 51(4), 893-923. http://pareto.uab.es/nguner/gg-ier.pdf

Greiner, C., \& Sakdapolrak, P. (2013). "Translocality: Concepts, applications and emerging research perspectives." Geography Compass, 7(5), 373-384.

Grunt-Mejer, K., \& Campbell, C. (2015). "Around Consensual Nonmonogamies: Assessing Attitudes Toward Nonexclusive Relationships." The Journal Of Sex Research, 53(1), 45-53.

Halkitis, P. N., Parsons, J. T., \& Wilton, L. (2003). "Barebacking among gay and bisexual men in New York City: Explanations for the emergence of intentional unsafe behavior." Archives of sexual behavior, 32(4), 351-357.

Hamer, Diane Ellen (2014) "Legacies of the Sexual Revolution" The Gay \& Lesbian Review v. 21, no. 5, pp. 17-18.

Harden, K. (2014). "A Sex-Positive Framework for Research on Adolescent Sexuality." Perspectives On Psychological Science, 9(5), 455-469.

Haritaworn, J., Lin, C., \& Klesse, C. (2006). "Poly/logue: A Critical Introduction to Polyamory." Sexualities, 9(5), 515-529.

Hayes-Conroy, J., \& Hayes-Conroy, A. (2010). "Visceral geographies: Mattering, relating, and defying." Geography compass, 4(9), 1273-1283.

Heap, C. (2008). Slumming: sexual and racial encounters in American nightlife, 18851940. University of Chicago Press.

Heise, T. (2009). "Degenerate Sex and the City: Djuna Barnes's Urban Underworld." Twentieth-Century Literature, 55.3, 287-321.

Herman, R. D. K. (2017). Playing with restraints: space, citizenship and BDSM. In Geographies of sexualities (pp. 103-114). Routledge.

Herzog, Dagmar (2008). Sex in Crisis: The New Sexual Revolution and the Future of American Politics. Basic Books, New York. 
Hoad, N. (1999). "Between the white man's burden and the white man's disease: Tracking lesbian and gay human rights in Southern Africa." GLQ: A Journal of Lesbian and Gay Studies, 5(4), 559-584.

Houlbrook, M. (2001). "Toward a Historical Geography of Sexuality." Journal Of Urban History, 27(4), 497-504.

Howe, C., \& Rigi, J. (2009). "Transnationalizing desire: Sexualizing culture and commodifying sexualities.” Ethnos, 74(3), 297-306.

Hubbard, P. (2001). "Sex zones: Intimacy, citizenship and public space.” Sexualities, $4(1), 51-71$.

Huizinga, J. (1949). Homo ludens (Vol. 3).

Huizinga, Johan (1976) "Nature and Significance of Play as a Cultural Phenomenon" in Ritual, Play, and Performance edited by Richard Schechner and Mady Schuman 46-66

Huizinga, J. (2004). "Nature and significance of play as a cultural phenomenon." The performance studies reader, 117-120.

Hurley, M., \& Prestage, G. (2009). "Intensive sex partying amongst gay men in Sydney." Culture, Health \& Sexuality, 11(6), 597-610.

Hutzler, K., Giuliano, T., Herselman, J., \& Johnson, S. (2015). “Three's a crowd: public awareness and (mis)perceptions of polyamory." Psychology \& Sexuality, 1-19.

Hutchinson, G., \& Hutchinson, G. B. (1995). The Harlem Renaissance in black and white. Harvard University Press.

Israel, T. (2002). I. "Studying sexuality: Strategies for surviving stigma." Feminism \& Psychology, 12(2), 256-260.

Johnson, A. (2015). "Digital Media, Sex, and Performance: An Introduction." Liminalities, 11(1), 1 .

Johnston, L. (2015). "Sexuality and Space." International Encyclopedia Of The Social \& Behavioral Sciences, 808-812.

Johnston, L. (2016). "Gender and sexuality I: Genderqueer geographies?" Progress in Human Geography, 40(5), 668-678.

Johnston, L \& Longhurst, R (2009). Space, place and sex: Geographies of sexualities. Rowman and Littlefield, MD. 
Jones, O. (2011). Geography, memory and non-representational geographies. Geography Compass, 5(12), 875-885.

Joshi, S., McCutcheon, P., \& Sweet, E. L. (2015). Visceral Geographies of Whiteness and Invisible Microaggressions. ACME: An international e-journal for critical geographies, 14(1).

Kaplan, M. (2014) Sex-Positive Law. SSRN Electronic Journal. http://dx.doi.org/10.2139/ssrn.2308955

Karl, I. (2007). "On-/Offline: Gender, Sexuality, and the Techno-Polities of Everyday Life". Queer online: Media technology \& sexuality, 40, 45.

Kaspar, H., \& Landolt, S. (2014). "Flirting in the field: shifting positionalities and power relations in innocuous sexualisations of research encounters." Gender, Place \& Culture, 23(1), 107-119.

Keeling, K. (2019). Queer Times, Black Futures. NYU Press.

Keidan, L. (2015). "The 'underground' arts scene is now just a click away from the easily offended." The Guardian.

Kempadoo, K. (1998). Introduction: Globalizing sex workers rights.

Kenyon, S., Lyons, G., \& Rafferty, J. (2002). "Transport and social exclusion: investigating the possibility of promoting inclusion through virtual mobility." Journal of Transport Geography, 10(3), 207-219.

Klesse, C. (2014). "Polyamory: Intimate practice, identity or sexual orientation?" Sexualities, 17(1-2), 81-99.

Kobayashi, A. (1994). Coloring the field: Gender, "race," and the politics of fieldwork. The professional geographer, 46(1), 73-80.

Knopp, L. (1999). "Out in academia: the queer politics of one geographer's sexualisation.” Journal of Geography in Higher Education, 23(1), 116-123.

Kohl, E., \& McCutcheon, P. (2014). "Kitchen table reflexivity: negotiating positionality through everyday talk." Gender, Place \& Culture, 22(6), 747-763.

Laliberté, N., \& Schurr, C. (2015). "Introduction: The stickiness of emotions in the field: complicating feminist methodologies." Gender, Place \& Culture, 23(1), 72-78.

Lange, P. G. (2007). "Publicly private and privately public: Social networking on YouTube." Journal of computer-Mediated communication, 13(1), 361-380. 
Larvie, S. P. (1999). "Queerness and the specter of Brazilian national ruin.” GLQ: $A$ Journal of Lesbian and Gay Studies, 5(4), 527-557.

Lawson, V. (2007). "Geographies of care and responsibility." Annals of the Association of American Geographers, 97(1), 1-11.

Lawson, V. (2009). "Instead of radical geography, how about caring geography?" Antipode, 41(1), 210-213.

Leisenring, A. (2012). "The Line and Asking For It: The Ethics and Erotics of Sexual Consent." Teaching Sociology, 40(1), 74-77.

Lenius, S. (2011). “A Reflection on "Bisexuals and BDSM: Bisexual People in a Pansexual Community"-Ten Years Later (and a Preview of the Next Sexual Revolution)." Journal Of Bisexuality, 11(4), 420-425.

Levy, G. (2018) In Pursuit of Happiness: Black at Burning Man

Lidner, C., \& Hussey, A. (2016). "Concepts and Practices of the Underground.” In ParisAmsterdam underground: essays on cultural resistance, subversion, and diversion (1st ed., pp. 13-19). Amsterdam: Amsterdam University Press.

Liepe-Levinson, K. (2002). Strip show: Performances of gender and desire. Psychology Press.

Longhurst, R., Ho, E., \& Johnston, L. (2008). 'Using 'the body' as an 'instrument of research'." Area, 40(2), 208-217.

Madge, C. (2007). "Developing a geographers' agenda for online research ethics." Progress in human geography, 31(5), 654-674.

Marino, P. (2008). "The Ethics of Sexual Objectification: Autonomy and Consent." Inquiry, 51(4), 345-364.

Martin, J. (1996). "Structuring the sexual revolution." Theory And Society, 25(1), 105151.

Martin, Nina K. (2007). "Porn Empowerment: Negotiating Sex Work and Third Wave Feminism." Atlantis: Critical Studies in Gender, Culture \& Social Justice 31 (2): 31-41.

Massey, D. (2004). “Geographies of responsibility.” Geografiska Annaler: Series B, Human Geography, 86(1), 5-18.

Massey, D. (2010). A global sense of place (pp. pp-232). Aughty. org. 
Mercer, J. (2018). Sex positivity and the persistence of shame. Sexualities, 1363460718770443.

Meyer, D. S., \& Whittier, N. (1994). Social movement spillover. Social problems, 41(2), 277-298.

McCracken, J. (2013). Street sex workers' discourse: Realizing material change through agential choice. Routledge.

McDowell, L. (1992). "Doing gender: feminism, feminists and research methods in human geography." Transactions of the institute of British Geographers, 399-416.

McGlotten, S. (2013). "A brief and improper geography of queerspaces and sexpublics in Austin, Texas." Gender, Place \& Culture, 21(4), 471-488.

McLean, K. (2011). "Bisexuality and Nonmonogamy: A Reflection.” Journal of Bisexuality, 11(4), 513-517.

Megatron, S. (2015). "The Health Benefits of BDSM." About Health. Retrieved from http://sexualhealth.about.com/od/sextherapy/ss/The-Health-Benefits-of-

BDSM.htm\#showall

Meunier, E. (2014). "No attitude, no standing around: the organization of social and sexual interaction at a gay male private sex party in New York city." Archives of sexual behavior, 43(4), 685-695.

Mimiaga, M. J., Reisner, S. L., Bland, S., Cranston, K., Isenberg, D., Driscoll, M. A., ... \& Mayer, K. H. (2010). "“'It's a quick way to get what you want”: A formative exploration of HIV risk among urban Massachusetts men who have sex with men who attend sex parties." AIDS patient care and STDs, 24(10), 659-674.

Mimiaga, M. J., Reisner, S. L., Bland, S. E., Driscoll, M. A., Cranston, K., Isenberg, D., ... \& Mayer, K. H. (2011). "Sex parties among urban MSM: an emerging culture and HIV risk environment." AIDS and Behavior, 15(2), 305-318.

Mitchell, K. (2007). “Geographies of Identity: The Intimate Cosmopolitan.” Progress in Human Geography 31 (5): 706-20.

Mol, A. (2002). The body multiple: Ontology in medical practice. Duke University Press.

Moors, A., Conley, T., Edelstein, R., \& Chopik, W. (2014). “Attached to monogamy? Avoidance predicts willingness to engage (but not actual engagement) in consensual nonmonogamy.” Journal Of Social And Personal Relationships, 32(2), 222-240. 
Morrison, C., Johnston, L., \& Longhurst, R. (2012). "Critical geographies of love as spatial, relational and political.” Progress In Human Geography, 37(4), 505-521.

Morrow, O., Hawkins, R., \& Kern, L. (2014). "Feminist research in online spaces." Gender, Place \& Culture, 22(4), 526-543.

Moss, P., \& Dyck, I. (1999). "Body, corporeal space, and legitimating chronic illness: women diagnosed with ME." Antipode, 31(4), 372-397.

Moss, P., Al-Hindi, K. F., \& Kawabata, H. (2002). Feminist geography in practice: Research and methods. Wiley-Blackwell.

Mountz, A., Bonds, A., Mansfield, B., Loyd, J., Hyndman, J., Walton-Roberts, M., ... \& Curran, W. (2015). "For slow scholarship: A feminist politics of resistance through collective action in the neoliberal university." ACME: an international E-journal for critical geographies, 14(4).

Mumford, K. J. (1997). Interzones: Black/White Sex Districts in Chicago and New York in the Early Twentieth Century. Columbia University Press. Nagel, J. (2003). Race, ethnicity, and sexuality: Intimate intersections, forbidden frontiers (p. xii308). New York: Oxford University Press.

Nash, Catherine J. "Trans geographies, embodiment and experience." Gender, Place \& Culture 17, no. 5 (2010): 579-595.

Nash, C. (2003). Cultural geography: anti-racist geographies. Progress in Human Geography, 27(5), 637-648.

Nash, Jennifer. (2006). "Bearing Witness to Ghosts: Notes on Theorizing Pornography, Race, and Law.” Wisconsin Women's Law Journal 21: 47-72.

Nash, Jennifer. (2008). "Strange Bedfellows: Black Feminism and Anti-Pornography Feminism." Social Text 97: 51-76.

Neely, S. (2011). Making Bodies Visible. Transgression 2.0: Media, Culture, and the Politics of a Digital Age, 101.

Newmahr, S. (2008). "Becoming a Sadomasochist: Integrating Self and Other in Ethnographic Analysis.” Journal Of Contemporary Ethnography, 37(5), 619-643.

Nicholls, W.J. (2007). "The Geographies of Social Movements." Geography Compass $1 / 3,607-622$.

Nicholls, W.J. (2009). "Place, networks, space: theorizing the geographies of social movements" Transactions of the Institute of British Geographers. NS 34: 78-93. 
Norbeck, E. (1974). “The anthropological study of human play.” Rice Institute PamphletRice University Studies, 60(3).

O'Shea, C. D. (2007). "Before Stonewall: Activists for Gay and Lesbian Rights in Historical Context." Archives of Sexual Behavior, 36(2), 329-330.

Olund, Eric. (2010). “'Disreputable Life': Race, Sex, and Intimacy.” Environment and Planning D: Society and Space 28 (1): 142-57.

Oswin, N. (2008). "Critical Geographies and the Uses of Sexuality: Deconstructing Queer Space.” Progress in Human Geography 32 (1): 89-103.

Paasonen, S., Nikunen, K., \& Saarenmaa, L. (2007). "Pornification and the Education of Desire." Pornification: Sex and sexuality in media culture, 1-20.

Parker, R., \& Aggleton, P. (2003). HIV and AIDS-related stigma and discrimination: a conceptual framework and implications for action. Social science \& medicine, 57(1), 1324.

Phillips, David; Kate O'Riordan, David J. Phillips, Eds. (2007). Queer Online: Media Technology \& Sexuality. Peter Lang.

Pile, S. (2010). "Emotions and affect in recent human geography." Transactions Of The Institute Of British Geographers, 35(1), 5-20.

Pineau, L. (1989) “'Date Rape: A Feminist Analysis'.” Law and Philosophy 8: 217-43.

Plummer, K. (2001). "The square of intimate citizenship: Some preliminary proposals." Citizenship studies, 5(3), 237-253.

Plummer, K. (2003). Intimate citizenship. Private decisions and public dialogues.

Plummer, K. (2011). Intimate citizenship: Private decisions and public dialogues. University of Washington Press.

Potts, M. (2016). "Polyamory as a Rejection of Capitalism.” Morgan Potts. Retrieved from http://morganpotts.com/2016/polyamory-as-a-rejection-of-capitalism/

Povinelli, E. (2002). "Notes on Gridlock: Genealogy, Intimacy, Sexuality.” Public Culture, 14(1), 215-238.

Povinelli, E; Chauncy, G. (1999) "Thinking Sexuality Transnationally: An Introduction" GLQ: A Journal of Lesbian and Gay Studies 5:4 pp. 439-450. 
Pratt, A. C. (2008). Creative cities: the cultural industries and the creative class. Geografiska annaler: series B, human geography, 90(2), 107-117.

Price, K. (2010). "What is reproductive justice?: How women of color activists are redefining the pro-choice paradigm." Meridians: feminism, race, transnationalism, 10(2), 42-65.

Price-Glynn, Kim. (2010). Strip Club: Gender, Power, and Sex Work. NYU Press.

Przybylinski, S. (2014). "Finding meaning in alternative spaces.” City, 18(1), 73-77.

Puar, J. K., Rushbrook, D., \& Schein, L. (2003). "Sexuality and space: queering geographies of globalization." Environment and Planning D: Society and Space, 21(4), 383-387.

Queen, C., \& Comella, L. (2008). "The Necessary Revolution: Sex-Positive Feminism in the Post-Barnard Era.” The Communication Review, 11(3), 274-291.

Rahier, J. M. (2013). Kings for Three Days: The Play of Race and Gender in an AfroEcuadorian Festival. University of Illinois Press.

Reiss, Ira. (1990) An End to Shame: Shaping our Next Sexual Revolution. Prometheus Books, New York.

Richardson, D. (1998). “Sexuality and citizenship.” Sociology, 32(1), 83-100.

Richardson, D. (2000). "Constructing sexual citizenship: theorizing sexual rights.” Critical social policy, 20(1), 105-135.

Richardson, D. (2018). Sexuality and citizenship. Sexualities, 1363460718770449.

Robinson, M. (2013). "Polyamory and Monogamy as Strategic Identities." Journal Of Bisexuality, 13(1), 21-38.

Rofel, L. (1999). "Qualities of desire: imagining gay identities in China." GLQ: A Journal of Lesbian and Gay Studies, 5(4), 451-474.

Rose, G. (1997). "Situating knowledges: positionality, reflexivities and other tactics." Progress in human geography, 21(3), 305-320.

Ross, M. (2005). "Typing, doing, and being: Sexuality and the internet." Journal Of Sex Research, 42(4), 342-352.

Rubin, Lillian. (1990) Erotic Wars: What Happened to the Sexual Revolution? Farrar, Straus and Giroux, New York. 
Sanchez, M. E. (2019). Queer Faith: Reading Promiscuity and Race in the Secular Love Tradition. NYU Press.

Schurr, C., \& Abdo, K. (2015). "Rethinking the place of emotions in the field through social laboratories." "Gender, Place \& Culture, 23(1), 120-133.

Seidman, S. (1995). "Deconstructing queer theory or the under-theorization of the social and the ethical." Social postmodernism: Beyond identity politics, 116-141.

Sheff, E., \& Hammers, C. (2011). "The privilege of perversities: race, class and education among polyamorists and kinksters.” Psychology \& Sexuality, 2(3), 198-223.

Sigusch, Volkmar (1998). “The Neosexual Revolution” Archives of Sexual Behavior, Vol. 27, No. 4, pp. 331-359.

Smith, C. (2010). "Pornographication: A discourse for all seasons." International Journal of Media \& Cultural Politics, 6(1), 103-108.

Smith, S. (2014). "Intimacy and angst in the field." Gender, Place \& Culture, 23(1), 134146.

Smith, C., \& Attwood, F. (2014). “Anti/pro/critical porn studies.” Porn Studies, 1(1-2), 723.

Solomon, T. M., Halkitis, P. N., Moeller, R. M., Siconolfi, D. E., Kiang, M. V., \& Barton, S. C. (2011). "Sex parties among young gay, bisexual, and other men who have sex with men in New York City: Attendance and behavior." Journal of Urban Health, 88(6), 1063-1075.

St John, G. (2018). Civilised Tribalism: Burning Man, Event-Tribes and Maker Culture. Cultural Sociology, 12(1), 3-21.

Staeheli, L. A., Kofman, E., \& Peake, L. (2004). Mapping women, making politics:

Feminist perspectives on political geography. Psychology Press.

Steele, V. (2015). “Fetish Fashion.” Lovetoknow.com.

Stewart, M. R. (2013). "Getting Freaky": Perversion and Promiscuity Within Alternative Relationships and Sexual Modalities Among People Of Color.

Taormino, Tristan. (2013). The Feminist Porn Book: The Politics of Producing Pleasure. New York: Feminist Press at the City University of New York. 
Tauqir, Tamsila, Jennifer Petzen, Jin Haritaworn, Sokari Ekine, Sarah Bracke, Sarah Lamble, Suhraiya Jivraj, and Stacy Douglas (2011). Queer anti-racist activism and strategies of critique: A roundtable discussion. Feminist legal studies, 19(2), 169.

Taylor, J. (2011). "The intimate insider: negotiating the ethics of friendship when doing insider research." Qualitative Research, 11(1), 3-22.

Thien, D. (2005). "After or beyond feeling? A consideration of affect and emotion in geography." Area, 37(4), 450-454.

Trahan, H. (2014). Relationship Literacy And Polyamory: A Queer Approach (PhD Dissertation). Bowling Green State University.

Trumbach, Randolph (December 2014). "Review of The Origins of Sex: A History of the First Sexual Revolution.” The Journal of Modern History, Vol. 86, No. 4, pp. 868-869.

Turner, V. (1974). "Liminal to liminoid, in play, flow, and ritual: an essay in comparative symbology." Rice Institute Pamphlet-Rice University Studies, 60(3).

Turner, V. (1979). "Frame, flow and reflection: Ritual and drama as public liminality." Japanese Journal of Religious Studies, 465-499.

Turner, V. (1982). From Ritual to Performance: The Human Seriousness of Play. New York: Performing Arts Journal Publications.

Turner, V. W., \& Schechner, R. (1988). The anthropology of performance.

Tweedy, A. (2011). "Polyamory as a Sexual Orientation." University Of Cincinnati Law Review, 79(4), 1461-1515.

Twine, F. W., \& Gardener, B. (2013). Displacing Place Identity: Introducing an Analytics of Participation. In Geographies of Privilege (pp. 65-92). Routledge.

Valentine, G. (2008). "The Ties That Bind: Towards Geographies of Intimacy." Geography Compass, 2(6), 2097-2110.

Van Anders, S. M. (2015). "Beyond sexual orientation: Integrating gender/sex and diverse sexualities via sexual configurations theory." Archives of Sexual Behavior, 44(5), 1177-1213.

Vogel, S. (2009). The scene of Harlem cabaret: Race, sexuality, performance. University of Chicago Press. 
Vogel, S. (2013). "The Sensuous Harlem Renaissance" Conference paper given at Queer Urbanity: The Second Annual Black Queer Sexuality Studies Graduate Student Conference, Princeton University.

Warner, M. (2000). The trouble with normal: Sex, politics, and the ethics of queer life. Harvard University Press.

Watts, E. K. (2007). Queer Harlem: Exploring the Rhetorical Limits of a Black Gay 'Utopia.'. Queering public address: Sexualities in American historical discourse, 174-94.

Weeks, J. (1996). “The idea of a sexual community.” Soundings, 2, 71-83.

Weeks, J. (1998). “The sexual citizen.” Theory, culture \& society, 15(3), 35-52.

Weiss, M. D. (2006). "Mainstreaming kink: The politics of BDSM representation in US popular media." Journal of Homosexuality, 50(2-3), 103-132.

Weiss, M. D. (2008). Gay shame and BDSM pride: Neoliberalism, privacy, and sexual politics. Radical History Review, (100).

Weiss, Margot (2011) Techniques of Pleasure: BDSM and the Circuits of Sexuality. Duke University Press

White, V. (2004). “A Humanist Looks at Polyamory." The Humanist, November/December 2004, 17-20.

Whittaker, Polly. (2014). Polly: Sex Culture Revolutionary. Moral Minority, Inc.

Wilkinson, E. (2009). "The emotions least relevant to politics? Queering autonomous activism." Emotion, Space and Society, 2(1), 36-43.

Wilkinson, E. (2010). “What's Queer About Non Monogamy Now?” In M. Barker \& D. Langrdridge, Understanding Non-Monogamies (1st ed.). Routledge: London New York.

Wilkinson, E. (2011). “'Extreme pornography' and the contested spaces of virtual citizenship.” Social \& Cultural Geography, 12(5), 493-508.

Williams, L. (Ed.). (2004). Porn studies. Duke University Press.

Wilson, J. F. (2010). Bulldaggers, pansies, and chocolate babies: performance, race, and sexuality in the Harlem Renaissance. University of Michigan Press.

Wolf, D. L. (1993). "Introduction: Feminist dilemmas in fieldwork." Frontiers: A Journal of Women Studies, 1-8. 
Wolfe, Leanna. (2008). "On kittens and the very invented culture of polyamory." Electronic Journal of Human Sexuality 11:30.

Woodward, K., \& Lea, J. (2010). Geographies of affect (pp. 154-175). S. Smith, R. Pain, \& S. A. Marston (Eds.). London: Sage.

Woodyer, T. (2012). "Ludic geographies: Not merely child's play." Geography Compass, 6(6), 313-326.

Wouters, C. (1998). Balancing sex and love since the 1960s sexual revolution. Theory, culture \& society, 15(3-4), 187-214.

Wouters, C. (2010). "Sexualization: Have sexualization processes changed direction?" Sexualities, 13(6), 723-741.

Wosick, K. R. (2011). Swinging in America: Love, Sex, and Marriage in the 21st Century. 
VITA

\author{
CHRISTINA N. BAZZARONI \\ 2030 NW Flanders Street, 207 \\ Portland, OR 97209 \\ cbazzaroni@gmail.com
}

\title{
Education
}

Areas Of Specialization and Teaching Interests: African \& African Diaspora studies; Critical Feminist Geographies and Queer theory; Cultural Anthropology; Cultural and Human Geography; Ethnography; Geographies of Sexuality; Interdisciplinary studies; Methods in Geography; Qualitative methods; Women's and Gender studies

2011 - Present: Florida International University, Miami

PhD Global \& Sociocultural Studies, Geography

Dissertation: Transnational Sex-Positive Play Parties: The Sexual Politics of Care for Community-Making at a Kinky Salon (Chair: Dr. Jean Rahier; Co-Chair: Dr. Caroline Faria)

2009 - Present2011: Florida International University, Miami

M.A. African \& African Diaspora Studies

1994 - 1998: University of California, Davis

B.A. Psychology

B.A. Sociology with emphasis in Law and Society

Minor: English Literature

(2018) Dissertation Year Fellow, Florida International University. Total funding awarded: $\$ 16,600$, tuition waiver, health insurance.

\section{$\underline{\text { Publications and Conference Presentations }}$}

Meszaros, J., \& Bazzaroni, C. (2014). From taboo to tourist industry: The construction of interracial intimacies between Black men and White women in colonial and contemporary times. Sociology Compass, 8(11):1256-1268.

(2018) Association of American Geographers, New Orleans. Paper presentation: "Spacemaking Through the Shifting Places of Sex-Positive Community."

(2017) European Geographies of Sexualities Conference, Barcelona Spain. Paper presentation: "Feminist Care Ethics and Sexuality: Connecting Through Play in a Sex Positive Community." 
(2016) Association of American Geographers, San Francisco. Paper presentation: "Sex Positive Culture Creation: Kinky Salon and the Sex Culture Revolution."

(2016) Association of American Geographers, San Francicso, Sexuality and Space Specialty Group Pre-Conference. Paper presentation: "Sexuality Research and Self Care."

(2015) European Geographies of Sexualities Conference, Rome, Italy. Paper presentation: "This Sex Culture Revolution will not be Televised: Understanding the Technological Mobilization of Kinky Salon."

(2014) National Women's Studies Association Annual Conference, San Juan, Puerto Rico. Paper presentation: "Sex, Performance, and the City: Exploring New York City's Kinky Underground."

(2014) Association of American Geographers Annual Meeting/Conference, Tampa, FL. Paper presentation: "Behind Closed Doors: Sex, Race, and Performance in the NYC Erotic Underground" - Preliminary Findings.

(2012) Toyin Falola Annual Conference on Africa and the African Diaspora, Aja, Lagos, Nigeria. Paper presentation: "Dangerous Zones of Intimacy: Transgressive White Female Desire, or Fetishization of the Black Male Body?"

(2012) National Council of Black Studies Annual Conference, Atlanta, GA. Paper presentation: "Spirituality in Hip Hop: A Proposed Case Study."

(2012) Association of American Geographers Annual Meeting/ Conference, New York, NY. Paper presentation: "Spirituality in Hip Hop." 